\title{
On logarithmic nonabelian Hodge theory of higher level in characteristic $p$
}

\author{
SACHIO OHKAWA (*)
}

ABstraCt - Given a natural number $m$ and a log smooth integral morphism $X \rightarrow S$ of fine $\log$ schemes of characteristic $p>0$ with a lifting of its Frobenius pull-back $X^{\prime} \rightarrow S$ modulo $p^{2}$, we use indexed algebras $\mathcal{A}_{X}^{g p}, \mathcal{B}_{X / S}^{(m+1)}$ of Lorenzon-Montagnon and the sheaf $\mathcal{D}_{X / S}^{(m)}$ of log differential operators of level $m$ of Berthelot-Montagnon to construct an equivalence between the category of certain indexed $\mathcal{A}_{X}^{g p}$-modules with $\mathcal{D}_{X / S}^{(m)}$-action and the category of certain indexed $\mathcal{B}_{X / S}^{(m+1)}$-modules with Higgs field. Our result is regarded as a level $m$ version of some results of Ogus-Vologodsky and Schepler.

Mathematics SubJeCt Classification (2010). 16H05; 14F30, 32C38.

Keywords. Log geometry, Log $\mathcal{D}$-module, Higgs module, Cartier transform.

\section{Introduction}

For a projective smooth complex algebraic variety, Simpson [12] established a correspondence, which is called the Simpson correspondence nowadays, between local systems and Higgs bundles. In [10], Ogus and Vologodsky studied an analogue of the Simpson correspondence for certain integrable connections or equivalently certain $\mathcal{D}$-modules in positive characteristic. As a natural generalization of their theory, Schepler [11] studied its log version and Gros, Le Stum and Quirós [4] studied its higher level version. The aim of this article is to establish the log and higher level version of the theory of OgusVologodsky.

Let us recall the Ogus-Vologodsky's analogue of the Simpson correspondence in positive characteristic, which is called the global Cartier transform (see Theorem 2.8 of [10]). Let $X \rightarrow S$ be a smooth morphism of schemes of characteristic $p>0$. Let us denote by $X^{\prime}$ the pull-back of $X \rightarrow S$ via the absolute

(*) Indirizzo dell'A.: Sachio Ohkawa, Graduate School of Mathematical Sciences, University of Tokyo, 3-8-1 Komaba, Meguro-ku, Tokyo 153-8914, Japan.

E-mail: ohkawa@ms.u-tokyo.ac.jp 
Frobenius $F_{S}$ of $S$. Denote the relative Frobenius morphism $X \rightarrow X^{\prime}$ by $F_{X / S}$. Let $\mathcal{T}_{X^{\prime} / S}$ be the tangent bundle of $X^{\prime}$ over $S, S \mathcal{T}_{X^{\prime} / S}$ the symmetric algebra of $\mathcal{T}_{X^{\prime} / S}$ and $\mathcal{G}$ the nilpotent divided power envelope of the zero section of the cotangent bundle of $X^{\prime} / S$, so that $\mathcal{O}_{\mathcal{G}}=\hat{\Gamma} \cdot \mathcal{I}_{X^{\prime} / S}$. Assume that we are given a lifting of $X^{\prime} \rightarrow S$ modulo $p^{2}$. Then there exists an equivalence between the category of $\mathcal{O}_{X}$-modules $E$ with integrable connection $\nabla$ equipped with a horizontal $\mathcal{O}_{X^{-}}$ linear $\mathcal{G}$-Higgs field $\theta: \mathcal{O}_{\mathcal{G}} \rightarrow F_{X / S *} \mathcal{E} n d_{\mathcal{O}_{X}}(E, \nabla)$ extending the horizontal map $\psi: S \mathcal{T}_{X^{\prime} / S} \rightarrow F_{X / S *} \mathcal{E} n d_{\mathcal{O}_{X}}(E, \nabla)$ given by the $p$-curvature and the category of $\mathcal{O}_{X^{\prime}}$-modules $E^{\prime}$ equipped with an $\mathcal{O}_{X^{\prime}}$-linear $\mathcal{G}$-Higgs field $\theta: \mathcal{O}_{\mathcal{G}} \rightarrow \mathcal{E} n d_{\mathcal{O}_{X^{\prime}}}\left(E^{\prime}\right)$. There are two key technical results for the proof of the global Cartier transform. One is the fact that the sheaf $\mathcal{D}_{X / \mathcal{S}}^{(0)}$ of differential operators of level 0 on $X$ is an Azumaya algebra over its center, which is isomorphic to $S \mathcal{T}_{X^{\prime} / S}$ via the $p$-curvature map. The other is a construction of the splitting module $\check{\mathcal{K}}_{\mathcal{X} / \mathcal{S}}$ for this Azumaya algebra over the scalar extension $\mathcal{O}_{\mathcal{G}}$ of $S \cdot \mathcal{T}_{X^{\prime} / S}$. This means an isomorphism of $\mathcal{O}_{X}$-algebras $\mathcal{D}_{X / S}^{(0)} \otimes_{S} \mathcal{T}_{X^{\prime} / S} \mathcal{O}_{\mathcal{G}} \stackrel{\cong}{\Rightarrow} \mathcal{E} n d_{\mathcal{O}_{\mathcal{G}}}\left(\check{\mathcal{K}}_{\mathcal{X} / \mathcal{S}}\right)$. Then the global Cartier transform can be obtained by the Morita equivalence. Ogus-Vologodsky also constructed a splitting module over the completion $\hat{S} \mathcal{T}_{X^{\prime} / S}$ of $S \cdot \mathcal{T}_{X^{\prime} / S}$ under the assumption of an existence of a $\bmod p^{2}$ lifting of $F_{X / S}$ and got an analogous equivalence called the local Cartier transform.

As is mentioned in the first paragraph, the theory of Ogus-Vologodsky has been generalized in (at least) two directions. First, Schepler [11] extended their theory to the case of $\log$ schemes. The difficulty for this generalization is that the Azumaya nature of the sheaf $\mathcal{D}_{X / S}^{(0)}$ of the log differential operators of level 0 is no longer true in general. Schepler overcame this difficulty by using Lorenzon's theory of indexed modules and indexed algebras $\mathcal{A}_{X}^{g p}$ and $\mathcal{B}_{X / S}$ associated to a $\log$ scheme $X$ and its Frobenius pullback $X^{\prime} \rightarrow S$. Roughly speaking, $\mathcal{A}_{X}^{g p}$ and $\mathcal{B}_{X / S}$ are the suitable scalar extensions of the structure sheaf $\mathcal{O}_{X}$ and $\mathcal{O}_{X^{\prime}}$ respectively in the case of $\log$ schemes. He used the sheaf $\tilde{\mathcal{D}}_{X / S}^{(0)}:=$ $\mathcal{A}_{X}^{g p} \otimes_{\mathcal{O}_{X}} \mathcal{D}_{X / S}^{(0)}$ in place of $\mathcal{D}_{X / S}^{(0)}$ and proved the Azumaya nature of $\tilde{\mathcal{D}}_{X / S}^{(0)}$ over its center. Schepler also generalized the splitting module $\check{\mathcal{K}}_{\mathcal{X} / \mathcal{S}}$ of Ogus-Vologodsky and got the log global Cartier transform. Second, in [4], Gros, Le Stum and Quirós extended some results in [10] to the case of Berthelot's ring of differential operators of higher level [2]. They proved the Azumaya nature of the sheaf $\mathcal{D}_{X / S}^{(m)}$ of differential operators of level $m$, constructed a splitting module for $\mathcal{D}_{X / S}^{(m)}$ over $\hat{S} \cdot \mathcal{T}_{X^{\prime} / S}$ (here $X^{\prime}$ denotes the pull-back of $X \rightarrow S$ by the $(m+1)$-st iterate of the absolute Frobenius $F_{S}: S \rightarrow S$ ) under the assumption of an existence of a good lifting of the $(m+1)$-st relative Frobenius morphism $F_{X / S}$ $\bmod p^{2}$, which they call a strong lifting, and proved the local Cartier transform of higher level. They also constructed (but informally) a global splitting module by a gluing argument. But their construction is different from that of OgusVologodsky. It should be remarked here that the sheaf $\tilde{\mathcal{D}}_{X / S}^{(0)}$ used by Schepler (or more generally the sheaf $\tilde{\mathcal{D}}_{X / S}^{(m)}$ of log differential operators of higher level) was introduced by Montagnon [9]. She established there the foundations of log 
differential operators of higher level and especially obtained the log version of Berthelot's Frobenius descent by using the indexed algebras $\mathcal{A}_{X}^{g p}$ and $\mathcal{B}_{X / S}^{(m)}$, where the latter denotes the higher level version of Lorenzon's $\mathcal{B}_{X / S}$.

The purpose of this paper is to generalize Schepler's log global Cartier transform to the case of higher level by using the indexed algebras $\mathcal{A}_{X}^{g p}$ and $\mathcal{B}_{X / S}^{(m+1)}$ of Lorenzon and Montagnon. Our construction is a natural generalization of Ogus-Vologodsky and Schepler, but we also need some log differential calculus of higher level which is based on Montagnon's result. We also prove the compatibility of the log global Cartier transform with Montagnon's $\log$ Frobenius descent.

Let us describe the content of each section. We work with a log smooth morphism $X \rightarrow S$ of fine $\log$ schemes in positive characteristic. Let $F_{X / S}$ denote the $(m+1)$-st relative Frobenius $X \rightarrow X^{\prime}$. In the second section, we review the theory of indexed modules. In the third section, we construct the log version of the higher curvature map $\beta: \mathcal{T}_{X^{\prime} / S} \rightarrow F_{X / S *} \mathcal{D}_{X / S}^{(m)}$, which we call the $p^{m+1}$-curvature map, in Definition 3.10 after reviewing the theory of $\log$ differential operators of level $m$. In the fourth section, after reviewing the construction and some basic results of indexed algebras associated to the log structure, we study the Azumaya nature of $\tilde{\mathcal{D}}_{X / S}^{(m)}$. We prove that $\mathcal{B}_{X / S}^{(m+1)} \otimes_{\mathcal{O}_{X^{\prime}}} S \cdot \mathcal{T}_{X^{\prime} / S}$ is identified with the center of $\tilde{\mathcal{D}}_{X / S}^{(m)}$ via the $p^{m+1}$-curvature map (see Theorem 4.16) and $\tilde{\mathcal{D}}_{X / S}^{(m)}$ is an Azumaya algebra over $\mathcal{B}_{X / S}^{(m+1)} \otimes_{\mathcal{O}_{X^{\prime}}} S \cdot \mathcal{T}_{X^{\prime} / S}$ (see Corollary $4.2-$ ). We also prove the log Cartier descent theorem of higher level as an application (see Theorem 4.26). In the fifth section, we construct the splitting module $\check{\mathcal{K}}_{\mathcal{X} / \mathcal{S}}^{(m), \mathcal{A}}$ for $\tilde{\mathcal{D}}_{X / S}^{(m)}$ over $\mathcal{B}_{X / S}^{(m+1)} \otimes_{\mathcal{O}_{X^{\prime}}} \hat{\Gamma} \mathcal{T}_{X^{\prime} / S}$ under the assumption of an existence of a mod $p^{2}$ lifting of $X^{\prime} \rightarrow S$ (see (18)) and get the log global Cartier transform of higher level by using the indexed variant of the Morita equivalence (see Theorem 5.19). In the final section, we consider the compatibility of the log global Cartier transform with Montagnon's log Frobenius descent. Our new ingredient is to prove the behavior of the splitting module $\check{\mathcal{K}}_{\mathcal{X} / \mathcal{S}}^{(m), \mathcal{A}}$ with respect to the Frobenius descent functor of Montagnon (see Theorem 6.10). As a consequence of Theorem 6.10, we obtain the expected compatibility (see Theorem 6.8).

\section{Indexed Azumaya Algebra}

In this section, we give a review of the theory of indexed modules and indexed Azumaya algebras developed by ([5], see also [11]) which we will use to construct the log global Cartier transform of higher level. The general theory of indexed modules can be developed on a ringed topos but, for simplicity, we only consider the case of the ringed topos associated to the étale site of a scheme and its structure sheaf. Also, we try to describe several notions more concretely than those given in [5] and [11]. We fix throughout this section a scheme $X$ and an étale sheaf of abelian groups $\mathcal{I}$. 


\section{1 - Indexed module}

Let us recall some notions on indexed modules.

Definition 2.1. (1) An $\mathcal{I}$-indexed sheaf on $X$ is a sheaf of sets over $\mathcal{I}$, namely, a map of sheaves $\mathcal{F} \rightarrow \mathcal{I}$. We denote the map $\mathcal{F} \rightarrow \mathcal{I}$ by $p_{\mathcal{F}}$. An $\mathcal{I}$-indexed sheaf of abelian groups on $X$ is an $\mathcal{I}$-indexed sheaf $\mathcal{F} \rightarrow \mathcal{I}$ on $X$ equipped with an addition map $\mathcal{F} \times_{\mathcal{I}} \mathcal{F} \rightarrow \mathcal{F}$ over $\mathcal{I}$, a unit map $\mathcal{I} \rightarrow \mathcal{F}$ over $\mathcal{I}$ and an inverse map $\mathcal{F} \rightarrow \mathcal{F}$ over $\mathcal{I}$ satisfying the usual axioms of abelian groups.

(2) An $\mathcal{I}$-indexed $\mathcal{O}_{X}$-module is an $\mathcal{I}$-indexed sheaf of abelian groups equipped with a scalar multiplication map $\mathcal{O}_{X} \times \mathcal{F} \rightarrow \mathcal{F}$ over $\mathcal{I}$ satisfying the usual associativity, distributivity and unitarity conditions, where $\mathcal{O}_{X} \times \mathcal{F}$ is regarded as a sheaf over $\mathcal{I}$ via the composite $\mathcal{O}_{X} \times \mathcal{F} \rightarrow \mathcal{F} \rightarrow \mathcal{I}$.

(3) An $\mathcal{I}$-indexed $\mathcal{O}_{X}$-algebra is an $\mathcal{I}$-indexed $\mathcal{O}_{X}$-module $\mathcal{A}$ equipped with an $\mathcal{O}_{X}$-bilinear multiplication map $\pi: \mathcal{A} \times \mathcal{A} \rightarrow \mathcal{A}$ over the addition map $\mathcal{I} \times \mathcal{I} \rightarrow \mathcal{I}$ and a global section $1_{\mathcal{A}}$ of $\mathcal{A}$ over the zero section $0: e \rightarrow \mathcal{I}$ satisfying the usual associativity and unitarity conditions. We say an $\mathcal{I}$-indexed $\mathcal{O}_{X}$-algebra $\mathcal{A}$ is commutative if the multiplication map $\pi$ satisfies $\pi \circ \sigma=\pi$ where $\sigma$ is the isomorphism $\mathcal{A} \times \mathcal{A} \rightarrow \mathcal{A} \times \mathcal{A}$ defined by $(a, b) \mapsto(b, a)$.

(4) For an $\mathcal{I}$-indexed $\mathcal{O}_{X}$-algebra $\mathcal{A}$, an $\mathcal{I}$-indexed $\mathcal{A}$-algebra is an $\mathcal{I}$-indexed $\mathcal{O}_{X}$-algebra $\mathcal{B}$ equipped with a morphism $\mathcal{A} \rightarrow \mathcal{B}$ of $\mathcal{I}$-indexed $\mathcal{O}_{X}$-algebras.

REMark 2.2. Let $\mathcal{A}$ be an $\mathcal{I}$-indexed sheaf on $X$. For an étale open $U$ of $X$ and a section $i \in \mathcal{I}(U)$, we denote by $\mathcal{A}_{i}$ the pullback $h_{U} \times_{\mathcal{I}} \mathcal{A}$ where $h_{U}$ is an étale sheaf on $X$ represented by $U$ and $h_{U} \rightarrow \mathcal{I}$ is the section $i$. We call $\mathcal{A}_{i}$ the fiber of $\mathcal{A} \rightarrow \mathcal{I}$ at $i \in \mathcal{I}(U)$. Note that $\mathcal{A}_{i}$ is naturally considered as an étale sheaf on $U$, and moreover, if $\mathcal{A}$ is an $\mathcal{I}$-indexed $\mathcal{O}_{X}$-module, then $\mathcal{A}_{i}$ has an $\mathcal{O}_{U}$-module structure naturally induced by the $\mathcal{I}$-indexed $\mathcal{O}_{X}$-module structure on $\mathcal{A}$. If $\mathcal{A}$ is an $\mathcal{I}$-indexed $\mathcal{O}_{X}$-algebra, the multiplication map $\pi$ of $\mathcal{A}$ is equivalent to the following data: for each étale open $U$ of $X$ and sections $i, j \in \mathcal{I}(U)$, a morphism of $\mathcal{O}_{U}$-modules $\pi_{i j}: \mathcal{A}_{i} \otimes_{\mathcal{O}_{U}} \mathcal{A}_{j} \rightarrow \mathcal{A}_{i+j}$ functorial with respect to $i, j$ satisfying the obvious conditions of associativity and unitarity.

Now we recall the definition of $\mathcal{J}$-indexed $\mathcal{A}$-modules.

DeFinition 2.3. Let $\mathcal{A}$ be an $\mathcal{I}$-indexed $\mathcal{O}_{X}$-algebra. Let $\mathcal{J}$ be an étale sheaf of $\mathcal{I}$-sets, that is, an étale sheaf of sets on $X$ equipped with an $\mathcal{I}$-action map $\mathcal{I} \times \mathcal{J} \rightarrow \mathcal{J} ;(i, j) \mapsto i+j$. A $\mathcal{J}$-indexed left $\mathcal{A}$-module is a $\mathcal{J}$-indexed $\mathcal{O}_{X}$-module $\mathcal{E}$ equipped with an $\mathcal{O}_{X}$-bilinear map $\rho: \mathcal{A} \times \mathcal{E} \rightarrow \mathcal{E}$ over the $\mathcal{I}$-action map $\mathcal{I} \times \mathcal{J} \rightarrow \mathcal{J}$ satisfying the usual associativity and unitarity conditions. We can similarly define the notion of $\mathcal{J}$-indexed right $\mathcal{A}$-module.

REMARK 2.4. Let $\mathcal{A}$ be an $\mathcal{I}$-indexed $\mathcal{O}_{X}$-algebra and $\mathcal{J}$ be an étale sheaf of $\mathcal{I}$ sets on $X$. Let $\mathcal{E}$ be a $\mathcal{J}$-indexed left $\mathcal{A}$-module. Then the structure morphism 
$\rho: \mathcal{A} \times \mathcal{E} \rightarrow \mathcal{E}$ over $\mathcal{I} \times \mathcal{J} \rightarrow \mathcal{J}$ is equivalent to the following data: for each étale open $U$ of $X$, and each section $(i, j) \in \mathcal{I} \times \mathcal{J}$, a morphism of $\mathcal{O}_{U}$-modules $\rho_{i j}: \mathcal{A}_{i} \otimes_{\mathcal{O}_{U}} \mathcal{E}_{j} \rightarrow \mathcal{E}_{i+j} ; a \otimes e \mapsto a e$ functorial with respect to $i, j$ satisfying the obvious conditions of associativity and unitarity.

Next we recall the definition of tensor products and internal hom objects as an indexed module.

Definition 2.5. Let $\mathcal{A}$ be an $\mathcal{I}$-indexed $\mathcal{O}_{X}$-algebra and $\mathcal{J}, \mathcal{K}$ be étale sheaves of $\mathcal{I}$-sets on $X$.

(1) Let $\mathcal{E}$ be a $\mathcal{J}$-indexed right $\mathcal{A}$-module and $\mathcal{F}$ a $\mathcal{K}$-indexed left $\mathcal{A}$-module. Let $\mathcal{J} \otimes_{\mathcal{I}} \mathcal{K}$ be the $\mathcal{I}$-set $\mathcal{J} \times \mathcal{K} / \sim$, where $\sim$ is the equivalence relation generated by the relation $(i+j, k) \sim(j, i+k)$ for $i \in \mathcal{I}, j \in \mathcal{J}, k \in \mathcal{K}$. Then we define a $\mathcal{J} \otimes_{\mathcal{I}} \mathcal{K}$ indexed sheaf of abelian groups $\mathcal{E} \otimes_{\mathcal{A}} \mathcal{F}$ (the tensor product of $\mathcal{E}$ and $\mathcal{F}$ ) as the object representing the functor which sends $\mathcal{J} \otimes_{\mathcal{I}} \mathcal{K}$-indexed sheaf of abelian groups $\mathcal{M}$ to the set of biadditive $\mathcal{A}$-balanced morphisms $\mathcal{E} \times \mathcal{F} \rightarrow \mathcal{M}$ over the natural projection $\mathcal{J} \times \mathcal{K} \rightarrow \mathcal{J} \otimes_{\mathcal{I}} \mathcal{K}$. Concretely this is the étale sheaf on $X$ associated to the presheaf

$$
U \longmapsto \bigsqcup_{l \in \mathcal{J} \otimes_{\mathcal{I}} \mathcal{K}(U)}\left(\bigoplus_{(j, k)=l} \mathcal{E}_{j}(U) \otimes_{\mathcal{O}_{X}(U)} \mathcal{F}_{k}(U)\right) / R
$$

endowed with the natural projection to $\mathcal{J} \otimes_{\mathcal{I}} \mathcal{K}$, where $R$ is the $\mathcal{O}_{X}(U)$-submodule generated by

$\left\{x a \otimes y-x \otimes a y \mid x \in \mathcal{E}(U), \begin{array}{l}y \in \mathcal{F}(U) \text { and } a \in \mathcal{A}(U) \text { satisfying } \\ \left(p_{\mathcal{E}}(x)+p_{\mathcal{A}}(a), p_{\mathcal{F}}(y)\right)=l\end{array}\right\}$. When $\mathcal{A}$ is commutative, then $\mathcal{E} \otimes_{\mathcal{A}} \mathcal{F}$ naturally forms a $\mathcal{J} \otimes_{\mathcal{I}} \mathcal{K}$-indexed $\mathcal{A}$-module.

(2) For a $\mathcal{K}$-indexed left $\mathcal{A}$-module $\mathcal{F}$ and $\varphi \in \operatorname{Hom}_{\mathcal{I}}(\mathcal{J}, \mathcal{K})$, we define the $\mathcal{J}$ indexed $\mathcal{A}$-module $\mathcal{F}(\varphi)$ by the étale sheaf $\mathcal{F} \times \times_{\mathcal{K}, \varphi} \mathcal{J}$ with the second projection and the $\mathcal{A}$-action via the action on $\mathcal{F}$.

(3) Let $\mathcal{E}$ be a $\mathcal{J}$-indexed left $\mathcal{A}$-module and $\mathcal{F}$ a $\mathcal{K}$-indexed left $\mathcal{A}$-module. We define the internal hom object of $\mathcal{E}$ and $\mathcal{F}$, which we denote by $\mathcal{H o m}_{\mathcal{A}}(\mathcal{E}, \mathcal{F})$, as the étale sheaf on $X$

$$
U \longmapsto \bigsqcup_{\varphi \in \mathcal{H} m_{\mathcal{I}}(\mathcal{J}, \mathcal{K})(U)} \operatorname{Hom}_{\mathcal{A}}\left(\left.\mathcal{E}\right|_{U},\left.\mathcal{F}\right|_{U}(\varphi)\right)
$$

endowed with the natural projection to $\mathcal{H o m}_{\mathcal{I}}(\mathcal{J}, \mathcal{K})$, where $\operatorname{Hom}_{\mathcal{A}}$ denotes the set of homomorphism of $\mathcal{J}$-indexed $\mathcal{A}$-modules. When $\mathcal{A}$ is commutative, then $\mathcal{H o m}_{\mathcal{A}}(\mathcal{E}, \mathcal{F})$ naturally forms a $\mathcal{H o m}_{\mathcal{I}}(\mathcal{J}, \mathcal{K})$-indexed $\mathcal{A}$-module. Also, we denote $\mathcal{H o m}_{\mathcal{A}}(\mathcal{E}, \mathcal{E})$ simply by $\mathcal{E} n d_{\mathcal{A}}(\mathcal{E})$.

Finally we recall the local freeness and faithful flatness as an $\mathcal{I}$-indexed $\mathcal{A}$ module. 
Definition 2.6. Let $\mathcal{A}$ be an $\mathcal{I}$-indexed $\mathcal{O}_{X}$-algebra and let $\mathcal{B}$ be a $\mathcal{J}$-indexed $\mathcal{A}$-algebra.

(1) We say that an $\mathcal{I}$-indexed $\mathcal{A}$-module $\mathcal{E}$ is locally free of rank $k$ if étale locally on $X$ there exist sections $n_{1}, \ldots, n_{k}$ of $\mathcal{I}=\mathcal{H o m}_{\mathcal{I}}(\mathcal{I}, \mathcal{I})$ such that $\mathcal{E}$ is isomorphic to $\bigoplus_{i=1}^{k} \mathcal{A}\left(n_{i}\right)$, where $\mathcal{A}\left(n_{i}\right)$ are as in Definition $2.5(2)$.

(2) We say that $\mathcal{B}$ is faithfully flat over $\mathcal{A}$ if the functor $\mathcal{E} \mapsto \mathcal{E} \otimes_{\mathcal{A}} \mathcal{B}$ is exact and faithful.

\section{2 - Indexed Azumaya algebra}

The following proposition due to Schepler (see [11]) is an index version of the Morita equivalence.

Proposition 2.7. Let $\mathcal{A}$ be a commutative $\mathcal{I}$-indexed $\mathcal{O}_{X}$-algebra. Let $\mathcal{J}$ be an étale sheaf of $\mathcal{I}$-sets on $X$ and $M$ be a locally free $\mathcal{I}$-indexed $\mathcal{A}$-module of finite rank. We denote $\mathcal{E n d}_{\mathcal{A}}(M)$ by $\mathcal{E}$ which is an $\mathcal{I}$-indexed $\mathcal{O}_{X}$-algebra in natural way. Then the functor $E \longmapsto M \otimes_{\mathcal{A}} E$ is an equivalence of categories between the category of $\mathcal{J}$-indexed $\mathcal{A}$-modules and the category of $\mathcal{J}$-indexed left $\mathcal{E}$-modules.

Proof. The quasi-inverse of $E \longmapsto M \otimes_{\mathcal{A}} E$ is given by $F \longmapsto \mathcal{H} \operatorname{Hom}_{\mathcal{A}}(M, F)$. For more details, see Theorem 2.2 of [11].

Now let us recall the notion on indexed Azumaya algebra.

Definition 2.8. Let $\mathcal{A}$ be a commutative $\mathcal{I}$-indexed $\mathcal{O}_{X}$-algebra and $\mathcal{E}$ an $\mathcal{I}$ indexed $\mathcal{A}$-algebra. Then, for a commutative $\mathcal{I}$-indexed $\mathcal{A}$-algebra $\mathcal{B}, \mathcal{E}$ splits over $\mathcal{B}$ with splitting module $M$ if there exists an $\mathcal{I}$-indexed locally free $\mathcal{B}$-module $M$ of finite rank such that $\mathcal{E} \otimes_{\mathcal{A}} \mathcal{B} \cong \mathcal{E} n d_{\mathcal{B}}(M)$. $\mathcal{E}$ is an Azumaya algebra over $\mathcal{A}$ of rank $r^{2}$ if there exists a faithfully flat $\mathcal{I}$-indexed $\mathcal{A}$-algebra $\mathcal{B}$ such that $\mathcal{E}$ splits over $\mathcal{B}$ with splitting module $M$ of rank $r$.

If we know that $\mathcal{E}$ is an Azumaya algebra over $\mathcal{A}$, then we can find a splitting module for $\mathcal{E}$ over $\mathcal{A}$ in certain case by the following proposition.

Proposition 2.9. Let $\mathcal{A}$ be a commutative $\mathcal{I}$-indexed $\mathcal{O}_{X}$-algebra and $\mathcal{E}$ an Azumaya algebra over $\mathcal{A}$ of rank $r^{2}$. If there exists a locally free $\mathcal{I}$-indexed $\mathcal{A}$ module $M$ of rank $r$ with a structure of $\mathcal{I}$-indexed left $\mathcal{E}$-module compatible with the given $\mathcal{I}$-indexed $\mathcal{A}$-module structure, then $\mathcal{E}$ splits over $\mathcal{A}$ with splitting module $M$.

Proof. See Corollary 2.5 of [11]. 


\section{The $p^{m+1}$-curvature map}

From this section, we are mainly concerned with log schemes. Our aim of this section is to construct the $p^{m+1}$-curvature map for a $\log$ smooth morphism $X \rightarrow S$ of fine $\log$ schemes defined over a field of positive characteristic, which generalizes the classical $p$-curvature map.

\section{1 - Logarithmic differential operators of higher level}

In this subsection, we briefly recall the log version of Berthelot's theory of differential operators of higher level which is studied by Montagnon. For more details, see [2] and [9].

Let us start with basics on log schemes [5]. A pre-log structure on a scheme $X$ is a pair $\left(\mathcal{M}_{X}, \alpha_{X}\right)$ where $\mathcal{M}_{X}$ is a sheaf of monoids on the étale site of $X$ and $\alpha_{X}$ is a homomorphism from $\mathcal{M}_{X}$ to the multiplicative monoid $\mathcal{O}_{X}$. A pre-log structure $\left(\mathcal{M}_{X}, \alpha_{X}\right)$ is a $\log$ structure if $\alpha_{X}$ induces an isomorphism from $\alpha_{X}^{-1}\left(\mathcal{O}_{X}^{*}\right)$ to $\mathcal{O}_{X}^{*}$. A log scheme is a pair of a scheme $X$ and a $\log$ structure $\left(\mathcal{M}_{X}, \alpha_{X}\right)$. We usually denote a $\log$ scheme by a single letter such as $X$ and the log structure of $X$ by $\mathcal{M}_{X}$. For a scheme $X$, the natural forgetful functor from the category of log structures on $X$ to that of pre-log structures on $X$ has a left adjoint functor (see (1.3) of [5]). We denote by $\left(\mathcal{M}_{X}^{a}, \alpha_{X}^{a}\right)$ the log structure defined by the image of a pre-log structure $\left(\mathcal{M}_{X}, \alpha_{X}\right)$ under this left adjoint functor. We call $\left(\mathcal{M}_{X}^{a}, \alpha^{a}\right)$ the log structure associated to $\left(\mathcal{M}_{X}, \alpha_{X}\right)$. A monoid $P$ is integral if the natural map from $P$ to the group $P^{g p}$ associated to $P$ is injective. It is fine if it is finitely generated and integral. A log scheme $X$ is fine if, étale locally on $X$, there exists a fine monoid $P$ with a morphism $P_{X} \rightarrow \mathcal{M}_{X}$ of monoids such that the log structure of $X$ is isomorphic to the log structure associated to a pre-log structure $P_{X} \rightarrow \mathcal{M}_{X} \rightarrow \mathcal{O}_{X}$. Here $P_{X}$ denotes the constant étale sheaf on $X$ defined by $P$. Fine log schemes form a category in an obvious way. This category has all finite projective limits (see (2.8) of [5]). A morphism of $\log$ schemes $f: X \rightarrow Y$ is strict if the natural morphism $f^{*} \mathcal{M}_{Y} \rightarrow \mathcal{M}_{X}$ induced by $f$ is an isomorphism, where $f^{*} \mathcal{M}_{Y}$ denotes the log structure on $X$ associated to a pre-log structure $f^{-1} \mathcal{M}_{Y} \rightarrow \mathcal{M}_{X} \rightarrow \mathcal{O}_{X}$. It is an exact closed immersion if it is strict and the underlying morphism of schemes is a closed immersion. One can define a log smooth (resp. log étale) morphism of fine log schemes in terms of local infinitesimal liftings in the category of fine log schemes (see Subsection 3 of [5]). Let $\left(X, \mathcal{M}_{X}\right) \rightarrow\left(S, \mathcal{M}_{S}\right)$ be a morphism of log schemes. Then we define the module of $\log$ differentials $\Omega_{\left(X, \mathcal{M}_{X}\right) /\left(S, \mathcal{M}_{S}\right)}^{1}$ by the quotient $\Omega_{X / S}^{1} \oplus\left(\mathcal{O}_{X} \otimes_{\mathrm{Z}} \mathcal{M}_{X}^{g p}\right) / N$. Here $\Omega_{X / S}^{1}$ is the module of differentials of the underlying morphism of schemes $X \rightarrow S$ and $N$ is the $\mathcal{O}_{X}$-submodule locally generated by the sections of the form $\left(d \alpha_{X}(a), 0\right)-\left(0, \alpha_{X}(a) \otimes a\right)$ with $a \in \mathcal{M}_{X}$ and $(0,1 \otimes a)$ with $a \in \operatorname{Im}\left(f^{*} \mathcal{M}_{S} \rightarrow \mathcal{M}_{X}\right)$. From now on, we simply denote the module of $\log$ differentials of $X \rightarrow S$ by $\Omega_{X / S}^{1}$. If $X \rightarrow S$ is a $\log$ smooth morphism of fine $\log$ schemes, then its module of log differentials $\Omega_{X / S}^{1}$ is a locally free $\mathcal{O}_{X}$-module of finite rank. 
From now on, all log schemes are assumed to be defined over $Z_{(p)}$. Let us recall the definition of the $m$-PD structure.

Definition 3.1. Let $X$ be a $\log$ scheme and $I$ a quasi-coherent ideal of $\mathcal{O}_{X}$. A divided power structure of level $m$ ( $m$-PD structure) on $I$ is a divided power ideal $(J, \gamma)$ of $\mathcal{O}_{X}$ such that

$$
I^{\left(p^{m}\right)}+p I \subset J \subset I
$$

and the divided power structure $\gamma$ on $J$ is compatible with the unique one on $p Z_{(p)}$. Here $I^{\left(p^{m}\right)}$ denotes the ideal of $\mathcal{O}_{X}$ generated by $p^{m}$-th powers of all sections of $I$. If $(J, \gamma)$ is an $m$-PD structure on $I$, we call $(I, J, \gamma)$ an $m$-PD ideal of $\mathcal{O}_{X}$ and call $(X, I, J, \gamma)$ an $m$-PD log scheme.

Let $(X, I, J, \gamma)$ be an $m$-PD log scheme. For each natural number $k$, we define the map $I \rightarrow \mathcal{O}_{X} ; f \mapsto f^{\{k\}(m)}$ by $f^{\{k\}(m)}:=f^{r} \gamma_{q}\left(f^{p^{m}}\right)$ where $k=p^{m} q+r$ and $0 \leq r<p^{m}$. These maps satisfy the following formulas (see p. 13 of [9]).

Proposition 3.2. Let $(X, I, J, \gamma)$ be an $m$-PD log scheme and $k, l$ be positive integers.

(1) For any $x \in I, x^{\{0\}(m)}=1, x^{\{1\}(m)}=x$, and $x^{\{k\}(m)} \in I$. Moreover if $k \geq p^{m}$, then $x^{\{k\}(m)} \in J$.

(2) For any $x \in I$ and $a \in \mathcal{O}_{X},(a x)^{\{k\}(m)}=a^{k} x^{\{k\}(m)}$.

(3) For any $x, y \in I,(x+y)^{\{k\}(m)}=\sum_{k^{\prime}+k^{\prime \prime}=k}\left\langle\begin{array}{c}k \\ k^{\prime}\end{array}\right\rangle x^{\left\{k^{\prime}\right\}(m)} y^{\left\{k^{\prime \prime}\right\}(m)}$.

(4) For any $x \in I, q_{k} ! x^{\{k\}(m)}=x^{k}$.

(5) For any $x \in I,\left(x^{\{k\}(m)}\right)^{\{l\}(m)}=\frac{q_{k l} !}{\left(q_{k} !\right)^{l} q_{l} !} x^{\{k l\}(m)}$.

In the following, we sometimes denote an element $f^{\{k\}(m)}$ simply by $f^{\{k\}}$, if there will be no confusions. For a while, we fix an $m$-PD fine log scheme $(S, \mathfrak{a}, \mathfrak{b}, \gamma)$ (i.e. $S$ is a fine $\log$ scheme) on which $p$ is locally nilpotent and a $\log$ smooth morphism $X \rightarrow S$ of fine $\log$ schemes. We assume that $\gamma$ extends to $X$ (for definition, see [2] Définition 1.3.2(1)). Note that $\gamma$ always extends to $X$ in the case $\mathfrak{b}=(p)$ (see [2] Définition 1.3.2(1)), which is the case of our interest.

To recall the sheaf of log differential operators of higher level, we need the log $m$-PD envelope. The construction of the $\log m$-PD envelope is the same as the classical case of level 0 , which we explain now: Let $i: X \hookrightarrow Y$ be an immersion of fine $\log$ schemes over $S$. Étale locally on $X$, we have a factorization $i=g \circ i^{\prime}$ with an exact closed immersion $i^{\prime}: X \hookrightarrow Z$ and a log étale morphism $g: Z \rightarrow Y$. Let $i^{\prime \prime}: X \hookrightarrow D$ be the usual $m$-PD envelope of $i^{\prime}$ (for definition, see [2]), and endow $D$ with the inverse image $\log$ structure of $Z$. Then, since $i^{\prime \prime}$ satisfies the obvious universal property, it descents to the exact closed immersion $X \hookrightarrow P_{X,(m)}(Y)$ with 
the $m$-PD structure globally on $X . P_{X,(m)}(Y)$ is called the log $m$-PD envelope of $i: X \hookrightarrow Y$.

Let us consider the diagonal immersion $X \rightarrow X \times_{S} X$. We simply denote its log $m$-PD envelope by $P_{X / S,(m)}$ and the defining ideal of $X \hookrightarrow P_{X / S,(m)}$ by $\bar{I}$. Then there exists the $m$-PD-adic filtration $\left\{\bar{I}^{\{n\}}\right\}_{n \in \mathbb{N}}$ associated to $\bar{I}$ (for definition, see [3] Définition A.3) which satisfies the following property.

If $x$ is a local section of $\bar{I}^{\{n\}}$, then $x^{\{k\}}$ is in $\bar{I}^{\{n k\}}$.

Let $\mathcal{P}_{X / S,(m)}$ denote the structure sheaf of $P_{X / S,(m)}$. For each natural number $n$, we denote by $\mathcal{P}_{X / S,(m)}^{n}$ the quotient sheaf $\mathcal{P}_{X / S,(m)} \bar{I}^{\{n+1\}}$ and by $P_{X / S,(m)}^{n}$ the closed subscheme of $P_{X / S,(m)}$ defined by $\bar{I}^{\{n+1\}}$. We have a sequence of surjective homomorphisms of sheaves

$$
\cdots \rightarrow \mathcal{P}_{X / S,(m)}^{n} \rightarrow \mathcal{P}_{X / S,(m)}^{n-1} \rightarrow \cdots \rightarrow \mathcal{P}_{X / S,(m)}^{1} \rightarrow \mathcal{P}_{X / S,(m)}^{0} .
$$

Let $p_{0}$ and $p_{1}$ (resp. $p_{0}^{n}$ and $p_{1}^{n}$ ) denote the first and second projection $P_{X / S,(m)} \rightarrow X$ (resp. $P_{X / S,(m)}^{n} \rightarrow X$ ) respectively.

Definition 3.3. Let $n, m$ be natural numbers. The sheaf of differential operators of level $m$ of order $\leq n$ is defined by

$$
\mathcal{D}_{X / S, n}^{(m)}:=\mathcal{H o m}_{\mathcal{O}_{X}}\left(p_{0 *}^{n} \mathcal{P}_{X / S,(m)}^{n}, \mathcal{O}_{X}\right) .
$$

The sheaf of differential operators of level $m$ is defined by

$$
\mathcal{D}_{X / S}^{(m)}:=\bigcup_{n \in \mathbb{N}} \mathcal{D}_{X / S, n}^{(m)} .
$$

REMARK 3.4. Since, for any $m^{\prime} \geq m$, an $m$-PD ideal can be considered as an $m^{\prime}$-PD ideal, $\left\{\mathcal{D}_{X / S}^{(m)}\right\}_{m \geq 0}$ naturally forms an inductive system.

$\mathcal{D}_{X / S}^{(m)}$ has the (non commutative) ring structure as follows. By using the universality of $m$-PD envelope, we obtain the canonical homomorphism of $\mathcal{O}_{X}$-algebras

$$
\delta_{m}^{n, n^{\prime}}: \mathcal{P}_{X / S,(m)}^{n+n^{\prime}} \rightarrow \mathcal{P}_{X / S,(m)}^{n} \otimes_{\mathcal{O}_{X}} \mathcal{P}_{X / S,(m)}^{n^{\prime}}
$$

for each natural number $n, n^{\prime}$, which is induced by the projection $X \times_{S} X \times_{S} X \rightarrow$ $X \times_{S} X$ to the first and the third factors (for precise definition of $\delta_{m}^{n, n^{\prime}}$, see Subsection 2.3.2 of [9]). For each $\Phi \in \mathcal{D}_{X / S, n}^{(m)}$ and $\Psi \in \mathcal{D}_{X / S, n^{\prime}}^{(m)}$, we define the product $\Phi \cdot \Psi \in \mathcal{D}_{X / S, n+n^{\prime}}^{(m)}$ by

$$
\mathcal{P}_{X / S,(m)}^{n+n^{\prime}} \stackrel{\delta_{m}^{n, n^{\prime}}}{\longrightarrow} \mathcal{P}_{X / S,(m)}^{n} \otimes_{\mathcal{O}_{X}} \mathcal{P}_{X / S,(m)}^{n^{\prime}} \stackrel{\operatorname{id} \otimes \Psi}{\longrightarrow} \mathcal{P}_{X / S,(m)}^{n} \stackrel{\Phi}{\longrightarrow} \mathcal{O}_{X}
$$

This is well-defined and $\mathcal{D}_{X / S}^{(m)}$ forms a sheaf of non commutative $\mathcal{O}_{X}$-algebras on $X$. 
REMARK 3.5. Let $\mathcal{E}$ be an $\mathcal{O}_{X}$-module. Then a $\log m$-PD stratification on $\mathcal{E}$ is a family of $\mathcal{P}_{X / S,(m)}^{n}$-linear isomorphisms $\varepsilon_{n}: p_{0}^{n *} \mathcal{E} \cong p_{1}^{n *} \mathcal{E}$ satisfying the usual cocycle conditions. As is the same with the classical case, giving a $\mathcal{D}_{X / S}^{(m)}$-action on $\mathcal{E}$ extending its $\mathcal{O}_{X}$-module structure is equivalent to giving a $\log m$-PD stratification on $\mathcal{E}$.

Finally we recall the local description of $\mathcal{D}_{X / S}^{(m)}$. Let $j$ denote the log $m$-PD envelope $X \hookrightarrow P_{X / S,(m)}$ of the diagonal $X \rightarrow X \times_{S} X$. We have an exact sequence

$$
0 \rightarrow j^{-1}(1+\bar{I}) \stackrel{\lambda}{\longrightarrow} j^{-1} \mathcal{M}_{P_{X / S,(m)}} \stackrel{j^{*}}{\longrightarrow} \mathcal{M}_{X} \rightarrow 0,
$$

where $\lambda$ is the restriction of the log structure $j^{-1}\left(\alpha_{P_{X / S,(m)}}^{-1}\right): j^{-1}\left(\mathcal{P}_{X / S,(m)}^{*}\right) \rightarrow$ $j^{-1}\left(\mathcal{M}_{P_{X / S,(m)}}^{*}\right)$. For any section $a \in \mathcal{M}_{X}, p_{0}^{*}(a)$ and $p_{1}^{*}(a)$ have the same image in $\mathcal{M}_{X}$. Thus, from the exact sequence (2), there exists a unique section $\mu_{(m)}(a) \in j^{-1}(1+\bar{I})$ such that $p_{1}^{*}(a)=p_{0}^{*}(a) \cdot \lambda\left(\mu_{(m)}(a)\right)$. Log smoothness of $X \rightarrow S$ implies that, étale locally on $X$, there is a logarithmic system of coordinates $m_{1}, \ldots, m_{r} \in \mathcal{M}_{X}^{g p}$, that is, a system of sections such that the set $\left\{d \log m_{1}, \ldots, d \log m_{r}\right\}$ forms a basis of the $\log$ differential module $\Omega_{X / S}^{1}$ of $X$ over $S$. We define $\eta_{i}^{(m)}:=\mu_{(m)}\left(m_{i}\right)-1$ and $\underline{\eta}^{\{\underline{k}\}(m)}:=\prod_{i:=1}^{r} \eta_{i}^{\left\{k_{i}\right\}(m)}$ for each multi-index $\underline{k} \in \mathbb{N}^{r}$.

Proposition-Definition 3.6. We regard $\mathcal{P}_{X / S,(m)}^{n}$ as an $\mathcal{O}_{X}$-module via $p_{0 *}^{n}$. Then the set $\left\{\underline{\eta}\{\underline{\underline{k}\}(m)}|| \underline{k} \mid \leq n\}\right.$ forms a local basis of $\mathcal{P}_{X / S,(m)}^{n}$ over $\mathcal{O}_{X}$. We denote the dual basis of $\left\{\underline{\eta}^{\{\underline{k}\}(m)}|| \underline{k} \mid \leq n\right\}$ by $\left\{\underline{\partial}_{\langle\underline{k}\rangle(m)}|| \underline{k} \mid \leq n\right\}$. We also denote $\underline{\eta}^{\{\underline{k}\}(m)}$ (resp. $\left.\underline{\partial}_{\langle\underline{k}\rangle(m)}\right)$ by $\underline{\eta}^{\{\underline{k}\}}\left(\right.$ resp. $\left.\underline{\partial}_{\langle\underline{k}\rangle}\right)$ simply, if there will be no confusions.

Proof. See Proposition 2.2.1 of [9].

Proposition 3.7. Let $X \rightarrow S$ be a log smooth morphism of fine log schemes. Assume that we are given a logarithmic system of coordinates $m_{1}, \ldots, m_{r} \in \mathcal{M}_{X}^{g p}$.

(1) $\mathcal{D}_{X / S}^{(m)}$ is locally generated by $\left\{\underline{\partial}_{\left\langle\underline{\varepsilon}_{i}\right\rangle}, \underline{\partial}_{\left\langle\underline{p}_{i}\right\rangle}, \ldots, \underline{\partial}_{\left\langle p^{m} \underline{\varepsilon}_{i}\right\rangle} \mid 1 \leq i \leq r\right\}$ as an $\mathcal{O}_{X^{-}}$ algebra.

(2) We have

$$
\left.\underline{\partial}_{\left\langle\underline{k}^{\prime}\right\rangle} \cdot \underline{\partial}_{\left\langle\underline{k}^{\prime \prime}\right\rangle}=\sum_{\underline{k}=\sup \left\{\underline{k}^{\prime}, \underline{k}^{\prime \prime}\right\}}^{\underline{k}^{\prime}+\underline{k}^{\prime \prime}} \frac{\underline{k} !}{\left(\underline{k}^{\prime}+\underline{k}^{\prime \prime}-\underline{k}\right) !\left(\underline{k}-\underline{k^{\prime}}\right) !\left(\underline{k}-\underline{k}^{\prime \prime}\right) !} \frac{\underline{q}_{k^{\prime}} ! \underline{q}_{\underline{k}}^{\prime \prime} !}{\underline{q}_{\underline{k}} !} \underline{\partial}_{\langle\underline{k}}\right\rangle .
$$

In particular, $\underline{\partial}_{\langle\underline{k}\rangle} \cdot \underline{\partial}_{\left\langle\underline{k}^{\prime}\right\rangle}=\underline{\partial}_{\left\langle\underline{k}^{\prime}\right\rangle} \cdot \underline{\partial}_{\langle\underline{k}\rangle}$ holds.

(3) For any $x \in \mathcal{O}_{X}$, we have

$$
\underline{\partial}_{\langle\underline{k}\rangle} \cdot x=\sum_{\underline{i} \leq \underline{k}}\left\{\frac{k}{\underline{i}}\right\} \underline{\partial}_{\langle\underline{k}-\underline{i}\rangle}(x) \underline{\partial}_{\langle\underline{i}\rangle} \quad \text { in } \mathcal{D}_{X / S}^{(m)} .
$$

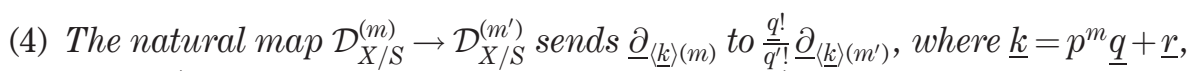
$\underline{k^{\prime}}=p^{m^{\prime}} \underline{q}^{\prime}+\underline{r^{\prime}}$ with $0 \leq \underline{r}<p^{m}$ and $0 \leq \underline{r^{\prime}}<p^{\underline{m}^{i}}$. 
Proof. (1) See Proposition 2.3.1 of [9]. (2) See Lemme 2.3.4 of [9]. (3) See (2.5) of [9]. (4) See (2.6) of [9].

We prove the following lemma needed later.

LEMMA 3.8. Let $X \rightarrow S$ be a log smooth morphism of fine log schemes defined over Z/pZ. For any $\underline{k} \in \mathbb{N}^{r}, l \in \mathbb{N}$ and $1 \leq i \leq r$, we have

$$
\underline{\partial}_{\left\langle p^{m+1} \underline{k}\right\rangle} \cdot \underline{\partial}_{\left\langle\underline{\varepsilon}_{i}\right\rangle}=\underline{\partial}_{\left\langle p^{m+1} \underline{k}+l_{i}\right\rangle} \cdot
$$

Proof. We may assume $1 \leq l \leq p^{m+1}$. When $k_{i}=0$, the assertion follows easily from Proposition 3.7 (2). Thus we may also assume $k_{i} \geq 1$. By Proposition 3.7 (2), we have

$$
\text { (⿻) } \underline{\partial}_{\left\langle p^{m+1} \underline{k}\right\rangle} \cdot \underline{\partial}_{\left\langle\underline{l}_{i}\right\rangle}=\sum_{s=0}^{l} \frac{\left(p^{m+1} k_{i}+s\right) !}{(l-s) ! s !\left(p^{m+1} k_{i}-l+s\right) !} \frac{\left(p k_{i}\right) ! q_{l} !}{\left(p k_{i}+q_{s}\right) !} \underline{\partial}_{\left\langle p^{m+1} \underline{k}+s \underline{\varepsilon}_{i}\right\rangle} .
$$

We put

$$
A:=\frac{\left(p^{m+1} k_{i}+s\right) !}{(l-s) ! s !\left(p^{m+1} k_{i}-l+s\right) !} \in Z \text { and } B:=\frac{\left(p k_{i}\right) ! q_{l} !}{\left(p k_{i}+q_{s}\right) !} \in \mathbb{Q} \text {. }
$$

First, we consider the case $s=l$. Then, we have

$$
\begin{aligned}
A \cdot B & =\frac{\left(p^{m+1} k_{i}+l\right) !}{l !\left(p^{m+1} k_{i}\right) !} \frac{\left(p k_{i}\right) ! q_{l} !}{\left(p k_{i}+q_{l}\right) !} \\
& =\prod_{j=1}^{l}\left(1+\frac{p^{m+1} k_{i}}{j}\right) \cdot \prod_{j=1}^{q_{l}}\left(1+\frac{p k_{i}}{j}\right)^{-1}
\end{aligned}
$$

Since $1+\frac{p^{m+1} k_{i}}{j} \in 1+p Z_{(p)}$ if $1 \leq j \leq p^{m+1}-1,1+\frac{p^{m+1} k_{i}}{p^{m+1}}=1+k_{i} \quad$ if $j=p^{m+1}$, $\left(1+\frac{p k_{i}}{j}\right)^{-1} \in 1+p Z_{(p)}$ if $1 \leq j \leq p-1$ and $\left(1+\frac{p k_{i}}{p}\right)^{-1}=\left(1+k_{i}\right)^{-1}$ if $j=p$, we have $A \cdot B \in 1+p Z_{(p)}$ and thus $A \cdot B \equiv 1 \bmod p$. Next, we consider the case $0 \leq s \leq l-1, l=p^{m+1}$. Then, we have

$$
B=\frac{\left(p k_{i}\right) ! q_{l} !}{\left(p k_{i}+q_{s}\right) !}=\prod_{j=1}^{q_{s}} \frac{1}{\left(p k_{i}+j\right)} \cdot p ! \in p Z_{(p)} .
$$

Hence $A \cdot B \in p Z_{(p)}$. We thus have $A \cdot B \equiv 0 \bmod p$. Finally, we consider the case $0 \leq s \leq l-1,0 \leq l \leq p^{m+1}-1$. Then, we have

$$
B=\prod_{j=1}^{q_{s}} \frac{1}{\left(p k_{i}+j\right)} \cdot q_{l} ! \in Z_{(p)}
$$

Let $v: \mathbb{Q}^{*} \rightarrow Z$ denote the normalized $p$-adic valuation. For any $n \in \mathbb{N}$, it is 
known that $(p-1) v(n !)=n-\sigma(n)$, where $\sigma(n):=\sum_{j} a_{j}$ if $n=\sum_{j} a_{j} p^{j}$. Thus, we have

$$
\begin{aligned}
(p-1) v(A) & =\sigma(l-s)+\sigma(s)+\sigma\left(p^{m+1} k_{i}-l+s\right)-\sigma\left(p^{m+1} k_{i}+s\right) \\
& =\sigma(l-s)+\sigma\left(p^{m+1} k_{i}-l+s\right)-\sigma\left(p^{m+1} k_{i}\right) \\
& >0 .
\end{aligned}
$$

Hence $A \cdot B \in p Z_{(p)}$. We thus have $A \cdot B \equiv 0 \bmod p$. The assertion follows from these calculations and (⿳). $)$.

\section{2 - The $p^{m+1}$-curvature map}

Throughout this subsection, all the log schemes are assumed to be defined over $Z / p Z$. Let us introduce some notations. For a log scheme $X, F_{X}$ denotes the $(m+1)$-st iterate of its absolute Frobenius. For a morphism $X \rightarrow S$ of fine log schemes, we consider the following commutative diagram:

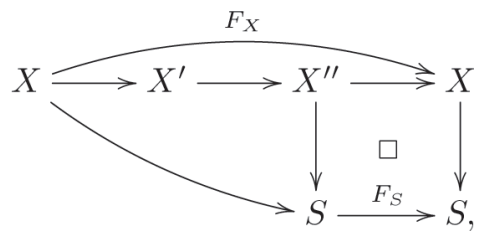

where $X^{\prime \prime}$ is the fiber product in the category of fine log schemes, and the morphism $X \rightarrow X^{\prime}$ (denoted by $F_{X / S}$ and called the $((m+1)$-st) relative Frobenius morphism) is uniquely determined by the requirement that the morphism $F_{X / S}$ is purely inseparable and $X^{\prime} \rightarrow X^{\prime \prime}$ is log étale (see Proposition 4.10 of [5]). We denote the composition $X^{\prime} \rightarrow X^{\prime \prime} \rightarrow X$ by $\pi_{X / S}$ or simply by $\pi$. We also denote $X^{\prime}$ by $X^{(m+1)}$, if there is a risk of confusion.

First we prove the log level $m$ version of Mochizuki's theorem which is used to construct the $p^{m+1}$-curvature map (see also Proposition 3.2 of [4] and Proposition A.7 of [11]).

THEOREM 3.9. Let $X \rightarrow S$ be a log smooth morphism of fine log schemes. Let $P_{X / S,(m)}$ (resp. $\left.Y\right)$ be the log m-PD envelope (resp. the log formal neighborhood) of the diagonal immersion $X \rightarrow X \times_{S} X$ and $\bar{I}$ (resp. I) its defining ideal. Let $\mathcal{P}_{X / S,(m)}$ denote the structure sheaf of $P_{X / S,(m)}$. Then there is an isomorphism of $\mathcal{O}_{X}$-modules

$$
\alpha: F_{X / S}^{*} \Omega_{X^{\prime} / S}^{1} \rightarrow \bar{I} /\left(\bar{I}^{\left\{p^{m+1}+1\right\}}+I \mathcal{P}_{X / S,(m)}\right)
$$

such that, for any $\xi \in I$ with image $\omega \in I / I^{2} \cong \Omega_{X / S}^{1}$,

$$
\alpha\left(1 \otimes \pi^{*} \omega\right)=\xi^{\left\{p^{m+1}\right\}} .
$$


Proof. First we show that the map $\alpha^{\prime}: I \rightarrow \bar{I} / I \mathcal{P}_{X / S,(m)}$ defined by

$$
\begin{array}{ccc}
I & \longrightarrow & \bar{I} / I \mathcal{P}_{X / S,(m)} \\
\xi & \longmapsto & \xi^{\left\{p^{m+1}\right\}}
\end{array}
$$

is $F_{X}^{*}$-linear and zero on $I^{2}$. If $\xi$ and $\tau$ are local sections of $I$, by Proposition 3.2 (3) we have

$$
(\xi+\tau)^{\left\{p^{m+1}\right\}}=\xi^{\left\{p^{m+1}\right\}}+\tau^{\left\{p^{m+1}\right\}}+\sum_{\substack{i+j=p^{m+1} \\
i, j>0}}\left\langle\begin{array}{c}
p^{m+1} \\
i
\end{array}\right\rangle \xi^{\{i\}} \tau^{\{j\}} .
$$

Since $0<i, j<p^{m+1}$, we have $q_{i}, q_{j}<p$, where $q_{i}, q_{j}$ are as in Subsection 1.3. Therefore $q_{i}$ ! and $q_{j}$ ! are invertible. From Proposition 3.2 (4) we have $\xi^{\{i\}} \tau^{\{j\}}=\left(q_{i} ! q_{j} !\right)^{-1} \xi^{i} \tau^{j} \in I \mathcal{P}_{X / S,(m)}$. It follows that the last term in the sum is in $I \mathcal{P}_{X / S,(m)}$ and we see the additivity of $\alpha^{\prime}$. Similarly, the fact that $\alpha^{\prime}$ is $F_{X}^{*}$-linear and zero on $I^{2}$ follows from Proposition 3.2 (2), (5). We thus obtain the $\mathcal{O}_{X}$-linear map

$$
\alpha: F_{X / S}^{*} \Omega_{X^{\prime} / S}^{1} \cong F_{X}^{*} \Omega_{X / S}^{1} \rightarrow \bar{I} /\left(\bar{I}^{\left\{p^{m+1}+1\right\}}+I \mathcal{P}_{X / S,(m)}\right)
$$

which satisfies (3). Let us show that $\alpha$ is an isomorphism. Since the assertion is étale local on $X$, we may assume that we have a logarithmic system of coordinates $m_{1}, \ldots, m_{r} \in \mathcal{M}_{X}^{g p}$. Then the left hand side is isomorphic to $\bigoplus_{i=1}^{r} \mathcal{O}_{X}\left(1 \otimes \pi^{*} d \log m_{i}\right)$. On the other hand, by Proposition-Definition 3.6, $\bar{I} / \bar{I}^{\left\{p^{m+1}+1\right\}}$ is freely generated by $\left\{\underline{\eta}^{\{i\}}|1 \leq| \underline{i} \mid \leq p^{m+1}\right\}$ as an $\mathcal{O}_{X}$-module and the image of $I \mathcal{P}_{X / S,(m)}$ under the map $I \mathcal{P}_{X / S,(m)} \rightarrow \bar{I} / \bar{I}^{\left\{p^{m+1}+1\right\}}$ is generated by $\left\{\eta_{j} \underline{\eta}^{\{\underline{i}\}}|0 \leq| \underline{i} \mid \leq p^{m+1}-1,1 \leq j \leq r\right\}$ as an $\mathcal{O}_{X}$-module. Actually $\bar{I} /\left(\bar{I}^{\left\{p^{m+1}+1\right\}}+I \mathcal{P}_{X / S,(m)}\right)$ is freely generated by $\left\{\eta_{i}^{\left\{p^{m+1}\right\}} \mid 1 \leq i \leq r\right\}$ as an $\mathcal{O}_{X}$-module. So the right hand side is isomorphic to $\bigoplus_{i=1}^{r} \mathcal{O}_{X} \eta_{i}^{\left\{p^{m+1}\right\}}$ and, by construction, $\alpha$ sends $1 \otimes \pi^{*} d \log m_{i}$ to $\eta_{i}^{\left\{p^{m+1}\right\}}$. This completes the proof.

Let $a$ denote the map defined by the composite

$$
\mathcal{P}_{X / S,(m)}^{n} \rightarrow \mathcal{O}_{X} \rightarrow \mathcal{P}_{X / S,(m)}^{n},
$$

where the first map is the natural projection and the second one is the structural morphism $p_{0}^{n *}$. Now, we are ready to define the $p^{m+1}$-curvature map.

DeFinition 3.10. Let $X \rightarrow S$ be a $\log$ smooth morphism of fine $\log$ schemes. Let $\mathcal{T}_{X^{\prime} / S}:=\mathcal{H o m}_{\mathcal{O}_{X^{\prime}}}\left(\Omega_{X^{\prime} / S}^{1}, \mathcal{O}_{X^{\prime}}\right)$ denote the log tangent bundle on $X^{\prime}$. We define the $\operatorname{map} \beta: \mathcal{T}_{X^{\prime} / S} \rightarrow F_{X / S *} \mathcal{D}_{X / S}^{(m)}$ by sending $D \in \mathcal{T}_{X^{\prime} S}$ to the composition of maps

$$
\mathcal{P}_{X / S,(m)}^{p^{m+1}} \stackrel{y \mapsto y-a(y)}{\longrightarrow} \bar{I} / \bar{I}^{\left\{p^{m+1}\right\}} \rightarrow \bar{I} /\left(\bar{I}^{\left\{p^{m+1}+1\right\}}+I \mathcal{P}_{X, m}\right) \stackrel{\cong}{\cong} F_{X / S}^{*} \Omega_{X^{\prime} / S}^{1} \stackrel{F_{X / S}^{*} D}{\longrightarrow} \mathcal{O}_{X},
$$

where the second map is the natural projection and the third one is the isomorphism in Theorem 3.9. We call it the $p^{m+1}$-curvature map.

The local description of the $p^{m+1}$-curvature map is the following. 
Proposition 3.11. Let $X \rightarrow S$ be a log smooth morphism of fine log schemes. Assume that we are given a logarithmic system of coordinates $m_{1}, \ldots, m_{r} \in \mathcal{M}_{X}^{g p}$. Let $\left\{\xi_{i}^{\prime} \mid 1 \leq i \leq r\right\}$ denote the dual basis of $\left\{\pi^{*} d \log m_{i} \mid 1 \leq i \leq r\right\}$. Then $\beta$ sends $\xi_{i}^{\prime}$ to $\underline{\partial}_{\left\langle p^{m+1} \underline{\varepsilon}_{i}\right\rangle}$.

Proof. We calculate that $\beta\left(\xi_{i}^{\prime}\right)$ sends $\underline{\eta}^{\{\underline{k}\}}$ to 1 if $\underline{k}=p^{m+1} \underline{\varepsilon}_{i}$ and 0 otherwise by construction of $\beta$, thereby completing the proof.

REMARK 3.12. When $m$ is equal to 0 , our $p^{m+1}$-curvature map is the usual $p$ curvature map ([10] Proposition 1.7). If the $\log$ structure of $X$ is trivial, then our $p^{m+1}$-curvature map coincides with the $p^{m}$-curvature map studied in [4] section 3.

\section{Azumaya algebra property}

The goal of this section is the Azumaya algebra property of the indexed version of the sheaf of log differential operators $\tilde{\mathcal{D}}_{X / S}^{(m)}=\mathcal{A}_{X}^{g p} \otimes_{\mathcal{O}_{X}} \mathcal{D}_{X / S}^{(m)}$ defined by Montagnon [9]. We also study the Azumaya nature of $\tilde{\mathcal{D}}_{X^{(m)} / S}^{(0)}=\mathcal{B}_{X / S}^{(m)} \otimes_{\mathcal{O}_{X^{(m)}}} \mathcal{D}_{X^{(m)} / S}^{(0)}$, which is also introduced by Montagnon. At first we give a review of the canonical indexed algebra $\mathcal{A}_{X}^{g p}$ associated to the $\log$ structure of $X$ introduced by Lorenzon [5] and Montagnon's $\tilde{\mathcal{D}}_{X / S}^{(m)}$ and $\tilde{\mathcal{D}}_{X^{(m) / S}}^{(0)}$.

\section{1 - Indexed algebra associated to a log structure}

\subsection{1 - The $\mathcal{I}_{X}^{g p}$-indexed algebra $\tilde{\mathcal{D}}_{X / S}^{(m)}$}

First we recall the definition of $\mathcal{A}_{X}^{g p}$ (I, 3.1 of [5]). Let $X$ be a fine log scheme. We consider the extension of sheaves of abelian groups

$$
0 \longrightarrow \mathcal{O}_{X}^{*} \longrightarrow \mathcal{M}_{X}^{g p} \stackrel{\delta}{\longrightarrow} \mathcal{I}_{X}^{g p} \longrightarrow 0 .
$$

Here $\mathcal{I}_{X}^{g p}$ is the quotient sheaf $\mathcal{M}_{X}^{g p} / \mathcal{O}_{X}^{*}$. We define $\mathcal{A}_{X}^{g p}$ as the contracted product $\mathcal{M}_{X}^{g p} \wedge_{\mathcal{O}_{X}^{*}} \mathcal{O}_{X}$ which is the quotient of $\mathcal{M}_{X}^{g p} \times \mathcal{O}_{X}$ by the equivalence relation $(a x, y) \sim(x, a y)$ where $a, x, y$ are local sections of $\mathcal{O}_{X}^{*}, \mathcal{M}_{X}^{g p}$ and $\mathcal{O}_{X}$ respectively. The projection $\mathcal{M}_{X}^{g p} \times \mathcal{O}_{X} \rightarrow \mathcal{M}_{X}^{g p} \rightarrow \mathcal{I}_{X}^{g p}$ induces a map $\mathcal{A}_{X}^{g p} \rightarrow \mathcal{I}_{X}^{g p}$ which makes $\mathcal{A}_{X}^{g p}$ an $\mathcal{I}_{X}^{g p}$-indexed $\mathcal{O}_{X}$-module. For a local section $i$ of $\mathcal{I}_{X}^{g p}$, the fiber $\mathcal{M}_{X, i}^{g p}$ of $\mathcal{M}_{X}^{g p} \rightarrow \mathcal{I}_{X}^{g p}$ at $i$ is an $\mathcal{O}_{X}^{*}$-torsor. This implies that the fiber $\mathcal{A}_{X, i}^{g p}$ of $\mathcal{A}_{X}^{g p} \rightarrow \mathcal{I}_{X}^{g p}$ at $i$ is an invertible $\mathcal{O}_{X^{-}}$ module. $\mathcal{A}_{X}^{g p}$ has a multiplication map induced by the addition map $\mathcal{M}_{X}^{g p} \times \mathcal{M}_{X}^{g p} \rightarrow$ $\mathcal{M}_{X}^{g p}$ over $\mathcal{I}_{X}^{g p} \times \mathcal{I}_{X}^{g p} \rightarrow \mathcal{I}_{X}^{g p}$. Hence $\mathcal{A}_{X}^{g p}$ forms an $\mathcal{I}_{X}^{g p}$-indexed $\mathcal{O}_{X}$-algebra, called the $\mathcal{I}_{X}^{g p}$-indexed algebra associated to the log structure.

Next let us recall the definition of the section $e_{s}$ of $\mathcal{A}_{X}^{g p}$ associated to a section $s$ of $\mathcal{M}_{X}^{g p}$. For each étale open $U$ of $X$ and a section $s \in \mathcal{M}_{X}^{g p}(U), s$ trivializes the $\mathcal{O}_{X^{-}}^{*}$ 
torsor $\mathcal{M}_{X, \delta(s)}^{g p}$. Thus it gives a basis $e_{s}:=\overline{(s, 1)}$ of the invertible $\mathcal{O}_{U^{-m o d u l e}} \mathcal{A}_{X, \delta(s)}^{g p}$. Then

$$
e_{0}=1, e_{s} e_{t}=e_{s+t}, a e_{s}=e_{a s}
$$

for $s, t \in \mathcal{M}_{X}^{g p}(U)$ and $a \in \mathcal{O}_{X}^{*}(U)$.

The construction of $\mathcal{A}_{X}^{g p}$ is functorial in the following sense. For a morphism $f: X \rightarrow Y$ of fine log schemes, we have a commutative diagram

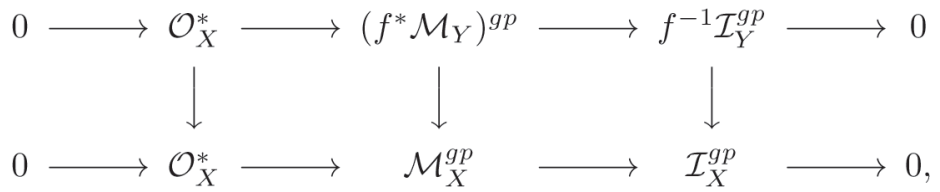

where vertical arrows are isomorphisms if $f$ is strict. This induces a commutative diagram

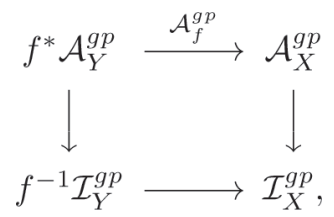

where horizontal arrows are isomorphisms if $f$ is strict. So we get the following proposition.

Proposition 4.1. If $f: X \rightarrow Y$ is a strict morphism of fine log schemes, then $\mathcal{A}_{f}^{g p}: f^{*} \mathcal{A}_{Y}^{g p} \rightarrow \mathcal{A}_{X}^{g p}$ is an isomorphism of $\mathcal{I}_{X}^{g p}$-indexed algebras.

Next we recall the definition of $\mathcal{I}_{X}^{g p}$-indexed left $\mathcal{D}_{X / S}^{(m)}$-module structure on $\mathcal{A}_{X}^{g p}$ (Subsection 4.1.1 of [9]). Let $*$ be the trivial sheaf of abelian groups on $X$. We naturally regard $\mathcal{D}_{X / S}^{(m)}$ as a $*$-indexed $\mathcal{O}_{X}$-algebra and $\mathcal{I}_{X}^{g p}$ as a sheaf of $*$-sets. Since the natural projections $p_{i}^{n}: P_{X,(m)}^{n} \rightarrow X$ with $i=0,1$ are strict, we have the isomorphism of $\mathcal{I}_{X}^{g p}$-indexed $\mathcal{O}_{X}$-algebras

$$
\varepsilon_{n}: p_{1}^{n *} \mathcal{A}_{X}^{g p} \cong \mathcal{A}_{P_{X / S,(m)}^{n}} \cong p_{0}^{n *} \mathcal{A}_{X}^{g p}
$$

These isomorphisms are compatible with respect to $n$ by construction and satisfy the cocycle condition. Hence we can define an $\mathcal{I}_{X}^{g p}$-indexed left $\mathcal{D}_{X / S}^{(m)}$-module structure on $\mathcal{A}_{X}^{g p}$ by

$$
\begin{aligned}
& \mathcal{D}_{X / S}^{(m)} \times \mathcal{A}_{X}^{g p} \hookrightarrow \mathcal{D}_{X / S}^{(m)} \times p_{1}^{*} \mathcal{A}_{X}^{g p} \stackrel{\mathrm{id} \times \varepsilon}{\longrightarrow} \mathcal{D}_{X / S}^{(m)} \times p_{0}^{*} \mathcal{A}_{X}^{g p} \\
& \hookrightarrow \mathcal{H o m}_{\mathcal{O}_{X}}\left(\mathcal{P}_{X / S,(m)}, \mathcal{O}_{X}\right) \times \mathcal{A}_{X}^{g p} \otimes_{\mathcal{O}_{X}} \mathcal{P}_{X / S,(m)} \rightarrow \mathcal{A}_{X}^{g p} .
\end{aligned}
$$

By calculation with the section $e_{s}\left(s \in \mathcal{I}_{X}^{g p}\right)$, one can see that the action of $\mathcal{D}_{X / S}^{(m)}$ on $\mathcal{A}_{X}^{g p}$ satisfies the Leibniz formula (for more details, see Subsection 4.1 of [9]). 
Therefore we have the following nontrivial ring structure on $\tilde{\mathcal{D}}_{X / S}^{(m)}$ which is a central object in this article.

Proposition-Definition 4.2. Let $X \rightarrow S$ be a log smooth morphism of fine log schemes. Let $\tilde{\mathcal{D}}_{X / S}^{(m)}$ denote the $\mathcal{I}_{X}^{g p}$-indexed $\mathcal{O}_{X}$-module $\mathcal{A}_{X}^{g p} \otimes_{\mathcal{O}_{X}} \mathcal{D}_{X / S}^{(m)}$. Then there exists a unique $\mathcal{I}_{X}^{g p}$-indexed $\mathcal{O}_{X}$-algebra structure on $\tilde{\mathcal{D}}_{X / S}^{(m)}$ such that the maps $\mathcal{A}_{X}^{g p} \rightarrow \tilde{\mathcal{D}}_{X / S}^{(m)} ; a \mapsto a \otimes 1$ and $\mathcal{D}_{X / S}^{(m)} \rightarrow \tilde{\mathcal{D}}_{X / S}^{(m)} ; P \mapsto 1 \otimes P$ are homomorphisms and that for any $a \in \mathcal{A}_{X}^{g p}, P \in \mathcal{D}_{X / S}^{(m)}$ and $k \in \mathbb{N}^{r}$, we have the relations $a \otimes P=$ $(a \otimes 1)(1 \otimes P)$ and

$$
\left(1 \otimes \underline{\partial}_{\langle\underline{k}\rangle}\right)(a \otimes 1)=\sum_{\underline{i} \leq \underline{k}}\left\{\begin{array}{l}
\underline{k} \\
\underline{i}
\end{array}\right\}\left(\underline{\partial}_{\langle\underline{k}-\underline{i}\rangle} \cdot a\right) \otimes \underline{\partial}_{\langle\underline{i}\rangle} .
$$

Here $\left\{\underline{\partial}_{\langle\underline{k}\rangle}\right\}$ is as in Proposition-Definition 3.6.

Proof. See Subsection 4.1 of [9].

REMark 4.3. In [9], Montagnon defines $\tilde{\mathcal{D}}_{X / S}^{(m)}$ by $\mathcal{A}_{X}^{g p} \otimes_{\mathcal{O}_{X, \mathcal{I}}} \mathcal{D}_{X / S, \mathcal{I}}^{(m)}$, where $\mathcal{O}_{X, \mathcal{I}}$ $\left(\right.$ resp. $\left.\mathcal{D}_{X / S, \mathcal{I}}^{(m)}\right)$ denotes an $\mathcal{I}$-indexed $\mathcal{O}_{X}$-module $\mathcal{O}_{X} \times \mathcal{I}\left(\right.$ resp. $\left.\mathcal{D}_{X / S}^{(m)} \times \mathcal{I}\right)$ with the second projection. So, in Montagnon's definition of $\tilde{\mathcal{D}}_{X / S}^{(m)}$, if we take a section $a \otimes P \in \tilde{\mathcal{D}}_{X / S}^{(m)}$, then $P$ may have a nontrivial index. It seems to us that $P$ must have the trivial index. For example, equation (4) in the following Proposition 4.4 should actually be considered as an equation in $\tilde{\mathcal{D}}_{X / S}^{(m)}$. If $\underline{\partial}_{\langle\underline{k}\rangle}$ has a nontrivial index, then (4) does not make sense because both sides of the equation must have the same index. If we define $\tilde{\mathcal{D}}_{X / S}^{(m)}:=\mathcal{A}_{X}^{g p} \otimes_{\mathcal{O}_{X}} \mathcal{D}_{X / S}^{(m)}$ by regarding $\mathcal{D}_{X / S}^{(m)}$ as a $*$-indexed $\mathcal{O}_{X}$-algebra in a natural way, then equation (4) makes sense (both sides have the same index $\prod_{i} m_{i}^{j_{i}}$ ). Therefore, it seems to us that the natural definition of $\tilde{\mathcal{D}}_{X / S}^{(m)}$ is $\mathcal{A}_{X}^{g p} \otimes_{\mathcal{O}_{X}} \mathcal{D}_{X / S}^{(m)}$. However, this is not serious. Actually, if we replace in [9] $\mathcal{A}_{X}^{g p} \otimes_{\mathcal{O}_{X, \mathcal{I}}} \mathcal{D}_{X / S, \mathcal{I}}^{(m)}$ by $\mathcal{A}_{X}^{g p} \otimes_{\mathcal{O}_{X}} \mathcal{D}_{X / S}^{(m)}$ (and also modify the definition of $\mathcal{B}_{X / S}^{(m)}$ and $\mathbb{F}(\mathcal{E})$, see PropositionDefinition 4.7 and Subsection 6.1 respectively), then all equations in [9] make sense and all the proofs are correct without any essential changes.

Finally we recall the following formula needed later. Let $m_{1}, \ldots, m_{r} \in \mathcal{M}_{X}^{g p}$ be a logarithmic system of coordinates. For $i \in\{1,2, \ldots, r\}$ and a multi-index $\underset{j}{j}$, we put $\theta_{i}:=e_{m_{i}}$ and $\underline{\theta}^{j}:=\prod_{i} \theta_{i}^{j_{i}}$.

Proposition 4.4. For $k \in \mathbb{N}^{r}$,

$$
\underline{\partial}_{\langle\underline{k}\rangle} \cdot \underline{\theta}^{j}=\underline{q}_{\underline{k}} !\left(\frac{j}{\underline{k}}\right) \underline{\theta}^{\underline{j}} .
$$

Proof. See Lemme 4.2.3 of [9]. 


\subsection{2 - The $\mathcal{I}_{X}^{g p}$-indexed algebra $\tilde{\mathcal{D}}_{X^{(m)} / S}^{(0)}$}

We start with a general theory of $\log \mathcal{D}$-modules of higher level. For details in more general settings, see Chapitre 3 of [9]. Let $X \rightarrow S$ be a log smooth morphism of fine log schemes defined over $Z_{1} / p Z$. We consider the following commutative diagram:

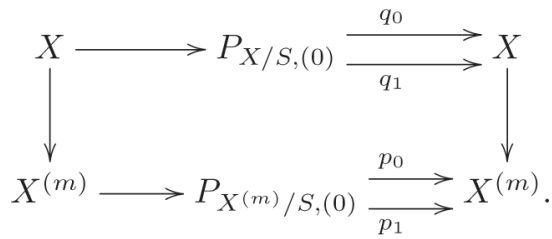

Here the vertical two arrows are the $m$-th relative Frobenius of $X \rightarrow S$ defined in Subsection 3.2 and $q_{0}$ and $q_{1}$ (resp. $p_{0}$ and $p_{1}$ ) are the first and second projections. We denote by $F$ the $m$-th relative Frobenius of $X \rightarrow S$ by abuse of notation. Then, by the universal property of log 0-PD envelopes, there exists a unique morphism of 0 -PD fine $\log$ schemes $F_{\triangle}: P_{X / S,(0)} \rightarrow P_{X^{(m)} / S,(0)}$ which fits into the above diagram.

Proposition 4.5. Let $X \rightarrow S$ be a log smooth morphism of fine log schemes.

(1) There exists $\Phi: P_{X / S,(m)} \rightarrow P_{X^{(m)} / S,(0)}$ such that $F_{\triangle}$ uniquely factors as $P_{X / S,(0)} \rightarrow P_{X / S,(m)} \stackrel{\Phi}{\rightarrow} P_{X^{(m)} / S}$, where the first map is the natural homomorphism.

(2) Assume that we are given a logarithmic system of coordinates $\left\{m_{i}\right\}$ of $X \rightarrow S$. Let $\left\{\underline{\eta}^{\{\underline{k}\}(m)}\right\}$ (resp. $\left\{\underline{\eta}^{\prime \underline{k}\}(0)}\right\}$ ) denote a basis of $\mathcal{P}_{X / S,(m)}$ (resp. $\left.\mathcal{P}_{X^{(m)} / S,(0)}\right)$ associated to the basis $\left\{d \log m_{i}\right\}$ (resp. $\left\{d \log \pi^{*} m_{i}\right\}$ ) (see Proposition 3.11), where $\pi$ denotes the natural projection $X^{(m)} \rightarrow X$ explained in the beginning of Subsection 3.2. Then $\Phi^{*}: \mathcal{P}_{X^{(m)} / S,(0)} \rightarrow \mathcal{P}_{X / S,(m)}$ sends $\underline{\eta}^{\prime\{\underline{k}\}(0)}$ to $\underline{\eta}^{\left\{p^{m} \underline{k}\right\}(m)}$.

Proof. (1) see Proposition 3.3.1 of [9]. (2) See (i) of Proposition 3.4.1 of [9].

Let $\mathcal{E}$ be a left $\mathcal{D}_{X^{(m)} / S}^{(0)}$-module and $\left\{\varepsilon_{n}\right\}$ the log 0 -stratification on $\mathcal{E}$ via the equivalence in Remark 3.5. By endowing $F^{*} \mathcal{E}$ with a left $\mathcal{D}_{X / S}^{(m)}$-module structure by pulling back $\left\{\varepsilon_{n}\right\}$ via $\Phi$, we have a functor

(5) $\quad F^{*}:\left(\right.$ left $\mathcal{D}_{X^{(m)} / S}^{(0)}$-modules on $\left.X^{(m)}\right) \rightarrow\left(\right.$ left $\mathcal{D}_{X / S}^{(m)}$-modules on $\left.X\right)$.

REMARK 4.6. Let $\mathcal{E}$ be a left $\mathcal{D}_{X^{(m)} / S}^{(0)}$-module. Let $\left\{\underline{\eta}^{\{\underline{k}\}(m)}\right\}$ and $\left\{\underline{\eta}^{\prime\{\underline{k}\}(0)}\right\}$ be as in Proposition 4.5 (2). Let $\left\{\underline{\partial}_{\langle\underline{k}\rangle}\right\}$ (resp. $\left\{\underline{\partial}_{\langle\underline{k}\rangle}^{\prime}\right\}$ ) denote the dual of $\left\{\underline{\eta}^{\{\underline{k}\}(m)}\right\}$ (resp. $\left.\left\{\underline{\eta}^{\prime\{\underline{k}\}(0)}\right\}\right)$. Then the $\mathcal{D}_{X / S}^{(m)}$-action on $F^{*} \mathcal{E}$ is characterized by the following for- 
mula:

$$
\underline{\partial}_{\langle\underline{k}\rangle} \cdot(1 \otimes f)=\left\{\begin{array}{cc}
1 \otimes \underline{\partial}_{\left\langle p^{-m} \underline{k}\right\rangle}^{\prime} \cdot f & \text { if } p^{m} \text { divides } \underline{k} \\
0 & \text { otherwise. }
\end{array}\right.
$$

For the proof of this formula, see Proposition 3.4.1 of [9].

In particular, we can consider $F^{*} \mathcal{D}_{X^{(m)} / S}^{(0)}$ as a left $\mathcal{D}_{X / S}^{(m)}$-module. Now, we define the subalgebra $\mathcal{B}_{X / S}^{(m)}$ of $\mathcal{A}_{X}^{g p}$.

Proposition-Definition 4.7. Let $\mathcal{B}_{X / S}^{(m)}$ be the $\mathcal{I}_{X}^{g p} \cong \mathcal{H o m}_{*}\left(*, \mathcal{I}_{X}^{g p}\right)$-indexed sheaf of abelian groups $\mathcal{B}_{X / S}^{(m)}=\mathcal{H o m}_{\mathcal{D}_{X / S}^{(m)}}\left(F^{*} \mathcal{D}_{X^{(m)} / S}^{(0)}, \mathcal{A}_{X}^{g p}\right)$. We give $\mathcal{O}_{X^{(m)}}$-action on $\mathcal{B}_{X / S}^{(m)}$ by right multiplication of $\mathcal{O}_{X^{(m)}}$ on $F^{*} \mathcal{D}_{X^{(m)} / S}^{(0)}$ and define the morphism $\psi: \mathcal{B}_{X}^{(m)} \rightarrow \mathcal{A}_{X}^{g p}$ by $g \mapsto g(1 \otimes 1)$. Then $\psi$ is injective and $\mathcal{B}_{X / S}^{(m)}$ forms an $\mathcal{I}_{X}^{g p}$-indexed sub $\mathcal{O}_{X^{(m)}}$-algebra of $\mathcal{A}_{X}^{g p}$ induced from the multiplication on $\mathcal{A}_{X}^{g p}$.

Proof. See Lemme 4.2.1 of [9].

REMARK 4.8. In [9], Montagnon defines $\mathcal{B}_{X / S}^{(m)}$ by $\mathcal{H o m}_{\mathcal{D}_{X / S, \mathcal{I}}^{(m)}}\left(F^{*} \mathcal{D}_{X^{(m)} / S, \mathcal{I}}^{(0)}, \mathcal{A}_{X}^{g p}\right)$. It seems to us that a natural definition of $\mathcal{B}_{X / S}^{(m)}$ is $\mathcal{H} o m_{\mathcal{D}_{X / S}^{(m)}}\left(F^{*} \mathcal{D}_{X^{(m)} / S}^{(0)}, \mathcal{A}_{X}^{g p}\right)$ because of the same reason as in Remark 4.3.

Proposition-Definition 4.9. Let $X \rightarrow S$ be a log smooth morphism of fine log schemes. Let $\tilde{\mathcal{D}}_{X^{(m)} / S}^{(0)}$ denote the $\mathcal{I}_{X}^{g p}$-indexed $\mathcal{O}_{X^{(m)}}$-module $\mathcal{B}_{X / S}^{(m)} \otimes_{\mathcal{O}_{X^{(m)}}} \mathcal{D}_{X^{(m)} / S^{(0)}}$. Then there exists a unique $\mathcal{I}_{X}^{g p}$-indexed $\mathcal{O}_{X^{(m)}}$-algebra structure on $\tilde{\mathcal{D}}_{X^{(m)} / S}^{(0)}$ such that the maps $\mathcal{B}_{X / S}^{(m)} \rightarrow \tilde{\mathcal{D}}_{X^{(m)} / S}^{(0)} ; a \mapsto a \otimes 1$ and $\mathcal{D}_{X^{m} / S}^{(0)} \rightarrow \tilde{\mathcal{D}}_{X^{(m)} / S}^{(0)} ; P \mapsto 1 \otimes P$ are homomorphisms and that for any $a \in \mathcal{B}_{X / S}^{(m)}, P \in \mathcal{D}_{X^{(m)} / S}^{(0)}$ and $k \in \mathbb{N}^{r}$, we have the relations $a \otimes P=(a \otimes 1)(1 \otimes P)$ and

$$
\left.\left(1 \otimes \underline{\partial}_{\langle\underline{k}\rangle}^{\prime}\right\rangle\right)(a \otimes 1)=\sum_{\underline{i} \leq \underline{k}}\left(\frac{\underline{k}}{i}\right)\left(\underline{\partial}_{\langle\underline{k}-\underline{i}\rangle}^{\prime} \cdot a\right) \otimes \underline{\partial}_{\langle\underline{i}\rangle}^{\prime} .
$$

Here the notation $\left\{\underline{\partial}_{\langle\underline{k}\rangle}^{\prime}\right\}$ is as in Remark 4.6 .

Proof. See Subsection 4.2.1 of [9].

REMARK 4.10. In general, $\mathcal{B}_{X / S}^{(m)}$ does not coincide with $\mathcal{A}_{X^{(m)}}^{g p}$. For counter-example, see 1.8 of [5].

Finally, we give the local description of $\mathcal{B}_{X / S}^{(m)}$. Let $\left\{\underline{\partial}_{\langle\underline{k}\rangle(m)}\right\}$ be as in Remark 4.6. 
Proposition 4.11. Locally, $\mathcal{B}_{X / S}^{(m)}$ can be written as follows:

$$
\left\{a \in \mathcal{A}_{X}^{g p} \mid \underline{\partial}_{\left\langle p^{s} \underline{\varepsilon}_{i}\right\rangle(m)} a=0 \text { for all } 0 \leq s \leq m-1,1 \leq i \leq r\right\} .
$$

Proof. See Subsection 4.2.1 of [9].

Remark 4.12. By virtue of Proposition 4.11 combined with Proposition 3.7 (4), we can locally write

$$
\mathcal{B}_{X / S}^{(m+1)}=\left\{a \in \mathcal{A}_{X}^{g p} \mid \underline{\partial}_{\left\langle p^{s} \underline{\varepsilon}_{i}\right\rangle(m)} a=0 \text { for all } 0 \leq s \leq m, 1 \leq i \leq r\right\} .
$$

Proposition 4.13. $\mathcal{I}_{X}^{g p}$-indexed $\mathcal{O}_{X}$-algebra $\mathcal{A}_{X}^{g p}$ is locally free as an $\mathcal{I}_{X}^{g p}$ indexed $\mathcal{B}_{X / S}^{(m+1)}$-module with local basis $\left\{\underline{\theta}^{j} \mid \underline{j} \in\left[0, p^{m+1}-1\right]^{r}\right\}$.

Proof. See Corollaire 4.2.1 of [9].

Proposition 4.14. $\mathcal{I}_{X}^{g p}$-indexed $\mathcal{O}_{X^{(m)}}$-algebra $\mathcal{B}_{X / S}^{(m)}$ is locally free as an $\mathcal{I}_{X}^{g p}$ indexed $\mathcal{B}_{X / S}^{(m+1)}$-module with local basis $\left\{\underline{\theta}^{p^{m} j}-\underline{j} \in[0, p-1]^{r}\right\}$.

Proof. In this proof we put $M:=\left[0, p^{m+1}-1\right]^{r}$. Since the assertion is étale local on $X$, we may work in a local situation. Note that $\underline{\theta}^{p^{m} j}-\in \mathcal{B}_{X / S}^{(m)}$ by Proposition 4.4. Let $f$ be a local section of $\mathcal{B}_{X / S}^{(m)}$. By Proposition 4.13, we can write $f=\sum_{j \in M} a_{j} \underline{\underline{\theta}} \underline{j}$ with $a_{j} \in \mathcal{B}_{X / S}^{(m+1)}$. Then we calculate

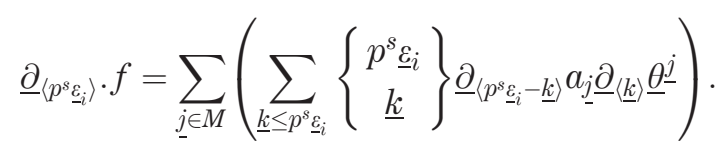

From the proof of Proposition 2.3.1 in [9], $\underline{\partial}_{\left\langle p^{s} \varepsilon_{-}-\underline{k}\right\rangle}$ belongs to the $\mathcal{O}_{X}$-subalgebra of $\mathcal{D}_{X / S}^{(m)}$ generated by $\left\{\underline{\partial}_{\left\langle p^{s} \underline{\varepsilon}_{i}\right\rangle} \mid 0 \leq i \leq m-1\right\}$. So $\underline{\partial}_{\left\langle p^{s} \underline{\varepsilon}_{i}-\underline{k}\right\rangle} a_{j}=0$ if $\underline{k} \neq p^{s} \underline{\varepsilon}_{i}$. Hence, by Proposition 4.4, we have

$$
\underline{\partial}_{\left\langle p^{s} \underline{\varepsilon}_{i}\right\rangle} \cdot f=\sum_{j \in M} a_{j}\left(\begin{array}{c}
j_{i} \\
p^{s}
\end{array}\right) \underline{\theta}^{j}=0 \quad \text { for any } 1 \leq s \leq m-1, \text { and } 1 \leq i \leq r .
$$

Let us calculate the $p$-adic valuation of $\left(\begin{array}{l}j_{i} \\ p^{s}\end{array}\right)$. Let $v$ be the normalized $p$-adic valuation. In the case that $p^{m}$ divides $j_{i}$, we easily calculate $v\left(\left(\begin{array}{l}j_{i} \\ p^{s}\end{array}\right)\right)>0$ for any $1 \leq s \leq m-1$. In the case that $p^{m}$ does not divide $j_{i}$, if we write $j_{i}=\sum b_{l} p^{l}$, then there exists $1 \leq s \leq m-1$ such that $b_{s}>0$. We obtain $v\left(\left(\begin{array}{l}j_{i} \\ p^{s}\end{array}\right)\right)=0$ for this $s$. Since $\left\{\underline{\theta}^{j} \mid \underline{j} \in M\right\}$ is linearly independent, we conclude that we can only have $a_{j} \neq 0$ if $p^{m}$ divides $j$. We finish the proof. 


\section{2 - The Azumaya algebra property}

We first prove the Azumaya algebra property of $\tilde{\mathcal{D}}_{X / S}^{(m)}$ over its center. To do this, first we calculate the center of $\tilde{\mathcal{D}}_{X / S}^{(m)}$. Throughout this subsection we assume that all $\log$ schemes are defined over $Z / p Z$.

Let $X \rightarrow S$ be a $\log$ smooth morphism of fine $\log$ schemes and $F_{X / S}: X \rightarrow X^{\prime}$ the relative Frobenius of $X \rightarrow S$ defined in Subsection 3.2. We have constructed the $p^{m+1}$-curvature map $\beta: \mathcal{T}_{X^{\prime} / S} \rightarrow F_{X / S *} \mathcal{D}_{X}^{(m)}$ (Definition 3.10).

LEMMA 4.15. The image of $\mathcal{T}_{X^{\prime} / S}$ under the $p^{m+1}$-curvature map $\beta$ is contained in the center of $\mathcal{D}_{X / S}^{(m)}$.

Proof. Since the assertion is étale local on $X$, we may work with the local description of $\beta$ (Proposition 3.11). Since $\mathcal{D}_{X / S}^{(m)}$ is generated by $\left\{\underline{\partial}_{\left\langle p^{s} \underline{\varepsilon}_{i}\right\rangle} \mid 1 \leq i \leq r, 1 \leq s \leq m\right\}$ as an $\mathcal{O}_{X}$-algebra, we need to calculate $\underline{\partial}_{\left\langle p^{m+1} \underline{\varepsilon}_{j}\right\rangle} \cdot a$ and $\underline{\partial}_{\left\langle p^{m+1} \underline{\varepsilon}_{j}\right\rangle} \cdot \underline{\partial}_{\left\langle p^{s} \underline{\varepsilon}_{i}\right\rangle}$ for any $a \in \mathcal{O}_{X}, 1 \leq i, j \leq r$ and $1 \leq s \leq m$. By Proposition 3.7 (2), we obtain $\left.\underline{\partial}_{\left\langle p^{m+1} \underline{\varepsilon}_{j}\right\rangle} \cdot \underline{\partial}_{\left\langle p^{s} \underline{\varepsilon}_{i}\right\rangle}=\underline{\partial}_{\left\langle p^{s} \underline{\varepsilon}_{i}\right\rangle} \cdot \underline{\partial}_{\left\langle p^{m+1} \underline{\varepsilon}_{j}\right\rangle}\right\rangle$ Note that, by Proposition 3.7 (4), we have $\underline{\partial}_{\left\langle p^{m+1} \underline{\varepsilon}_{j}\right\rangle(m)}(a)=p ! \underline{\partial}_{\left\langle p^{m+1} \underline{\varepsilon}_{j}\right\rangle(m+1)}(a)=0$. Therefore, by Proposition 3.7 (3), we also obtain

$$
\begin{aligned}
\underline{\partial}_{\left\langle p^{m+1} \underline{\varepsilon}_{j}\right\rangle} \cdot a & \left.=\sum_{i \leq p^{m+1}}\left\{\begin{array}{c}
p^{m+1} \\
i
\end{array}\right\} \underline{\partial}_{\left\langle\left(p^{m+1}-i\right) \underline{\varepsilon}_{j}\right\rangle}(a) \underline{\partial}_{\left\langle\underline{\varepsilon}_{j}\right\rangle}\right\rangle \\
& =a \cdot \underline{\partial}_{\left\langle p^{m+1} \underline{\varepsilon}_{j}\right\rangle} .
\end{aligned}
$$

We finish the proof.

Hence we obtain a morphism of rings

$$
S \cdot \mathcal{T}_{X^{\prime} / S} \rightarrow F_{X / S *} \mathcal{D}_{X / S}^{(m)}
$$

We also denote this map by $\beta$. Let us put $\oslash:=S \cdot \mathcal{T}_{X^{\prime} / S}$ and regard $\oslash$ as a subring of $\mathcal{D}_{X / S}^{(m)}$ via the map $\beta$. The following theorem is due to Schepler when $m$ is equal to zero (see Theorem 2.14 of [11]).

THEOREM 4.16. Let $X \rightarrow S$ be a log smooth morphism of fine log schemes. Let $\tilde{乃}$ denote the center of $\tilde{\mathcal{D}}_{X / S}^{(m)}$. Then $\tilde{\bigcirc}$ is isomorphic to $\mathcal{B}_{X / S}^{(m+1)} \otimes_{\mathcal{O}_{X^{\prime}}} 8$ as an indexed subalgebra of $\tilde{\mathcal{D}}_{X / S}^{(m)}$.

Proof. We may consider the question étale locally on $X$. Let $m_{1}, \ldots, m_{r} \in \mathcal{M}_{X}$ be a logarithmic system of coordinates. If $f \in \mathcal{B}_{X / S}^{(m+1)}$, then, since $\underline{\partial}_{\left\langle p^{s} \underline{\varepsilon}_{i}\right\rangle} \cdot f=0$ for 
any $0 \leq s \leq m, 1 \leq i \leq r$ (Remark 4.11), we have

$$
\begin{aligned}
\left(1 \otimes \underline{\partial}_{\left\langle p^{s} \underline{\varepsilon}_{i}\right\rangle}\right)(f \otimes 1) & =\sum_{j \leq p^{s}}\left\{\begin{array}{c}
p^{s} \\
j
\end{array}\right\}\left(\underline{\partial}_{\left\langle\left(p^{s}-j\right) \underline{\varepsilon}_{i}\right\rangle} \cdot f\right) \otimes \underline{\partial}_{\left\langle j \underline{\varepsilon}_{i}\right\rangle} \\
& =f \otimes \underline{\partial}_{\left\langle p^{s} \underline{\varepsilon}_{i}\right\rangle} \\
& =(f \otimes 1)\left(1 \otimes \underline{\partial}_{\left\langle p^{s} \underline{\varepsilon}_{i}\right\rangle}\right)
\end{aligned}
$$

for any $0 \leq s \leq m, 1 \leq i \leq r$. Since $\left\{\underline{\partial}_{\left\langle p^{s} \underline{\varepsilon}_{i}\right\rangle} \mid 0 \leq s \leq m, 1 \leq i \leq r\right\}$ generate $\mathcal{D}_{X / S}^{(m)}$ (Proposition 3.7 ), we see that $f$ commutes with $\mathcal{D}_{X / S}^{(m)}$ and it obviously commutes with $\mathcal{A}_{X}^{g p}$. Hence we have $f \in \tilde{\Xi}$ and see that $\mathcal{B}_{X / S}^{(m+1)} \otimes_{\mathcal{O}_{X^{\prime}}} \oslash \subset \tilde{g}$. Conversely, since $\mathcal{A}_{X}^{g p}$ is locally free as an $\mathcal{I}_{X}^{g p}$-indexed $\mathcal{B}_{X / S}^{(m+1)}$-module with local basis $\left\{\underline{\theta}^{i} \mid \underline{i} \in\left[0, p^{m+1}-1\right]^{r}\right\}$ (see Proposition 4.13), $\tilde{\mathcal{D}}_{X / S}^{(m)}$ is generated as a $\mathcal{B}_{X / S}^{(m+1)}$-algebra by $\left\{\theta_{i}, \underline{\partial}_{\left\langle p^{s} \underline{\varepsilon}_{i}\right\rangle} \mid 0 \leq s \leq m, 1 \leq i \leq r\right\}$. First, for a local section $\varphi=\sum_{\underline{k}} f_{\underline{k}} \otimes \underline{\partial}_{\langle\underline{k}\rangle}$ of $\tilde{\mathcal{D}}_{X / S}^{(m)}$, we now calculate commutators $\left[\varphi, \theta_{i}\right]$ and $\left[\varphi, \underline{\partial}_{\left\langle p^{s} \underline{\varepsilon}_{i}\right\rangle}\right]$.

$$
\begin{aligned}
{\left[\varphi, \theta_{i}\right] } & =\sum_{\underline{k}}\left(f_{\underline{k}} \otimes \underline{\partial}_{\langle\underline{k}\rangle}\right) \cdot \theta_{i}-\sum_{\underline{k}} \theta_{i} f_{\underline{k}} \otimes \underline{\partial}_{\langle\underline{k}\rangle} \\
& =\sum_{\underline{k}} f_{\underline{k}}\left(\sum_{\underline{j}\langle\underline{k}}\left\{\begin{array}{l}
\underline{k} \\
j
\end{array}\right\}\left(\underline{\partial}_{\underline{\underline{k}}-\underline{j}\rangle} \cdot \theta_{i}\right) \otimes \underline{\partial}_{\langle\underline{j}\rangle}\right) .
\end{aligned}
$$

By Proposition 4.4, we have

$$
\left[\varphi, \theta_{i}\right]=\sum_{\underline{k}}\left\{\begin{array}{c}
k_{i} \\
k_{i}-1
\end{array}\right\} f_{\underline{k}} \theta_{i} \otimes \underline{\partial}_{\langle\underline{k}\rangle} .
$$

Similarly,

$$
\begin{aligned}
{\left[\varphi, \underline{\partial}_{\left\langle p^{s} \underline{\varepsilon}_{i}\right\rangle}\right] } & \left.=\sum_{\underline{k}} f_{\underline{k}} \otimes \underline{\partial}_{\langle\underline{k}\rangle} \underline{\partial}_{\left\langle p^{s} \underline{\varepsilon}_{i}\right\rangle}-\sum_{\underline{k}} \sum_{0 \leq l \leq p^{s}}\left\{\begin{array}{c}
p^{s} \underline{\varepsilon}_{i} \\
l \underline{\varepsilon}_{i}
\end{array}\right\} \underline{\partial}_{\left\langle\left(p^{s}-l\right) \underline{\varepsilon}_{i}\right\rangle} \cdot f_{\underline{k}} \otimes \underline{\partial}_{\left\langle\underline{\varepsilon}_{i}\right\rangle} \underline{\partial}_{\langle\underline{k}\rangle}\right\rangle \\
& =-\sum_{\underline{k}} \sum_{0 \leq l<p^{s}}\left\{\begin{array}{c}
p^{s} \underline{\varepsilon}_{i} \\
l \underline{\varepsilon}_{i}
\end{array}\right\} \underline{\partial}_{\left\langle\left(p^{s}-l\right) \underline{\varepsilon}_{i}\right\rangle} \cdot f_{\underline{k}} \otimes \underline{\partial}_{\left\langle\underline{\varepsilon}_{i}\right\rangle} \underline{\partial}_{\langle\underline{k}\rangle} \\
& =-\sum_{\underline{k}} \sum_{0 \leq l<p^{s}} \underline{\partial}_{\left\langle\left(p^{s}-l\right) \underline{\varepsilon}_{i}\right\rangle} \cdot f_{\underline{k}} \otimes \underline{\partial}_{\left\langle\underline{\varepsilon}_{i}\right\rangle} \underline{\partial}_{\langle\underline{k}\rangle} \cdot
\end{aligned}
$$

Note that $\left\{\begin{array}{c}k_{i} \\ k_{i}-1\end{array}\right\}=0$ in characteristic $p$ if and only if $p^{m+1} \mid k_{i}$. By (7), if $\varphi \in \tilde{3}$ then $f_{\underline{k}} \neq 0$ only for $\underline{k}$ with $p^{m+1} \mid \underline{k}$. Hence, by (8) and Lemma 3.8, we have $\left.\underline{\partial}_{\left\langle p^{s} \underline{\varepsilon}_{i}\right\rangle}\right\rangle \bar{f}_{\underline{k}}=0$ for any $0<s \leq m, 1 \leq i \leq r$, that is, $f_{\underline{k}} \in \mathcal{B}_{X / S}^{(m+1)}$. Since the $p^{m+1}$-curvature map sends $\xi_{i}^{\prime}$ to $\underline{\partial}_{\left\langle p^{m+1} \underline{\varepsilon}_{i}\right\rangle}$, its image is generated as an $\mathcal{O}_{X^{\prime}}$-algebra by $\left\{\underline{\partial}_{\left\langle p^{m+1} \underline{k}\right\rangle} \mid \underline{k} \in \mathbb{N}^{r}\right\}$ by Lemma 3.8. Therefore $\varphi \in \tilde{\mathrm{O}}$ implies $\varphi \in \mathcal{B}_{X / S}^{(m+1)} \otimes_{\mathcal{O}_{X^{\prime}}}$ 8. This completes the proof. 
We also calculate the centralizer of $\mathcal{A}_{X}^{g p}$ in $\tilde{\mathcal{D}}_{X / S}^{(m)}$ in a similar manner. By the calculation (7), we also see the following.

Proposition 4.17. Let $\mathcal{C}_{X}$ denotes the centralizer of $\mathcal{A}_{X}^{g p}$ in $\tilde{\mathcal{D}}_{X / S}^{(m)}$. Then $\mathcal{C}_{X}=$ $\mathcal{A}_{X}^{g p} \otimes_{\mathcal{O}_{X}} \bigcirc$ as an indexed subalgebra of $\tilde{\mathcal{D}}_{X / S}^{(m)}$.

Let us consider $\tilde{\mathcal{D}}_{X / S}^{(m)}$ as a right $\mathcal{C}_{X}$-module by multiplication on the right.

THEOREM 4.18. Let $X \rightarrow S$ be a log smooth morphism offine log schemes. Then there is an isomorphism of $\mathcal{I}_{X}^{g p}$-indexed $\mathcal{C}_{X}$-algebras

$$
\begin{aligned}
& \tilde{\mathcal{D}}_{X / S}^{(m)} \otimes_{\tilde{j}} \mathcal{C}_{X} \longrightarrow \mathcal{E} n d_{\mathcal{C}_{X}}\left(\tilde{\mathcal{D}}_{X / S}^{(m)}\right) \\
& \varphi \otimes c \quad \longmapsto \quad\{x \mapsto \varphi \cdot x \cdot c\} .
\end{aligned}
$$

REMARK 4.19. Theorem 4.18 is due to Schepler in the case $m=0$ (see Theorem 2.15 of [11]) and is due to Gros, Le Stum and Quirós in the case when $X \rightarrow S$ is a smooth morphism of schemes (see Theorem 3.7 of [4]).

Proof. Since the assertion is étale local on $X$, we may assume that there is a logarithmic system of coordinates $m_{1}, \ldots, m_{r} \in \mathcal{M}_{X}$. Then $\tilde{\mathcal{D}}_{X / S}^{(m)} \otimes_{\tilde{\gamma}} \mathcal{C}_{X}$ has a basis $\left\{1 \otimes \underline{\theta}^{i} \mid \underline{i} \in\left[0, p^{m+1}-1\right]^{r}\right\}$ as a left $\tilde{\mathcal{D}}_{X / S}^{(m)}$-module. We set

$$
\beta_{i}=\theta_{i}^{-1} \otimes \theta_{i}-1 \otimes 1 \text {. }
$$

Note that this sum is well-defined since $\theta_{i}^{-1} \otimes \theta_{i}$ and $1 \otimes 1$ are both in the fiber of $\tilde{\mathcal{D}}_{X / S}^{(m)} \otimes_{\tilde{j}} \mathcal{C}_{X}$ at 0 . We put $\underline{\beta}_{-}^{j}:=\prod_{i=1}^{r} \beta_{i}^{j_{i}}$ for $\underline{j} \in\left[0, p^{m+1}-1\right]^{r}$. By the binomial theorem, we have

$$
\underline{\beta}^{j}=\sum_{\underline{i} \leq \underline{j}}\left((-1)^{|\underline{j}-\underline{i}|}\left(\frac{j}{\underline{j}}\right) \theta^{-\underline{i}}\right)\left(1 \otimes \theta^{\underline{i}}\right) .
$$

Thus, if we endow $M$ with some suitable order compatible with the product partial order then the transition matrix from the basis $\left\{1 \otimes \underline{\theta}^{\underline{i}} \mid \underline{i} \in\left[0, p^{m+1}-1\right]^{r}\right\}$ to the set $\left\{\underline{\beta}^{j} \mid \underline{j} \in\left[0, p^{m+1}-1\right]^{r}\right\}$ of $\tilde{\mathcal{D}}_{X / S}^{(m)} \otimes_{\tilde{z}} \mathcal{C}_{X}$ is upper triangular, with units on the diagonal. Therefore $\{\underline{\beta}|\underline{j}| \underline{j} \in M\}$ is also a basis for $\tilde{\mathcal{D}}_{X / S}^{(m)} \otimes_{\tilde{3}} \mathcal{C}_{X}$. On the other hand, let us consider the image of $\left\{\underline{\beta}^{j} \mid \underline{j} \in\left[0, p^{m+1}-1\right]^{r}\right\}$ by the map (9). For $\underline{i} \in M$, let $\alpha_{i}$ be the unique homomorphism which sends $\underline{\partial}_{\langle\underline{j}\rangle}$ to $\delta_{\underline{i j}-}$. Then, since $\tilde{\mathcal{D}}_{X / S}^{(m)}$ has a basis $\left\{\underline{\partial}_{\underline{i}\rangle} \mid \underline{i} \in\left[0, p^{m+1}-1\right]^{r}\right\}$ as a right $\mathcal{C}_{X}$-module, $\left\{\alpha_{\underline{i}} \mid \underline{i} \in\left[0, p^{m+1}-1\right]^{r}\right\}$ forms a basis for $\mathcal{E} n d_{\mathcal{C}_{X}}\left(\tilde{\mathcal{D}}_{X / S}^{(m)}\right)$ as a left $\tilde{\mathcal{D}}_{X / S}^{(m)}$-module. By Proposition 3.7 (3) and 4.4, we now 
calculate that $\beta_{i}$ acts on $\underline{\partial}_{\langle\underline{k}\rangle}$ by

$$
\begin{aligned}
\beta_{i} \cdot \underline{\partial}_{\langle\underline{k}\rangle} & =\theta_{i}^{-1} \cdot \underline{\partial}_{\langle\underline{k}\rangle} \cdot \theta_{i}-\underline{\partial}_{\langle\underline{k}\rangle} \\
& =\theta_{i}^{-1}\left(\theta_{i} \otimes \underline{\partial}_{\langle\underline{k}\rangle}+\left\{\begin{array}{c}
k_{i} \\
k_{i}-1
\end{array}\right\} \theta_{i} \otimes \underline{\partial}_{\left\langle\underline{k}-\underline{\varepsilon}_{i}\right\rangle}\right)-\underline{\partial}_{\langle\underline{k}\rangle} \\
& =\left\{\begin{array}{c}
k_{i} \\
k_{i}-1
\end{array}\right\} \underline{\partial}_{\left\langle\underline{k}-\underline{\varepsilon}_{i}\right\rangle} .
\end{aligned}
$$

Hence $\underline{\beta}_{-}^{j}$ acts on $\tilde{\mathcal{D}}_{X / S}^{(m)}$ by sending $\underline{\partial}_{\langle\underline{k}\rangle}$ to $\frac{\underline{q} \underline{\underline{q}} !}{\underline{\underline{q}-j} !} \underline{\partial}_{\langle\underline{k}-j\rangle}$ if $\underline{k} \geq \underline{j}$ and 0 otherwise. We thus obtain

$$
\underline{\beta} \underline{j}=\sum_{\underline{i} \geq \underline{j}, \underline{i} \in M}\left(\frac{\underline{q}_{\underline{i}} !}{\underline{q}_{\underline{i}-j} !} \underline{\partial}_{\langle\underline{i}-\underline{j}\rangle}\right) \alpha_{\underline{i}}
$$

in $\mathcal{E} n d_{\mathcal{C}_{X}}\left(\tilde{\mathcal{D}}_{X}^{(m)}\right)$ and see the transition matrix from the basis $\left\{\alpha_{\underline{i}} \mid \underline{i} \in\left[0, p^{m+1}-1\right]^{r}\right\}$ to the set $\left\{\underline{\beta} \underline{\underline{j}} \mid \underline{j} \in\left[0, p^{m+1}-1\right]^{r}\right\}$ of $\mathcal{E} n d_{\mathcal{C}_{X}}\left(\tilde{\mathcal{D}}_{X}^{(m)}\right)$ is lower triangular with units on the diagonal. We see that $\left\{\underline{\beta}-\underline{j} \mid \underline{j} \in\left[0, p^{m+1}-1\right]^{r}\right\}$ is also a basis for $\mathcal{E} n d_{\mathcal{C}_{X}}\left(\tilde{\mathcal{D}}_{X}^{(m)}\right)$.

COROLlary. Let $X \rightarrow S$ be a log smooth morphism offine log schemes. The $\mathcal{I}_{X}^{g p}$ indexed $\mathcal{O}_{X}$-algebra $\tilde{\mathcal{D}}_{X / S}^{(m)}$ is an Azumaya algebra over its center $\tilde{8}$ of rank $p^{2(m+1) r}$.

Proof. From Proposition 4.13, $\mathcal{A}_{X}^{g p}$ is locally free as an $\mathcal{I}_{X}^{g p}$-indexed $\mathcal{B}_{X / S}^{(m+1)}$ module. Thus $\mathcal{C}_{X} \cong \mathcal{A}_{X}^{g p} \otimes_{\mathcal{B}_{X S}^{(m+1)}} \tilde{\Im}$ is a faithfully flat extension of $\tilde{\mathfrak{O}}$. By Theorem 4.18, $\tilde{\mathcal{D}}_{X / S}^{(m)}$ splits over $\mathcal{C}_{X}$ with splitting module $\tilde{\mathcal{D}}_{X / S}^{(m)}$.

The proof of the Azumaya nature of $\tilde{\mathcal{D}}_{X^{(m)} / S}^{(0)}$ is the same as that of $\tilde{\mathcal{D}}_{X / S}^{(m)}$ except for obvious modifications.

THEOREM 4.20. Let $X \rightarrow S$ be a log smooth morphism of fine log schemes.

(1) Let $\tilde{\mathfrak{S}}^{\prime}$ denote the center of $\tilde{\mathcal{D}}_{X^{(m)} / S}^{(0)}$. Then the $p$-curvature map $8 \rightarrow \mathcal{D}_{X^{(m)} / S}^{(0)}$ induces an isomorphism between $\mathcal{B}_{X / S}^{(m+1)} \otimes_{\mathcal{O}_{X^{\prime}}} 8$ and $\tilde{\mathcal{D}}^{\prime}$ as an indexed subalgebra of $\tilde{\mathcal{D}}_{X^{(m)} / S}^{(0)}$.

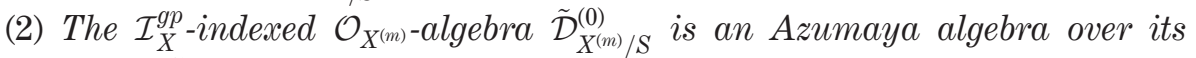
center $\tilde{O}^{\prime}$ of rank $p^{2 r}$.

Proof. We shall only sketch a proof. Take $\varphi \in \tilde{\mathcal{D}}_{X^{(m)} / S}^{(0)}$. As in the proof of Theorem 4.16, we consider the necessary and sufficient condition for $\varphi \in \tilde{\Re}^{\prime}$. Recall that $\mathcal{B}_{X / S}^{(m)}$ is locally free as an $\mathcal{I}_{X}^{g p}$-indexed $\mathcal{B}_{X / S}^{(m+1)}$-module with a local basis $\left\{\underline{\theta}^{p^{m} \underline{j}} \mid \underline{j} \in[0, p-1]^{r}\right\}$ (see Proposition 4.14). Thus $\tilde{\mathcal{D}}_{X^{(m)} / S}^{(0)}$ is generated by 
$\left\{\theta_{i}^{p^{m}}, \underline{\partial}_{\left\langle\underline{\varepsilon}_{i}\right\rangle(0)} \mid 1 \leq i \leq r\right\}$ as an $\mathcal{I}_{X}^{g p}$-indexed $\mathcal{B}_{X / S}^{(m+1)}$-algebra. Let us describe $\varphi=\sum_{\underline{k}} f_{\underline{k}} \otimes \underline{\partial}_{\langle\underline{k}\rangle(0)}$ with $f_{\underline{k}} \in \mathcal{B}_{X / S}^{(m+1)}$. Then commutators $\left[\varphi, \theta_{i}^{p^{m}}\right]$ and $\left[\varphi, \underline{\partial}_{\left.\underline{\varepsilon}_{i}\right\rangle}\right]$ can be calculated as follows.

$$
\begin{aligned}
{\left[\varphi, \theta_{i}^{p^{m}}\right] } & \left.=\sum_{\underline{k}} k_{i} f_{\underline{k}} \theta_{i}^{p^{m}} \otimes \underline{\partial}_{\left\langle\underline{k}-\underline{\varepsilon}_{i}\right\rangle}\right\rangle . \\
{\left[\varphi, \underline{\partial}_{\left\langle\underline{\varepsilon}_{\rangle}\right\rangle}\right] } & =-\sum_{\underline{k}} \underline{\partial}_{\left\langle\underline{\varepsilon}_{i}\right\rangle(0)} f_{\underline{k}} \otimes \underline{\partial}_{\langle\underline{k}\rangle} .
\end{aligned}
$$

By (10), if $\varphi \in \tilde{\mathfrak{Z}}^{\prime}$ then $f_{\underline{k}} \neq 0$ only for $p \mid \underline{k}$. By (11) and (6), we have $\underline{\partial}_{\left\langle p^{m} \underline{\varepsilon}_{\underline{o}}\right\rangle(m)} f_{\underline{\underline{k}}}=$ $\underline{\partial}_{\left\langle\underline{\varepsilon}_{i}\right\rangle(0)} f_{\underline{k}}=0$ for any $1 \leq i \leq r$, that is, $f_{\underline{k}} \in \mathcal{B}_{X / S}^{(m+1)}$. This finishes the proof of (1). For proving (2), let $\mathcal{C}_{X^{(m)}}$ be the centralizer of $\mathcal{B}_{X / S}^{(m)}$ in $\tilde{\mathcal{D}}_{X^{(m)} / S}^{(0)}$ which is isomorphic to $\mathcal{B}_{X / S}^{(m)} \otimes_{\mathcal{O}_{X^{\prime}}} 8^{\prime}$. Then, by an analogous argument to the proof of Theorem 4.18, one can obtain the isomorphism

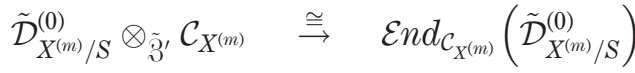

$$
\begin{aligned}
& \varphi \otimes c \quad \longmapsto \quad\{x \mapsto \varphi \cdot x \cdot c\} .
\end{aligned}
$$

Therefore (2) follows from Proposition 4.14.

REMark 4.21. Theorem 4.20 is a variant of Theorem 2.14 and Theorem 2.15 in [11].

\section{3 - The log Cartier descent theorem}

As a first application of Corollary 4.2-, we prove the log Cartier descent theorem of higher level. (See Corollary 3.2.4 of [6] for the case without log structure.)

First we generalize the notion of admissible connection to the case of higher level.

Definition 4.22. Let $\mathcal{J}$ be a sheaf of $\mathcal{I}_{X}^{g p}$-sets on $X$ and let $\mathcal{E}$ be a $\mathcal{J}$-indexed $\mathcal{A}_{X}^{g p}$ module with a $\mathcal{J}$-indexed left $\mathcal{D}_{X / S}^{(m)}$-module structure. Then the action of $\mathcal{D}_{X / S}^{(m)}$ on $\mathcal{E}$ is admissible if the $\mathcal{J}$-indexed $\mathcal{A}_{X}^{g p}$-module structure and the $\mathcal{J}$-indexed left $\mathcal{D}_{X / S^{-}}^{(m)}$ module structure on $\mathcal{E}$ extend to the $\mathcal{J}$-indexed $\tilde{\mathcal{D}}_{X / S}^{(m)}$-module structure on $\mathcal{E}$. We also define the notion of admissibleness for a $\mathcal{J}$-indexed $\mathcal{B}_{X / S}^{(m)}$-module with a $\mathcal{J}$-indexed

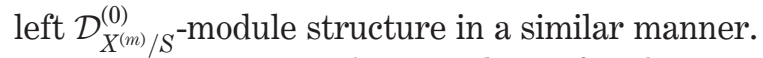

We give an equivalent condition for the action of $\mathcal{D}_{X / S}^{(m)}$ on a $\mathcal{J}$-indexed $\mathcal{A}_{X}^{g p}$ module to be admissible. Let $\mathcal{E}$ be a $\mathcal{J}$-indexed $\mathcal{A}_{X}^{g p}$-module. Recall from Remark 2.4 that the action of $\mathcal{A}_{X}^{g p}$ on $\mathcal{E}$ is equivalent to the family of morphisms $\rho_{i j}$ : $\mathcal{A}_{X, i}^{g p} \otimes_{\mathcal{O}_{U}} \mathcal{E}_{j} \rightarrow \mathcal{E}_{i+j}$ satisfying suitable conditions. 
Proposition 4.23. Let $\mathcal{J}$ be a sheaf of $\mathcal{I}_{X}^{g p}$-sets on $X$ and let $\mathcal{E}$ be a $\mathcal{J}$-indexed $\mathcal{A}_{X}^{g p}$-module with an $\mathcal{J}$-indexed left $\mathcal{D}_{X / S^{(m)}}^{(m)}$-module structure. Then the action of $\mathcal{D}_{X / S}^{(m)}$ on $\mathcal{E}$ is admissible if and only if the corresponding structural morphism $\rho_{i j}: \mathcal{A}_{X, i}^{g p} \otimes_{\mathcal{O}_{U}} \mathcal{E}_{j} \rightarrow \mathcal{E}_{i+j} ; a \otimes e \mapsto$ ae is a $\mathcal{D}_{X / S^{-}}^{(m)}$-homomorphism for any étale open $U$ of $X$ and any section $(i, j) \in \mathcal{I}_{X}^{g p} \times \mathcal{J}(U)$. Here $\mathcal{A}_{X, i}^{g p} \otimes_{\mathcal{O}_{U}} \mathcal{E}_{j}$ is a tensor product as a left $\mathcal{D}_{X / S}^{(m)}$-module.

PRoOF. We show the if part. Let us show that the action of $\tilde{\mathcal{D}}_{X / S}^{(m)}$ on $\mathcal{E}$ defined by $\tilde{\mathcal{D}}_{X, i}^{(m)} \otimes \mathcal{O}_{U} \mathcal{E}_{j} \rightarrow \mathcal{E}_{i+j}(a \otimes P) \otimes e \mapsto a . P . e$ is well-defined. We may work locally and, by assumption, we have

$$
\underline{\partial}_{\langle\underline{k}\rangle} \cdot(a \cdot e)=\sum_{\underline{i} \leq \underline{k}}\left\{\frac{k}{\underline{k}}\right\} \underline{\partial}_{\langle\underline{k}-\underline{i}\rangle} \cdot a \underline{\partial}_{\underline{\langle} \underline{\underline{i}}} \cdot e .
$$

On the other hand, we have

$$
\begin{aligned}
\left(\underline{\partial}_{\langle\underline{k}\rangle} \cdot a\right) \cdot e & =\left(\sum_{\underline{i} \leq \underline{k}}\left\{\begin{array}{l}
\underline{k} \\
\underline{i}
\end{array}\right\} \underline{\partial}_{\langle\underline{k}-\underline{i}\rangle} \cdot a \otimes \underline{\partial}_{\langle\underline{i}\rangle}\right) \cdot e \\
& =\sum_{\underline{i} \leq \underline{k}}\left\{\begin{array}{c}
\underline{k} \\
\underline{i}
\end{array}\right\} \underline{\partial}_{\langle\underline{k}-\underline{i}\rangle} \cdot a \underline{\partial}_{\langle\underline{i}\rangle} \cdot e .
\end{aligned}
$$

This completes the proof.

REMARK 4.24. We can also give a condition for the action of $\mathcal{D}_{X / S}^{(m)}$ on a $\mathcal{J}$-indexed $\mathcal{A}_{X}^{g p}$-module to be admissible by using the notion of $\log m$-stratification. Let $p_{i}^{n}$ (with $i=0,1$ ) be the natural projection $P_{X / S,(m)}^{n} \rightarrow X$ and $\left\{\varepsilon_{\mathcal{A}, n}\right\}$ (resp. $\left\{\varepsilon_{\mathcal{E}, n}\right\}$ the $\log m$-stratification on $\mathcal{A}_{X}^{g p}$ (resp. on $\mathcal{E}$ ) associated to the $\mathcal{D}_{X / S^{-}}^{(m)}$ action. Let $\mathcal{E}$ be a $\mathcal{J}$-indexed $\mathcal{A}_{X}^{g p}$-module with a $\mathcal{J}$-indexed left $\mathcal{D}_{X / S}^{(m)}$-module structure. Then a $\mathcal{J}$ indexed left $\mathcal{D}_{X / S}^{(m)}$-module structure on $\mathcal{E}$ is admissible if and only if the following diagram is commutative for any positive integer $n$ :

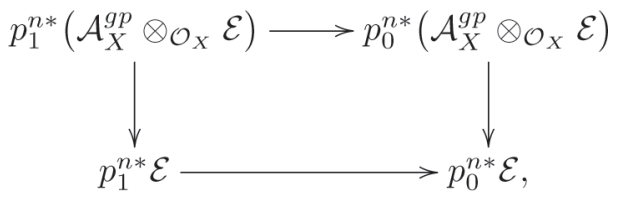

where the upper horizontal arrow is the tensor product $\varepsilon_{\mathcal{A}, n} \otimes \varepsilon_{\mathcal{E}, n}$, the lower horizontal arrow is $\varepsilon_{\mathcal{E}, n}$ and the vertical arrows are induced from the $\mathcal{A}_{X}^{g p}$-action on $\mathcal{E}$.

REMARK 4.25. Let $\mathcal{E}$ be a $\mathcal{D}_{X / S}^{(m)}$-module. Then the $\mathcal{D}_{X / S}^{(m)}$-action on the tensor product $\mathcal{A}_{X}^{g p} \otimes_{\mathcal{O}_{X}} \mathcal{E}$ as a $\mathcal{D}_{X / S^{(m)}}^{(m)}$-module is admissible.

For a $\mathcal{J}$-indexed $\tilde{\mathcal{D}}_{X / S}^{(m)}$-module $\mathcal{E}$, we put $\mathcal{E}^{\nabla}:=\mathcal{H}_{\mathcal{H}^{(m)}}\left(\mathcal{O}_{X}, \mathcal{E}\right)$. 
THeOREm 4.26. Let $X \rightarrow S$ be a log smooth morphism of fine log schemes. Let $\mathcal{J}$ be a sheaf of $\mathcal{I}_{X}^{g p}$-sets on $X$. Then the functor $\mathcal{E} \mapsto \mathcal{E}^{\nabla}$ give an equivalence between the category of $\mathcal{J}$-indexed $\mathcal{A}_{X}^{g p}$-modules with an admissible $\mathcal{J}$-indexed left $\mathcal{D}_{X / S^{-}}^{(m)}$ module structure and zero $p^{m+1}$-curvature map and the category of $\mathcal{J}$-indexed $\mathcal{B}_{X / S}^{(m+1)}$-modules.

Proof. We use Proposition 2.7 to construct the equivalence. We consider $\mathcal{B}_{X / S}^{(m+1)}$ as a $\tilde{3}$-algebra via the composite $S\left(\mathcal{T}_{X^{\prime} / S}\right) \rightarrow S\left(\mathcal{T}_{X^{\prime} / S}\right) / S^{+}\left(\mathcal{T}_{X^{\prime} / S}\right) \stackrel{\cong}{\rightrightarrows} \mathcal{O}_{X^{\prime}} \rightarrow \mathcal{B}_{X / S}^{(m+1)}$. By Corollary 4.2-, $\tilde{\mathcal{D}}_{0}:=\tilde{\mathcal{D}}_{X}^{(m)} \otimes_{\tilde{3}} \mathcal{B}_{X / S}^{(m+1)}$ is an Azumaya algebra of rank $p^{2(m+1) r}$. Let us find the splitting module for $\tilde{\mathcal{D}}_{0}$. Since $\mathcal{A}_{X}^{g p}$ is a locally free $\mathcal{B}_{X / S}^{(m+1)}$-module of rank $p^{(m+1) r}$ which has a structure of left $\tilde{\mathcal{D}}_{0}$-module, $\tilde{\mathcal{D}}_{0}$ splits over $\mathcal{B}_{X / S}^{(m+1)}$ with splitting module $\mathcal{A}_{X}^{g p}$ by Proposition 2.9. Hence, we can apply Proposition 2.7 and get the equivalence of categories $\mathcal{E} \mapsto \mathcal{H}_{0} m_{\tilde{\mathcal{D}}_{0}}\left(\mathcal{A}_{X}^{g p}, \mathcal{E}\right)$ between the category of $\mathcal{J}$-indexed left $\tilde{\mathcal{D}}_{0}$-modules and the category of $\mathcal{J}$-indexed $\mathcal{B}_{X / S}^{(m+1)}$-modules. Now the notion of $\mathcal{J}$ indexed left $\tilde{\mathcal{D}}_{0}$-module is equivalent to that of $\mathcal{J}$-indexed $\mathcal{A}_{X}^{g p}$-module with an admissible $\mathcal{J}$-indexed left $\mathcal{D}_{X / S^{\prime m}}^{(m)}$-module structure and zero $p^{m+1}$-curvature, and there is a natural isomorphism $\mathcal{H}_{\tilde{\mathcal{D}}_{0}}\left(\mathcal{A}_{X}^{g p}, \mathcal{E}\right) \cong \mathcal{E}^{\nabla}$. This completes the proof.

\section{The global Cartier transform}

The goal of this section is to construct the log global Cartier transform of higher level. First we recall a few notions on the log crystalline theory of higher level needed later. It should be remarked here that Miyatani studied the foundations of log crystalline theory of higher level in his unpublished master thesis in the University of Tokyo [8].

\section{1 - Logarithmic crystalline site of level $m$}

We fix throughout this subsection an $m$-PD fine $\log$ scheme $(S, \mathfrak{a}, \mathfrak{b}, \gamma)$ and a fine $\log$ scheme $X$ over $S$. We assume that the $m$-PD structure $\gamma$ extends to $X$.

Definition 5.1. Let $U$ be a fine log scheme over $X$. A log $m$-PD thickening $(U, T, J, \delta)$ of $U$ over $(S, \mathfrak{a}, \mathfrak{b}, \gamma)$ is a data which consists of a fine $\log$ scheme $T$ over $S$, an exact closed immersion $U \hookrightarrow T$ over $S$ and an $m$-PD structure $(J, \delta)$ on the defining ideal of $U \hookrightarrow T$ compatible with $(\mathfrak{b}, \gamma)$. A morphism of $\log m$-PD thickenings $\operatorname{over}(S, \mathfrak{a}, \mathfrak{b}, \gamma)$ can be defined in an obvious way.

Definition 5.2. (1) The $\log m$-crystalline site $\operatorname{Cris}^{(m)}(X / S)$ is the category of log $m$-PD thickenings $(U, T, J, \delta)$ of an étale open $U$ of $X$ over $(S, \mathfrak{a}, \mathfrak{b}, \gamma)$ endowed with the topology induced by the étale topology on $T$. Its associated topos $(X / S)_{\text {cris }}^{(m)}$ is called the $\log m$-crystalline topos. 
(2) The sheaf of rings defined by

$$
(U, T, J, \delta) \longmapsto \Gamma\left(T, \mathcal{O}_{T}\right)
$$

in the topos $(X / S)_{\text {cris }}^{(m)}$ is called the structure sheaf of the site $\operatorname{Cris}^{(m)}(X / S)$ and we denote it by $\mathcal{O}_{X / S}^{(m)}$.

REMARK 5.3. A sheaf $E$ on $\operatorname{Cris}^{(m)}(X / S)$ is equivalent to the following data: For every log $m$-PD thickening $(U, T, J, \delta)$, an étale sheaf $E_{T}$ on $T$, and for every morphism $u: T_{1} \rightarrow T_{2}$ in $\operatorname{Cris}^{(m)}(X / S)$, a map $\rho_{u}: u^{-1}\left(E_{T_{2}}\right) \rightarrow E_{T_{1}}$, satisfying the cocycle condition such that $\rho_{u}$ is an isomorphism if $u$ is étale.

Next we define the notion of $\log m$-crystal.

Definition 5.4. Let $E$ be an $\mathcal{O}_{X / S}^{(m)}$-module in $(X / S)_{\text {cris }}^{(m)}$. Then, $E$ is called a log $m$ -

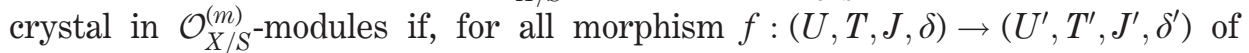
$\operatorname{Cris}^{(m)}(X / S)$, the canonical morphism

$$
f^{*}\left(E_{\left(U^{\prime}, T^{\prime}, J^{\prime}, \delta^{\prime}\right)}\right) \longrightarrow E_{(U, T, J, \delta)}
$$

is an isomorphism.

Definition 5.5. Let $M$ be an $\mathcal{O}_{X}$-module. Then a log hyper $m$-PD stratification on $M$ is a $\mathcal{P}_{X / S,(m)}$-linear isomorphism

$$
\varepsilon: \mathcal{P}_{X / S,(m)} \otimes M \rightarrow M \otimes \mathcal{P}_{X / S,(m)}
$$

which is reduced to the identity map on $M$ modulo the kernel of $\mathcal{P}_{X / S,(m)} \rightarrow \mathcal{O}_{X}$ and satisfies the usual cocycle condition.

Proposition 5.6. Let $\left(\mathfrak{a}_{0}, \mathfrak{b}_{0}, \gamma_{0}\right)$ be a quasi-coherent m-PD subideal of a, let $S_{0} \hookrightarrow S$ denote the exact closed immersion defined by $\mathfrak{a}_{0}$, and $X_{0} \hookrightarrow X$ its base change by $X \rightarrow S$. We assume that $X$ is log smooth and flat over $S$. Then the following categories are equivalent

(1) The category of log m-crystals in $\mathcal{O}_{X_{0} / S^{(m)}}^{(\text {modules }}$

(2) The category of $\mathcal{O}_{X}$-modules equipped with log hyper m-PD stratifications.

Proof. The proof is the same as the classical case. It suffices to see that the log $m$-PD envelope of $X_{0}$ in $X \times_{S} X, X \times_{S} X \times_{S} X$ is equal to the log $m$-PD envelope of $X$ in $X \times_{S} X, X \times_{S} X \times_{S} X$, respectively. This follows from the fact that the $m$-PD structure of the latter is compatible with the $m$-PD structure $\left(\mathfrak{b}_{0}, \gamma_{0}\right)$.

Finally, for technical reasons, we introduce a variant of the big crystalline site.

Definition 5.7. We define a site $\operatorname{CRIS}_{\text {Int }}^{(m)}(X / S)$ as the category of log $m$-PD thickenings $(U, T, J, \delta)$ of $U$ over $S$ such that $U$ is any fine log scheme over $X$ and 
that $T \rightarrow S$ is integral endowed with the topology induced from the étale topology on $T$.

REMARK 5.8. As in the classical case, we obtain an equivalence

$$
\left(\begin{array}{c}
\log m \text {-crystals of } \mathcal{O}_{X / S}^{(m)} \text {-modules } \\
\text { on } \operatorname{Cris}^{(m)}(X / S)
\end{array}\right) \rightarrow\left(\begin{array}{c}
\mathcal{O}_{X} \text {-modules } \\
\text { with } \log m \text {-PD stratifications }
\end{array}\right)
$$

by the following way. Let $\mathcal{E}$ be a $\log m$-crystal of $\mathcal{O}_{X / S}^{(m)}$-modules. Then, for each natural number $n$, the natural morphism $\left(X \hookrightarrow P_{X / S,(m)}^{n}\right) \rightarrow(X \stackrel{\text { id }}{\rightarrow} X)$ in $\operatorname{Cris}^{(m)}(X / S)$ defines an isomorphism of $\mathcal{P}_{X / S,(m)}^{n}$-modules $\varepsilon_{n}: p_{0}^{n *} \mathcal{E}_{X} \cong \mathcal{E}_{P_{X / S,(m)}^{n}} \cong p_{1}^{n *} \mathcal{E}_{X}$. These isomorphisms define a $\log m$-stratification on an $\mathcal{O}_{X}$-module $\mathcal{E}:=\mathcal{E}_{X}$. If $X \rightarrow S$ is $\log$ smooth and integral, a case which interests us in the sequel, then the log $m$-PD envelopes of $X$ in $X \times_{S} X$ and $X \times_{S} X \times_{S} X$ are integral over $S$. So, under these hypotheses, we also obtain an equivalence

$$
\left(\begin{array}{c}
\log m \text {-crystals of } \mathcal{O}_{X / S}^{(m)} \text {-modules } \\
\text { on } \operatorname{CRIS}_{\text {Int }}^{(m)}(X / S)
\end{array}\right) \rightarrow\left(\begin{array}{c}
\mathcal{O}_{X} \text {-modules } \\
\text { with } \log m \text {-PD stratifications }
\end{array}\right)
$$

in a similar manner. Hence the category of $\log m$-crystals on $\operatorname{CRIS}_{\text {Int }}^{(m)}(X / S)$ is equivalent to that on $\mathrm{Cris}^{(m)}(X / S)$ in this case.

REMARK 5.9. Let $(U, T, J, \delta)$ be an object in $\operatorname{CRIS}_{\text {Int }}^{(m)}(X / S)$. Because $U$ and $T$ are integral over $S$ by definition, the underlying scheme of $U^{\prime \prime}=U \times_{S} S$ (resp. $T^{\prime \prime}=T \times{ }_{S} S$ ) coincide with the fiber product of the diagram $U \rightarrow S \leftarrow S$ (resp. $T \rightarrow S \leftarrow S)$ in the category of schemes, where $S \rightarrow S$ is the $(m+1)$-st iterate of its absolute Frobenius $F_{S}$ of $S$. See also Subsection 3.2-.

\section{2 - The global Cartier transform}

Let us set some notations. Let $X \rightarrow S$ be a log smooth morphism of fine $\log$ schemes defined over $Z / p Z$. As in the beginning of Subsection 3.2, we have the following Frobenius diagram:

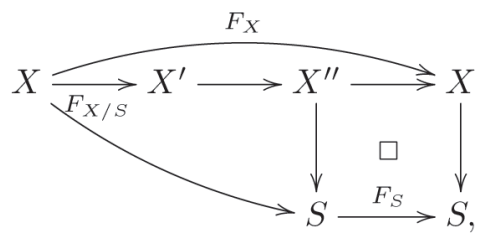

where $F_{S}$ (resp. $\left.F_{X}\right)$ denotes the $(m+1)$-st iterate of the absolute Frobenius of $S$ (resp. $X), X^{\prime \prime}$ is the fiber product in the category of fine log schemes, and $F_{X / S}$ $((m+1)$-st relative Frobenius) is uniquely determined by the requirement that 
$F_{X S}$ is purely inseparable and $X^{\prime} \rightarrow X^{\prime \prime}$ is log étale (see Proposition 4.10 of [5]). We denote the composition $X^{\prime} \rightarrow X^{\prime \prime} \rightarrow X$ by $\pi$. Fix a sheaf of $\mathcal{I}_{X}^{g p}$-sets $\mathcal{J}$ on $X$. Write $\mathcal{G}$ for the nilpotent divided power envelope of the zero section of the cotangent bundle of $X \rightarrow S$, so that $\mathcal{O}_{\mathcal{G}}=\hat{\Gamma} \cdot \mathcal{T}_{X^{\prime} / S}$. We put $\mathcal{O}_{\mathcal{G}}^{\mathcal{B}}:=\mathcal{B}_{X / S}^{(m+1)} \otimes_{\mathcal{O}_{X^{\prime}}} \mathcal{O}_{\mathcal{G}}, \mathcal{O}_{\mathcal{G}}^{\mathcal{A}}:=\mathcal{A}_{X}^{g p} \otimes_{\mathcal{O}_{X^{\prime}}} \mathcal{O}_{\mathcal{G}}$, and $\tilde{\mathcal{D}}_{X / S}^{(m), \gamma}:=\tilde{\mathcal{D}}_{X / S}^{(m)} \otimes_{\tilde{3}} \mathcal{O}_{\mathcal{G}}^{\mathcal{B}}$. We denote by $\operatorname{HIG}_{\mathrm{PD}}^{\mathcal{B}, \mathcal{J}}\left(X^{\prime} / S\right)$ the category of $\mathcal{J}$-indexed $\mathcal{O}_{\mathcal{G}}^{\mathcal{B}}$-modules, and by $\operatorname{MIC}_{\mathrm{PD}}^{\mathcal{A}, \mathcal{J}}(X / S)$ the category of $\mathcal{J}$-indexed $\tilde{\mathcal{D}}_{X / \mathcal{S}}^{(m), \gamma}$-modules. Note that an object of $\operatorname{HIG}_{\mathrm{PD}}^{\mathcal{B}, \mathcal{J}}\left(X^{\prime} / S\right)$ is equivalent to a $\mathcal{J}$-indexed $\mathcal{B}_{X / S}^{(m+1)}$-module $E^{\prime}$ equipped with a homomorphism of indexed $\mathcal{O}_{X^{\prime}}$-algebras

$$
\theta: \mathcal{O}_{\mathcal{G}} \rightarrow \mathcal{E} n d_{\mathcal{B}_{X / S}^{(m+1)}}\left(E^{\prime}\right)
$$

(called a $\mathcal{B}_{X / S}^{(m+1)}$-linear $\mathcal{G}$-Higgs field). Similarly, an object of $\mathrm{MIC}_{\mathrm{PD}}^{\mathcal{A}, \mathcal{J}}(X / S)$ is equivalent to a $\mathcal{J}$-indexed $\mathcal{A}_{X}^{g p}$-module $E$ with an admissible $\mathcal{D}_{X / S}^{(m)}$-action endowed with a homomorphism of indexed algebras

$$
\theta: \mathcal{O}_{\mathcal{G}} \rightarrow F_{X / S *} \mathcal{E} n d_{\tilde{\mathcal{D}}_{X / S}^{(m)}}(E)
$$

extending the map

$$
\psi: S \cdot \mathcal{T}_{X^{\prime} / S} \rightarrow F_{X / S *} \mathcal{E} n d_{\tilde{\mathcal{D}}_{X / S}^{(m)}}(E)
$$

given by the $p^{m+1}$-curvature (called a horizontal $\mathcal{A}_{X}^{g p}$-linear $\mathcal{G}$-Higgs field). The global Cartier transform is formulated as an equivalence of categories between $\operatorname{MIC}_{\mathrm{PD}}^{\mathcal{A}, \mathcal{J}}(X / S)$ and $\operatorname{HIG}_{\mathrm{PD}}^{\mathcal{B}, \mathcal{J}}\left(X^{\prime} / S\right)$. As in [10] and [11], first we study the lifting torsor of the $(m+1)$-st relative Frobenius morphism in the context of crystals.

Definition 5.10. Let $f: Y \rightarrow Z$ be a morphism of fine log schemes defined over $Z / p Z$. Then a lifting of $f$ modulo $p^{n}$ is a morphism $\tilde{f}: \tilde{Y} \rightarrow \tilde{Z}$ of fine log schemes flat over $Z / p^{n} Z$ which fits into a cartesian square in the category of fine log schemes

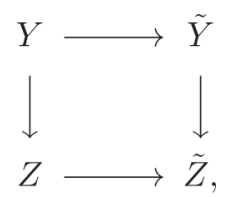

where $Z \rightarrow \tilde{Z}$ is the exact closed immersion defined by $p$.

If $f$ is $\log$ smooth, respectively log étale, resp. integral, resp. exact, so is $\tilde{f}$. From now on, we are mainly concerned with liftings modulo $p^{2}$.

For the rest of this paper, we consider a log smooth integral morphism $f: X \rightarrow S$ of fine $\log$ schemes defined over Z/pZ equipped with a lifting $\tilde{X}^{\prime} \rightarrow \tilde{S}$ of $X^{\prime} \rightarrow S$ modulo $p^{2}$, and we regard $S, \tilde{S}$ as $m$-PD fine $\log$ schemes with the canonical $m$-PD structure on $(p)$. We denote the data $\left(X \rightarrow S, \tilde{X}^{\prime} \rightarrow \tilde{S}\right)$ by $\mathcal{X} / \mathcal{S}$. 
LEMma 5.11. Let $(U, T, J, \delta)$ be an object of $\operatorname{CRIS}_{\text {Int }}^{(m)}(X / S)$. Then there exists $a$ canonical morphism $T \rightarrow U^{\prime}$ such that the following diagram commutes

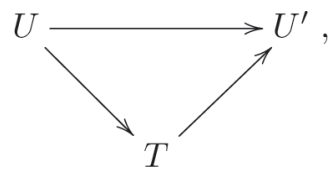

where the symbol' is as in the beginning of Subsection 5.2.

Proof. We use the symbol " as in the beginning of Subsection 5.2. Since $J$ is an $m$-PD ideal, we have $a^{p^{m+1}}=p ! a^{\left\{p^{m+1}\right\}}=0$ in characteristic $p>0$ for any $a \in J$. Therefore $U \rightarrow T$ is a homeomorphism. Since $a^{p^{m+1}}=0$ for any $a \in J$, the morphism $\mathcal{O}_{S} \otimes_{\mathcal{O}_{S}} \mathcal{O}_{T} \rightarrow \mathcal{O}_{T} ; a \otimes b \mapsto a \cdot b^{p^{m+1}}$ induces a natural morphism $\mathcal{O}_{U^{\prime \prime}} \rightarrow \mathcal{O}_{T}$. Since $U^{\prime \prime} \rightarrow T^{\prime \prime}$ is strict, we also have a natural morphism $\mathcal{M}_{U^{\prime \prime}} \cong \mathcal{M}_{T^{\prime \prime}} \rightarrow \mathcal{M}_{T}$. Therefore the morphism $T \rightarrow T^{\prime \prime}$ factors through $U^{\prime \prime}$. We thus obtain solid arrows in the diagram

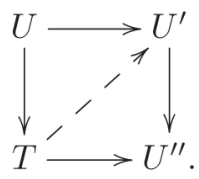

Then, since the morphism $U^{\prime} \rightarrow U^{\prime \prime}$ is log étale and $U \rightarrow T$ is an exact nilimmersion, there exists a unique morphism $T \rightarrow U^{\prime}$ (the dotted arrow) making the above diagram commute.

Definition 5.12. Let $(U, T, J, \delta)$ be an object in $\operatorname{CRIS}_{\mathrm{Int}}^{(m)}(X / S)$. We define the morphism $T \rightarrow X^{\prime}$ by the composition of the morphism in Lemma 5.11 and $U^{\prime} \rightarrow X^{\prime}$, and denote it by $f_{T / S}$.

If $g: T_{1} \rightarrow T_{2}$ be a morphism of $\operatorname{CRIS}_{\text {Int }}^{(m)}(X / S)$, then $f_{T_{2} / S} \circ g=f_{T_{1} / S}$. Hence, if $E^{\prime}$ is an $\mathcal{O}_{X^{\prime}}$-module, there exists a natural isomorphism

$$
\theta_{g}: g^{*} f_{T_{2} / S}^{*} E^{\prime \cong} f_{T_{1} / S}^{*} E^{\prime}
$$

Thus we have the following lemma.

LEMma 5.13. Let $E^{\prime}$ be an $\mathcal{O}_{X^{\prime}}$-module. The collection $\left\{f_{T / S}^{*} E^{\prime}, \theta_{g}\right\}$ defines a log $m$-crystal of $\mathcal{O}_{X / S}^{(m)}$-modules on $\mathrm{CRIS}_{\mathrm{Int}}^{(m)}(X / S)$. We denote it by $F_{X / S}^{*} E^{\prime}$, by abuse of notation.

Definition 5.14. Let $\tilde{T}$ be an object of $\operatorname{CRIS}_{\text {Int }}^{(m)}(X / \tilde{S})$ which is flat over $\tilde{S}$, and $T$ the closed subscheme defined by $p$. A lifting of $f_{T / S}$ to $\tilde{T}$ is a lifting $\tilde{F}: \tilde{T} \rightarrow \tilde{X}^{\prime}$ over $\tilde{S}$ modulo $p^{2} \cdot \mathcal{L}_{\mathcal{X} / \mathcal{S}}^{(m)}(\tilde{T})$ denotes the set of all such liftings, and $\mathcal{L}_{\mathcal{X} / \mathcal{S}, \tilde{T}}^{(m)}$ denotes the étale sheaf of sets on $\tilde{T}$ of local liftings of $f_{T / S}$. For a morphism $\tilde{g}: \tilde{T}_{1} \rightarrow \tilde{T}_{2}$ of $\mathrm{CRIS}_{\text {Int }}^{(m)}(X / S)$, we define the map $\mathcal{L}_{\mathcal{X} / \mathcal{S}}^{(m)}(\tilde{g}): \mathcal{L}_{\mathcal{X} / \mathcal{S}}\left(\tilde{T}_{2}\right) \rightarrow \mathcal{L}_{\mathcal{X} / \mathcal{S}}\left(\tilde{T}_{1}\right)$ by $\tilde{F} \mapsto \tilde{F} \circ \tilde{g}$. 
Let $\operatorname{CRIS}_{\text {Int }, f}^{(m)}(X / \tilde{S})$ denote the full subsite of $\operatorname{CRIS}_{\text {Int }}^{(m)}(X / \tilde{S})$ consisting of those objects which are flat over $\tilde{S}$. The family $\left\{\mathcal{L}_{\mathcal{X} / \mathcal{S}, \tilde{T}}^{(m)} \mid \tilde{T} \in \operatorname{CRIS}_{\operatorname{Int}, f}^{(m)}(X / \tilde{S})\right\}$ together with the family of transition maps $\mathcal{L}_{\mathcal{X} / \mathcal{S}}^{(m)}(\tilde{g})$ defines a sheaf of sets on $\operatorname{CRIS}_{\operatorname{Int}, f}^{(m)}(\tilde{X} / S)$.

Lemma 5.15. Let $(\tilde{U}, \tilde{T}, \tilde{J}, \tilde{\delta})$ be an object of $\mathrm{CRIS}_{\operatorname{Int}, f}^{(m)}(X / \tilde{S})$. Let $T$ denote the closed subscheme of $\tilde{T}$ defined by $p$. Then the sheaf $\mathcal{L}_{\mathcal{X} / \mathcal{S}, \tilde{T}}^{(m)}$ forms a torsor over $\mathcal{H o m}_{\mathcal{O}_{T}}\left(f_{T / S}^{*} \Omega_{X^{\prime} / S}^{1}, \mathcal{O}_{T}\right) \cong f_{T / S}^{*} \mathcal{T}_{X^{\prime} / S}$.

Proof. Let us consider the following diagram

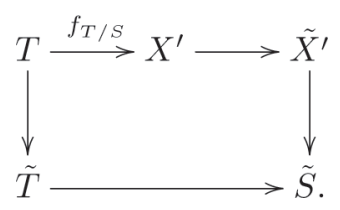

Since $\tilde{X}^{\prime} \rightarrow \tilde{S}$ is $\log$ smooth, a lifting of $f_{T / S}$ to $\tilde{T}$ exist locally on $T$, so $\mathcal{L}_{\mathcal{X} / \mathcal{S}, \tilde{T}}$ has nonempty stalks. If we define, for $g_{1}, g_{2} \in \mathcal{L}_{\mathcal{X} / \mathcal{S}, \tilde{T}}$, the subtraction $g_{1}-g_{2} \in$ $\mathcal{H o m}_{\mathcal{O}_{T}}\left(f_{T / S}^{*} \Omega_{X^{\prime} / S}^{1}, \mathcal{O}_{T}\right)$ by

$$
\begin{aligned}
& f_{T / S}^{*}(d x) \mapsto a \text { where } p \tilde{a}=g_{2}^{*}(\tilde{x})-g_{1}^{*}(\tilde{x}) \\
& f_{T / S}^{*}(d \log m) \mapsto b \text { where } g_{1}^{*}(\tilde{m})(1+p \tilde{b})=g_{2}^{*}(\tilde{m}),
\end{aligned}
$$

where $\tilde{a} \in \mathcal{O}_{\tilde{T}}$ (resp. $\tilde{a} \in \mathcal{O}_{\tilde{T}}$ ) is a lift of $a \in \mathcal{O}_{T}$ (resp. $b \in \mathcal{O}_{T}$ ) and $g_{i}^{*}$ is the underlying morphism of the structure sheaf or the log structure, then we can check that $\mathcal{L}_{\mathcal{X} / \mathcal{S}, \tilde{T}}$ is a torsor over $f_{T / S}^{*} \mathcal{T}_{X^{\prime} / S}$ by this subtraction.

Therefore the family $\left\{\mathcal{L}_{\mathcal{X} / \mathcal{S}, \tilde{T}}^{(m)}\right\}$ defines a $\log m$-crystal of torsors over $F_{X / S}^{*} \mathcal{T}_{X^{\prime} / S}$. The following lemma shows that this crystal of torsors on $\operatorname{CRIS}_{\operatorname{Int}, f}^{(m)}(X / \tilde{S})$ defines a $\log m$ crystal of torsors on $\mathrm{CRIS}_{\mathrm{Int}}^{(m)}(X / S)$.

Lemma 5.16. (1) The natural inclusion of sites $\operatorname{CRIS}_{\text {Int, } f}^{(m)}(X / \tilde{S}) \rightarrow \operatorname{CRIS}_{\text {Int }}^{(m)}(X / \tilde{S})$ induces an equivalence of categories between the respective categories of log m-crystals of $\mathcal{O}_{X / \tilde{S}}^{(m)}$-modules.

(2) The natural functor from the category of p-torsion log m-crystals of $\mathcal{O}_{X / \tilde{S}^{-}}^{(m)}$ modules on $\operatorname{CRIS}_{\text {Int }}^{(m)}(X / \tilde{S})$ to the category of log m-crystals of $\mathcal{O}_{X / S}^{(m)}$-modules on $\operatorname{CRIS}_{\text {Int }}^{(m)}(X / S)$ is an equivalence of categories.

(3) The natural functor from the category of p-torsion log m-crystals of torsors over $F_{X / S}^{*} \mathcal{T}_{X^{\prime} / S}$ on $\operatorname{CRIS}_{\text {Int }}^{(m)}(X / \tilde{S})$ to the category of log m-crystals of torsors over $F_{X / S}^{*} \mathcal{T}_{X^{\prime} / S}$ on $\mathrm{CRIS}_{\mathrm{Int}}^{(m)}(X / S)$ is an equivalence of categories. Here we regard $F_{X / S}^{*} \mathcal{T}_{X^{\prime} / S}$ as a log m-crystal of $\mathcal{O}_{X / \tilde{S}}^{(m)}$-modules on $\mathrm{CRIS}_{\mathrm{Int}}^{(m)}(X / \tilde{S})$ by (2). 
Proof. First, we prove (1). Since the question is étale local on $X$, we may assume that there exists a lifting $\tilde{X} / \tilde{S}$. Then, by Proposition 5.6, both categories can be identified with the category of $\mathcal{O}_{\tilde{X}}$-modules equipped with log hyper $m$-PD stratifications. Second, we prove (2). Since the question is étale local on $X$, we may assume that there exists a lifting $\tilde{X} / \tilde{S}$. Let $i: X \hookrightarrow \tilde{X}$ be the exact closed immersion. Then, by Proposition 5.6, the former category (resp. the latter category) can be identified with the category of $p$-torsion $\mathcal{O}_{\tilde{X}}$-modules (resp. $\mathcal{O}_{X}$-modules) equipped with $\log$ hyper $m$-PD stratifications. Let $(\mathcal{E}, \varepsilon)$ be an $\mathcal{O}_{X}$-module with a log hyper $m$-PD stratification. Then $i_{*} \mathcal{E}$ naturally forms a $p$-torsion $\mathcal{O}_{\tilde{X}}$-module. We can also endow $i_{*} \mathcal{E}$ with a $\log$ hyper $m$-PD stratification by using $\varepsilon$ in a natural way. This gives a quasi-inverse. Finally, we can prove (3) in the same way as (2).

Now we construct the $\mathcal{O}_{X}$-module $\mathcal{K}_{\mathcal{X} / \mathcal{S}}^{(m)}$ with natural actions of $\hat{\Gamma} .\left(F_{X / S}^{*} \mathcal{T}_{X^{\prime} / S}\right)$ and $\mathcal{D}_{X / S}^{(m)}$. Let $(\tilde{U}, \tilde{T}, \tilde{J}, \tilde{\delta})$ be an object of $\operatorname{CRIS}_{\text {Int }, f}^{(m)}(X / \tilde{S})$. Let $T$ be a closed subscheme of $\tilde{T}$ defined by $p$. In Lemma 5.15, we saw that $\mathcal{L}_{\mathcal{X} / \mathcal{S}, \tilde{T}}^{(m)}$ forms a torsor over $f_{T / S}^{*} \mathcal{T}_{X^{\prime} / S}$. For a local section $a \in \mathcal{L}_{\mathcal{X} / \mathcal{S}, \tilde{T}}^{(m)}$ and $\varphi \in \mathcal{H o m}\left(\mathcal{L}_{\mathcal{X} / \mathcal{S}, \tilde{T}}^{(m)}, \mathcal{O}_{T}\right)$, we define the map $\varphi_{a}: f_{T / S}^{*} \mathcal{T}_{X^{\prime} / S} \rightarrow \mathcal{O}_{T}$ by $D \mapsto \varphi(a+D)-\varphi(a)$. Let $\mathcal{E}_{\mathcal{X} / \mathcal{S}, \tilde{T}}^{(m)}$ denote the subsheaf of $\operatorname{Hom}\left(\mathcal{L}_{\mathcal{X} / \mathcal{S}, \tilde{T}}^{(m)}, \mathcal{O}_{T}\right)$ consisting of morphisms $\varphi: \mathcal{L}_{\mathcal{X} / \mathcal{S}, \tilde{T}}^{(m)} \rightarrow \mathcal{O}_{T}$ such that, for any local section $a$ of $\mathcal{L}_{\mathcal{X} / \mathcal{S}}^{(m)}$, the map $\varphi_{a}$ is $\mathcal{O}_{T}$-linear. Note that, for $\varphi \in \mathcal{E}_{\mathcal{X} / \mathcal{S}, \tilde{T}}^{(m)}$, the map $\varphi_{a}$ is independent of the choice of $a$. We put $\omega_{\varphi}:=\varphi_{a}$. Then, we have a diagram

$$
(\sharp)_{\tilde{T}} \quad 0 \longrightarrow \mathcal{O}_{T} \longrightarrow \mathcal{E}_{\mathcal{X} / \mathcal{S}, \tilde{T}}^{(m)} \underset{\varphi \mapsto \omega_{\varphi}}{\longrightarrow} f_{T / S}^{*} \Omega_{X^{\prime} / S}^{1} \longrightarrow 0,
$$

where the map $\mathcal{O}_{T} \rightarrow \mathcal{E}_{\mathcal{X} / \mathcal{S}, \tilde{T}}^{(m)}$ sends $b \in \mathcal{O}_{T}$ to the constant function. This is a locally split exact sequence. In fact, given a local section $a \in \mathcal{L}_{\mathcal{X} / \mathcal{S}, \tilde{T}}^{(m)}$, we define the map $\sigma_{a}: f_{T / S}^{*} \Omega_{X^{\prime} / S}^{1} \rightarrow \mathcal{E}_{\mathcal{X} / \mathcal{S}, \tilde{T}}^{(m)}$ by $\omega \mapsto[b \mapsto\langle\omega, b-a\rangle]$. Then this map gives a section of $\mathcal{E}_{\mathcal{X} / \mathcal{S}, \tilde{T}}^{(m)} \rightarrow f_{T / S}^{*} \Omega_{X^{\prime} / \mathcal{S}}^{1}$. The injection $\mathcal{O}_{T} \hookrightarrow \mathcal{E}_{\mathcal{X} / \mathcal{S}, \tilde{T}}^{(m)}$ induces an injection $S^{n}\left(\mathcal{E}_{\mathcal{X} / \mathcal{S}, \tilde{T}}^{(m)}\right) \hookrightarrow$ $S^{n+1}\left(\mathcal{E}_{\mathcal{X} / \mathcal{S}, \tilde{T}}^{(m)}\right)$ for each natural number $n$. We define the $\mathcal{O}_{T}$-algebra $\mathcal{K}_{\mathcal{X} / \mathcal{S}, \tilde{T}}^{(m)}$ by the inductive limit $S \cdot\left(\mathcal{E}_{\mathcal{X} / \mathcal{S}, \tilde{T}}^{(m)}\right):=\lim _{\rightarrow} S^{n}\left(\mathcal{E}_{\mathcal{X} / \mathcal{S}, \tilde{T}}^{(m)}\right)$.

Next, let us define an action of $f_{T / S}^{*} \mathcal{T}_{X^{\prime} / S}$ on $\mathcal{K}_{\mathcal{X} / \mathcal{S}, \tilde{T}}^{(m)}$. When the exact sequence $(\sharp)_{\tilde{T}}$ splits, we have $\mathcal{E}_{\mathcal{X} / \mathcal{S}, \tilde{T}}^{(m)} \cong \mathcal{O}_{T} \oplus f_{T / S}^{*} \Omega_{X^{\prime} / S}^{1}$. This isomorphism induces an isomorphism of $\mathcal{O}_{T^{-} \text {-algebras }} \mathcal{K}_{\mathcal{X} / \mathcal{S}, \tilde{T}}^{(m)} \cong S\left(f_{T / S}^{*} \Omega_{X^{\prime} / S}^{1}\right)$. Now we define an action of $D \in f_{T / S}^{*} \mathcal{T}_{X^{\prime} / S}$ on $\mathcal{K}_{\mathcal{X} / \mathcal{S}, \tilde{T}}^{(m)}$ by the composition

$$
\mathcal{K}_{\mathcal{X} / \mathcal{S}, \tilde{T}}^{(m)} \cong S\left(f_{T / S}^{*} \Omega_{X^{\prime} / S}^{1}\right) \stackrel{D}{\rightarrow} S \cdot\left(f_{T / S}^{*} \Omega_{X^{\prime} / S}^{1}\right) \cong \mathcal{K}_{\mathcal{X} / \mathcal{S}, \tilde{T}}^{(m)},
$$

where the map $D$ is defined as a derivation. Furthermore, this action induces an action of $\hat{\Gamma}$. $\left(f_{T^{\prime} / \mathcal{S}}^{*} \mathcal{T}_{X^{\prime} / S}\right)$ on $\mathcal{K}_{\mathcal{X} / \mathcal{S}, \tilde{T}}^{(m)}$. Since $D \in f_{T / S}^{*} \mathcal{T}_{X^{\prime} / S}$ acts on $\mathcal{K}_{\mathcal{X} / \mathcal{S}, \tilde{T}}^{(m)}$ as a derivation, the action of $D \in f_{T / S}^{*} \mathcal{T}_{X^{\prime} / S}$ on $\mathcal{O}_{T}$ is zero. Thus the action is independent of the 
choice of a splitting $\mathcal{E}_{\mathcal{X} / \mathcal{S}, \tilde{T}}^{(m)} \cong \mathcal{O}_{T} \oplus f_{T / S}^{*} \Omega_{X^{\prime} / S}^{1}$ of the exact sequence $(\sharp)_{\tilde{T}}$. Now the family $\left\{f_{T / S}^{*} \mathcal{T}_{X^{\prime} / S}\right\}$ (resp. $\left\{f_{T / S}^{*} \Omega_{X^{\prime} / S}^{1}\right\},\left\{\mathcal{E}_{\mathcal{X} / \mathcal{S}, \tilde{T}}^{(m)}\right\}$ and $\left\{\mathcal{K}_{\mathcal{X} / \mathcal{S}, \tilde{T}}^{(m)}\right\}$ ) defines a $p$-torsion $\log m$-crystal of $\mathcal{O}_{X / \tilde{S}}^{(m)}$-modules on $\mathrm{CRIS}_{\text {Int }, f}^{(m)}(X / \tilde{S})$. Hence, by Lemma 5.16, we obtain corresponding log $m$-crystals of $\mathcal{O}_{X / S}^{(m)}$-modules $F_{X / S}^{*} \mathcal{T}_{X^{\prime} / S}, F_{X / S}^{*} \Omega_{X^{\prime} / S}^{1}, \mathcal{E}_{\mathcal{X} / \mathcal{S}}^{(m)}$ and $\mathcal{K}_{\mathcal{X} / \mathcal{S}}^{(m)}$ respectively. Each of the log $m$-crystals defines an $\mathcal{O}_{X}$-module. We denote it by the same symbol. The family of exact sequences $\left\{(\sharp)_{\tilde{T}}\right\}$ induces an exact sequence of $\mathcal{O}_{X}$-modules

$$
(\sharp) \quad 0 \longrightarrow \mathcal{O}_{X} \longrightarrow \mathcal{E}_{\mathcal{X} / \mathcal{S}}^{(m)} \longrightarrow F_{X / S}^{*} \Omega_{X^{\prime} / S}^{1} \longrightarrow 0 .
$$

Let us assume that there exists a lifting $\tilde{X} \rightarrow \tilde{X}^{\prime}$ of $X \rightarrow X^{\prime}$ modulo $p^{2}$. Then the exact sequence $(\sharp)$ coincides with $(\sharp)_{\tilde{X}}$ and splits. The splitting defines an action of $\hat{\Gamma} .\left(F_{X / S}^{*} \mathcal{T}_{X^{\prime} / S}\right)$ on $\mathcal{K}_{\mathcal{X} / \mathcal{S}}^{(m)}$. Since this action is independent of the choice of a splitting, we have an action $(\mathrm{A})$ of $\hat{\Gamma} .\left(F_{X / S}^{*} \mathcal{T}_{X^{\prime} / S}\right)$ on $\mathcal{K}_{\mathcal{X} / \mathcal{S}}^{(m)}$ globally on $X$. On the other hand, the structure of $\log m$-crystal of $\mathcal{O}_{X / S}^{(m)}$-modules on $\mathcal{K}_{\mathcal{X} / \mathcal{S}}^{(m)}$ gives the action of $\mathcal{D}_{X / S}^{(m)}$ on $\mathcal{K}_{\mathcal{X} / \mathcal{S}}^{(m)}$. Thus we also have an action $(\mathrm{B})$ of $\mathcal{T}_{X^{\prime} / S}$ on $\mathcal{K}_{\mathcal{X} / \mathcal{S}}^{(m)}$ via the $p^{m+1}$-curvature map $\beta: \mathcal{T}_{X^{\prime} / S} \rightarrow \mathcal{D}_{X / S}^{(m)}$. Let us show the following lemma.

\section{Lemma 5.17. The two $\mathcal{T}_{X^{\prime} / S^{-}}$actions $(\mathrm{A})$ and $(\mathrm{B})$ on $\mathcal{K}_{\mathcal{X} / \mathcal{S}}^{(m)}$ are equal.}

Proof. Since the assertion is étale local, we may assume that there exists a lifting $\tilde{F}: \tilde{X} \rightarrow \tilde{X}^{\prime}$ of $X \rightarrow X^{\prime}$ modulo $p^{2}$ and a logarithmic system of coordinates $\left\{\tilde{m}_{i}\right\}_{i}$ of $\tilde{X} \rightarrow \tilde{S}$. Denote by $\left\{m_{i}\right\}_{i}$ the image of $\left\{\tilde{m}_{i}\right\}_{i}$ in $\mathcal{M}_{X}^{g p}$. Then $\left\{\pi^{*}\left(m_{i}\right)\right\}_{i}$ forms a a logarithmic system of coordinates of $X^{\prime} \rightarrow S$. Let $\left\{\xi_{i}^{\prime}\right\}_{i}$ denote the dual basis of $\mathcal{T}_{X^{\prime} / S}$ associated to $\left\{d \log \pi^{*}\left(m_{i}\right)\right\}_{i}$. We take a lift $\tilde{m}_{i}^{\prime} \in \mathcal{M}_{\tilde{X}^{\prime}}$ of $\pi^{*}\left(m_{i}\right)$. We denote by $\tilde{P}$ the $\log m$-PD envelope of the diagonal $\tilde{X} \rightarrow \tilde{X} \times_{\tilde{S}} \tilde{X}$ and by $P$ the log $m$-PD envelope of $X \rightarrow X \times_{S} X$. Let $\left\{\tilde{\eta}^{\{k\}}\right\}$ denote a basis of $\mathcal{O}_{\tilde{P}}$ induced by $\left\{\tilde{m}_{i}\right\}_{i}$. We denote the image of $\left\{\underline{\tilde{\eta}}^{\{\underline{k}\}}\right\}$ in $\mathcal{O}_{P}$ by $\left\{\underline{\eta}^{\{\underline{k}\}}\right\}$.

By the construction of $\mathcal{K}_{\mathcal{X} / \mathcal{S}}^{(m)}$ (and the actions of $\mathcal{T}_{X^{\prime} / S}$ on it), it suffices to show that the two actions (A) and (B) agree on $\mathcal{E}_{\mathcal{X} / \mathcal{S}}^{(m)}$. By the existence of a lifting $\tilde{F}: \tilde{X} \rightarrow \tilde{X}^{\prime}$, the exact sequence $(\sharp)$ splits via $\sigma_{\tilde{F}}: F_{X / S}^{*} \Omega_{X^{\prime} / S}^{1} \hookrightarrow \mathcal{E}_{\mathcal{X} / \mathcal{S}}^{(m)}$. Hence we have $\mathcal{E}_{\mathcal{X} / \mathcal{S}}^{(m)} \cong \mathcal{O}_{X} \oplus$ $F_{X / S}^{*} \Omega_{X^{\prime} / S}^{1}$. The two actions (A) and (B) are zero on $\mathcal{O}_{X}$. Thus, we need to calculate the action on $F_{X / S}^{*} \Omega_{X^{\prime} / S}^{1}$. The action (A) of $\xi_{i}^{\prime} \in \mathcal{T}_{X^{\prime} / S}$ is given by $F_{X / S}^{*} \Omega_{X^{\prime} / S}^{1} \rightarrow$ $\mathcal{O}_{X} ; d \log \pi^{*}\left(m_{j}\right) \mapsto \delta_{i j}$. On the other hand, the action (B) of $\xi_{i}^{\prime} \in \mathcal{T}_{X^{\prime} / S}$ is given by

$$
\begin{gathered}
F_{X / S}^{*} \Omega_{X^{\prime} / S}^{1} \stackrel{\sigma_{\tilde{F}}}{\longrightarrow} \mathcal{E}_{\mathcal{X} / \mathcal{S}}^{(m)} \stackrel{p_{1}^{*}}{\longrightarrow} \mathcal{O}_{\tilde{P}} \otimes \mathcal{E}_{\mathcal{X} / \mathcal{S}}^{(m)} \stackrel{\cong}{\longrightarrow} \mathcal{E}_{\mathcal{X} / \mathcal{S}}^{(m)} \otimes \mathcal{O}_{\tilde{P}} \\
=\bigoplus_{\underline{k}} \mathcal{E}_{\mathcal{X} / \mathcal{S}}^{(m)} \underline{\eta}^{\{\underline{k}\}} \rightarrow \mathcal{E}_{\mathcal{X} / \mathcal{S}}^{(m)} \eta_{i}^{\left\{p^{m+1}\right\}}=\mathcal{E}_{\mathcal{X} / \mathcal{S}}^{(m)},
\end{gathered}
$$

where the isomorphism $\mathcal{O}_{\tilde{P}} \otimes \mathcal{E}_{\mathcal{X} / \mathcal{S}}^{(m)} \cong \mathcal{E}_{\mathcal{X} / \mathcal{S}}^{(m)} \otimes \mathcal{O}_{\tilde{P}}$ is the HPD-stratification asso- 
ciated to the $\log m$-crystal structure on $\mathcal{E}_{\mathcal{X} / \mathcal{S}}^{(m)}$, and the last map is the natural projection. Let us calculate this action explicitly. First, as a map $\mathcal{L}_{\mathcal{X} / \mathcal{S}, \tilde{X}}^{(m)} \rightarrow \mathcal{O}_{X}$, $\sigma_{\tilde{F}}\left(d \log \pi^{*}\left(m_{i}\right)\right) \in \mathcal{E}_{\mathcal{X} / \mathcal{S}}^{(m)}$ sends $\tilde{F}^{\prime} \in \mathcal{L}_{\mathcal{X} / \mathcal{S}, \tilde{X}}^{(m)}$ to $a$, where $a$ is the section of $\mathcal{O}_{X}$ satisfying $\tilde{F}^{*}\left(\tilde{m}_{j}^{\prime}\right)(1+p \tilde{a})=\tilde{F}^{\prime}\left(\tilde{m}_{j}^{\prime}\right)$. (We denote by $\sim$ a lifting of a section.) Next, we take the pullback of $\sigma_{\tilde{F}}\left(d \log \pi^{*}\left(m_{i}\right)\right)$ by $p_{1}^{*}$ and obtain the map $\sigma_{p_{1} \circ \tilde{F}}\left(d \log \pi^{*}\left(m_{i}\right)\right)$ : $\mathcal{L}_{\mathcal{X} / \mathcal{S}, \tilde{P}}^{(m)} \rightarrow \mathcal{O}_{P}$. We consider the composition of maps

$$
\mathcal{L}_{\mathcal{X} / \mathcal{S}, \tilde{X}}^{(m)} \stackrel{-\circ p_{0}}{\longrightarrow} \mathcal{L}_{\mathcal{X} / \mathcal{S}, \tilde{P}}^{(m)} \stackrel{\sigma_{p_{1} \tilde{\tilde{F}}}\left(d \log \pi^{*}\left(m_{i}\right)\right)}{\longrightarrow} \mathcal{O}_{P} .
$$

Then, this map sends $\quad \tilde{F}^{\prime} \in \mathcal{L}_{\mathcal{X} / \mathcal{S}, \tilde{X}}^{(m)} \quad$ to $\quad b \in \mathcal{O}_{P}=\bigoplus \mathcal{O}_{X} \underline{\eta}^{\{\underline{k}\}} \quad$ satisfying $\left(\tilde{F} \circ p_{1}\right)^{*}\left(\tilde{m_{j}^{\prime}}\right)(1+p \tilde{b})=\left(\tilde{F}^{\prime} \circ p_{0}\right)^{*}\left(\tilde{m_{j}^{\prime}}\right)$. We have to show that the $\eta_{i}^{\left\{p^{m+1}\right\}}$-component of $b$ is $\delta_{i j}$. Let us take $c, d \in \mathcal{O}_{P}$ satisfying

$$
\begin{aligned}
& \left(\tilde{F} \circ p_{1}\right)^{*}\left(\tilde{m}_{j}^{\prime}\right)(1+p \tilde{c})=\left(\tilde{F} \circ p_{0}\right)^{*}\left(\tilde{m}_{j}^{\prime}\right), \\
& \left(\tilde{F} \circ p_{0}\right)^{*}\left(\tilde{m}_{j}^{\prime}\right)(1+p \tilde{d})=\left(\tilde{F}^{\prime} \circ p_{0}\right)^{*}\left(\tilde{m_{j}^{\prime}}\right) .
\end{aligned}
$$

Then, we have $b=c+d$. By the definition of $d$, we may assume that $\tilde{d}$ is of the form $\tilde{d}=p_{0}^{*}\left(\tilde{d}^{\prime}\right)$. Then $d$ is in $\mathcal{O}_{X} \cdot 1 \subset \mathcal{O}_{P}$, and we see that the $\eta_{i}^{\left\{p^{m+1}\right\}}$-component of $d$ is zero. On the other hand, From (15), we have

$$
p_{1}^{*}\left(\tilde{F}^{*}\left(\tilde{m}_{j}^{\prime}\right)\right)(1+p \tilde{c})=p_{0}^{*}\left(\tilde{F}^{*}\left(\tilde{m}_{j}^{\prime}\right)\right) .
$$

Since $\tilde{F}^{*}\left(\tilde{m}_{j}^{\prime}\right)$ is a lift of $F_{X / S}^{*} \pi^{*}\left(m_{j}\right)=m_{j}^{p^{m+1}}$, there exists $\tilde{e} \in \mathcal{O}_{\tilde{X}}$ satisfying

$$
\tilde{F}^{*}\left(\tilde{m}_{j}^{\prime}\right)=\tilde{m}_{j}^{p^{m+1}} \cdot(1+p \tilde{e}) .
$$

From (16) and (17), we have

$$
\left(1+p\left(p_{1}^{*}(\tilde{e})-p_{0}^{*}(\tilde{e})+\tilde{c}\right)\right)\left(1+\tilde{\eta}_{j}\right)^{p^{m+1}}=1 .
$$

Now, since $\left(1+\tilde{\eta}_{j}\right)^{p^{m+1}}=1+p\left(\right.$ the terms of $\tilde{\eta}_{j}$ of degree $\left.<p^{m+1}\right)+p ! \tilde{\eta}_{j}^{\left\{p^{m+1}\right\}}$, we see that

$$
\text { the } \eta_{i}^{\left\{p^{m+1}\right\}} \text {-component of }\left(p_{1}^{*}(e)-p_{0}^{*}(e)+c\right)=\delta_{i j} \text {. }
$$

Finally, since $p_{1}^{*}(e)-p_{0}^{*}(e)=\sum_{\underline{k}>0} \underline{\partial}_{\langle\underline{k}\rangle}(e) \underline{\eta}^{\{\underline{k}\}}$,

$$
\text { the } \eta_{j}^{\left\{p^{m+1}\right\}} \text {-component of }\left(p_{1}^{*}(e)-p_{0}^{*}(e)\right)=\underline{\partial}_{\left\langle p^{m+1} \underline{\underline{\varepsilon_{j}}}\right\rangle}(e)=0 \text {. }
$$

Hence the $\eta_{j}^{\left\{p^{m+1}\right\}}$-component of $c$ is $\delta_{i j}$. We finish the proof.

Now, we are ready to construct the log global Cartier transform of higher level. Let $\check{\mathcal{K}}_{\mathcal{X} / \mathcal{S}}^{(m)}$ be the dual of $\mathcal{K}_{\mathcal{X} / \mathcal{S}}^{(m)}$ as a left $\mathcal{D}_{X / S}^{(m)}$-module. We consider the tensor product $\check{\mathcal{K}}_{\mathcal{X} / \mathcal{S}}^{(m), \mathcal{A}}:=\mathcal{A}_{X}^{g p} \otimes_{\mathcal{O}_{X}} \check{\mathcal{K}}_{\mathcal{X} / \mathcal{S}}^{(m)}$ as a left $\mathcal{D}_{X / \mathcal{S}}^{(m)}$-module. Let us show that $\check{\mathcal{K}}_{\mathcal{X} / \mathcal{S}}^{(m), \mathcal{A}}$ is a splitting 
module for $\tilde{\mathcal{D}}_{X / \mathcal{S}}^{(m)}$ over $\mathcal{O}_{\mathcal{G}}^{\mathcal{B}}$. Since the action of $\mathcal{D}_{X / \mathcal{S}}^{(m)}$ on $\check{\mathcal{K}}_{\mathcal{X} / \mathcal{S}}^{(m), \mathcal{A}}$ is admissible by Remark 4.25, the action of $\mathcal{D}_{X / S}^{(m)}$ extends to an $\mathcal{J}$-indexed $\tilde{\mathcal{D}}_{X / S}^{(m)}$-module structure on $\check{\mathcal{K}}_{\mathcal{X} / \mathcal{S}}^{(m), \mathcal{A}}$. On the other hand, we also have the $\mathcal{O}_{\mathcal{G}}$-action on $\check{\mathcal{K}}_{\mathcal{X} / \mathcal{S}}^{(m)}$ induced from the action (A). By Lemma 5.17, these two actions extend to the structure of left $\mathcal{A}_{X}^{g p} \otimes_{\mathcal{O}_{X}} \mathcal{D}_{X / S}^{(m)} \otimes_{S \cdot \mathcal{T}^{\prime} / \mathcal{S}} \mathcal{O}_{\mathcal{G}} \cong \tilde{\mathcal{D}}_{X}^{(m), \gamma}$-modules on $\check{\mathcal{K}}_{\mathcal{X} / \mathcal{S}}^{(m), \mathcal{A}}$. Since we locally have an isomorphism

$$
\check{\mathcal{K}}_{\mathcal{X} / \mathcal{S}}^{(m)}:=\mathcal{H o m}_{\mathcal{O}_{X}}\left(\mathcal{K}_{\mathcal{X} / \mathcal{S}}^{(m)}, \mathcal{O}_{X}\right) \cong \hat{\Gamma} .\left(F_{X / S}^{*} \mathcal{T}_{X^{\prime} / S}\right) \cong F_{X / S}^{*} \mathcal{O}_{\mathcal{G}},
$$

$\check{\mathcal{K}}_{\mathcal{X} / \mathcal{S}}^{(m), \mathcal{A}}$ is a locally free $\mathcal{O}_{\mathcal{G}}^{\mathcal{A}}$-module of rank 1 . Moreover, by Proposition 4.13, $\mathcal{O}_{\mathcal{G}}^{\mathcal{A}} \cong \mathcal{O}_{\mathcal{G}}^{\mathcal{B}} \otimes_{\mathcal{B}_{X \mathcal{S}}^{(m+1)}} \mathcal{A}_{X}^{g p}$ is a locally free module of rank $p^{(m+1) r}$ over $\mathcal{O}_{\mathcal{G}}^{\mathcal{B}}$. Hence $\check{\mathcal{K}}_{\mathcal{X} / \mathcal{S}}^{(m), \mathcal{A}}$ is a splitting module for $\tilde{\mathcal{D}}_{X / S}^{(m)}$ over $\mathcal{O}_{\mathcal{G}}^{\mathcal{B}}$ by Proposition 2.9. We thus obtain the following isomorphism of $\mathcal{O}_{\mathcal{G}}^{\mathcal{B}}$-algebras:

$$
\tilde{\mathcal{D}}_{X / \mathcal{S}}^{(m)} \otimes_{\tilde{Z}} \mathcal{O}_{\mathcal{G}}^{\mathcal{B}} \cong \mathcal{E} n d_{\mathcal{O}_{\mathcal{G}}^{\mathcal{B}}}\left(\check{\mathcal{K}}_{\mathcal{X} / \mathcal{S}}^{(m), \mathcal{A}}\right) .
$$

REmark 5.18. In the case without log structure, Gros, Le Stum and Quirós obtained a similar isomorphism (18) in a different way. See Subsection 6.4 of [4].

Using Proposition 2.7, we obtain the following theorem, which is the central result of this paper.

TheoRem 5.19. Let $\mathcal{X} / \mathcal{S}=\left(X \rightarrow S, \tilde{X}^{\prime} \rightarrow \tilde{S}\right)$ be a log smooth integral morphism with a lifting $\tilde{X} \rightarrow \tilde{S}$ modulo $p^{2}$. Then the functor

$$
C_{\mathcal{X} / \mathcal{S}}: \operatorname{MIC}_{\mathrm{PD}}^{\mathcal{A}, \mathcal{J}}(X / S) \rightarrow \operatorname{HIG}_{\mathrm{PD}}^{\mathcal{B}, \mathcal{J}}\left(X^{\prime} / S\right), E \mapsto \mathcal{H o m}_{\tilde{\mathcal{D}}_{X}^{(m), y}}\left(\check{\mathcal{K}}_{\mathcal{X} / \mathcal{S}}^{(m), \mathcal{A}}, E\right)
$$

is an equivalence of categories. The quasi-inverse of $C_{\mathcal{X} / \mathcal{S}}$ is given by

$$
C_{\mathcal{X} / \mathcal{S}}^{-1}: \operatorname{HIG}_{\mathrm{PD}}^{\mathcal{B}, \mathcal{J}}\left(X^{\prime} / S\right) \rightarrow \operatorname{MIC}_{\mathrm{PD}}^{\mathcal{A}, \mathcal{J}}(X / S), E^{\prime} \mapsto \check{\mathcal{K}}_{\mathcal{X} / \mathcal{S}}^{(m), \mathcal{A}} \otimes_{\mathcal{O}_{\mathcal{G}}^{\mathcal{B}}} E^{\prime} .
$$

Let us give two corollaries. In the case $\mathcal{J}=\mathcal{I}_{X}^{g p}$ with the standard action, the category of $\mathcal{J}$-indexed $\mathcal{A}_{X}^{g p}$-modules is equivalent to the category of $\mathcal{O}_{X}$-modules with quasi-inverse $E \mapsto \mathcal{A}_{X}^{g p} \otimes_{\mathcal{O}_{X}} E$ (see p. 22 of [11]).

We put $\mathcal{D}_{X / S}^{(m), \gamma}:=\mathcal{D}_{X / S}^{(m)} \otimes_{3} \mathcal{O}_{\mathcal{G}}$, and denote by $\operatorname{MIC}_{\mathrm{PD}}(X / S)$ the category of $\mathcal{D}_{X / S}^{(m), \gamma}$-modules. We thus obtain the following corollary.

COROLlaRY. The functor

$$
C_{\mathcal{X} / \mathcal{S}}: \operatorname{MIC}_{\mathrm{PD}}(X / S) \rightarrow \operatorname{HIG}_{\mathrm{PD}}^{\mathcal{B} \mathcal{I}_{X}^{g p}}\left(X^{\prime} / S\right), E \mapsto \mathcal{H o m}_{\tilde{\mathcal{D}}_{X}^{(m), y}}\left(\check{\mathcal{K}}_{\mathcal{X} / \mathcal{S}}^{(m), \mathcal{A}}, \mathcal{A}_{X}^{g p} \otimes_{\mathcal{O}_{X}} E\right)
$$

is an equivalence of categories.

Next, we consider the case without $\log$ structure. Let $X \rightarrow S$ be a smooth morphism of schemes. We denote by $\operatorname{HIG}_{\mathrm{PD}}\left(X^{\prime} / S\right)$ the category of $\mathcal{O}_{\mathcal{G}}$-modules. 
Corollary. Let $\mathcal{X} / \mathcal{S}=\left(X \rightarrow S, \tilde{X}^{\prime} \rightarrow \tilde{S}\right)$ be a smooth morphism of schemes with a lifting $\tilde{X} \rightarrow \tilde{S}$ modulo $p^{2}$. Then the functor

$$
C_{\mathcal{X} / \mathcal{S}}: \operatorname{MIC}_{\mathrm{PD}}(X / S) \rightarrow \operatorname{HIG}_{\mathrm{PD}}\left(X^{\prime} / S\right), E \mapsto \mathcal{H} o m_{\mathcal{D}_{X / \mathcal{S}}^{(m), \mathcal{K}}}\left(\check{\mathcal{K}}_{\mathcal{X} / \mathcal{S}}^{(m)}, E\right)
$$

is an equivalence of categories.

\section{Compatibility}

In this section, we discuss the compatibility with the log global Cartier transform and the log Frobenius descent. Throughout this section, we fix a sheaf of $\mathcal{I}_{X / S}^{g p}$-sets $\mathcal{J}$. For a $\log$ smooth morphism $X \rightarrow S$ in characteristic $p$, we denote by $F$ (resp. $\left.F_{X / S}\right)$ the $m$-th relative Frobenius morphism $X \rightarrow X^{(m)}$ (resp. the $(m+1)$-st relative Frobenius morphism $\left.X \rightarrow X^{\prime}:=X^{(m+1)}\right)$ of $X \rightarrow S$ by abuse of notation.

\section{1 - Frobenius descent}

Let us briefly recall Montagnon's $\log$ Frobenius descent. Let $\mathcal{E}$ be a $\mathcal{J}$-indexed left $\tilde{\mathcal{D}}_{X / S}^{(m)}$-module. We consider the $\mathcal{J} \cong \mathcal{H} \mathcal{H o m} *(*, \mathcal{J})$-indexed sheaf defined by

$$
\mathbb{F}_{\mathcal{J}}(\mathcal{E}):=\mathcal{H o m}_{\mathcal{D}_{X / \mathcal{S}}^{(m)}}\left(F^{*} \mathcal{D}_{X^{(m)} / S}^{(0)}, \mathcal{E}\right)
$$

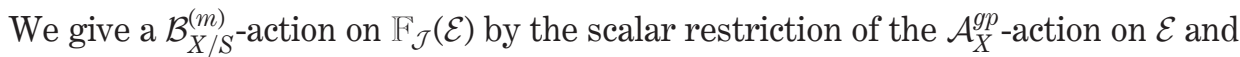
give a left $\mathcal{D}_{X^{(m)} / S}^{(0)}$-action on $\mathbb{F}_{\mathcal{J}}(\mathcal{E})$ by the right multiplication of $\mathcal{D}_{X^{(m)} / S}^{(0)}$ on $F^{*} \mathcal{D}_{X^{(m)} / S}^{(0)}$. Then one can see that these two actions naturally extend to a $\mathcal{J}$-indexed left $\tilde{\mathcal{D}}_{X^{(m)} / S^{-}}^{(0)}$ module structure on $\mathbb{F}_{\mathcal{J}}(\mathcal{E})$ (for the proof, see Subsection 4.2.1 of [9]). We thus obtain a functor

$$
\left(\mathcal{J} \text {-indexed left } \tilde{\mathcal{D}}_{X / S}^{(m)} \text {-modules }\right) \stackrel{\mathrm{F}_{\mathcal{J}}}{\longrightarrow}\left(\mathcal{J} \text {-indexed left } \tilde{\mathcal{D}}_{X^{(m)} / S}^{(0)} \text {-modules }\right) .
$$

REMARK 6.1. As is the case with the definition of $\mathcal{B}_{X / S}^{(m)}$, Montagnon defines the Frobenius descent functor by $\mathbb{F}(\mathcal{E}):=\mathcal{H o m}_{\mathcal{D}_{X / \mathcal{S}, \mathcal{I}}^{(m)}}\left(F^{*} \mathcal{D}_{X^{(m)} / S, \mathcal{I}}^{(0)}, \mathcal{E}\right)$. It seems to us that a natural definition of $\mathbb{F}(\mathcal{E})$ is $\mathcal{H}_{0 m_{\mathcal{D}_{X / S}^{(m)}}}\left(F^{*} \mathcal{D}_{X^{(m)} / S}^{(0)}, \mathcal{E}\right)$ because of the same reason as in Remark 4.3.

Next, let us construct a quasi inverse of $\mathbb{F}_{\mathcal{J}}$. Here we give some supplementary argument which was missing in [9]. Let $\mathcal{F}$ be a $\mathcal{J}$-indexed left $\tilde{\mathcal{D}}_{X^{(m)} / S^{(0)}}$-module. We consider $\mathcal{J} \stackrel{\cong}{\rightrightarrows} \mathcal{I}_{X}^{g p} \otimes_{\mathcal{I}_{X}^{g p}} \mathcal{J}$-indexed sheaf defined by

$$
\mathrm{G}_{\mathcal{J}}(\mathcal{F}):=\mathcal{A}_{X}^{g p} \otimes_{F^{*} \mathcal{B}_{X / S}^{(m)}} F^{*} \mathcal{F} .
$$


Here $F^{*} \mathcal{B}_{X / S}^{(m)}$ is the $\mathcal{I}_{X}^{g p \cong} \cong \otimes_{*} \mathcal{I}_{X}^{g p}$-indexed $\mathcal{O}_{X}$-algebra $\mathcal{O}_{X} \otimes_{F^{-1}} \mathcal{O}_{X^{(m)}} F^{-1} \mathcal{B}_{X / S}^{(m)}$ and $F^{*} \mathcal{F}$ is defined in a similar manner. $\mathrm{G}_{\mathcal{J}}(\mathcal{F})$ has an $\mathcal{A}_{X}^{g p}$-action induced from the first component. Let us endow $\mathrm{G}_{\mathcal{J}}(\mathcal{F})$ with a left $\mathcal{D}_{X / S^{-}}^{(m)}$-action in the following way. Let $\left\{\varepsilon_{\mathcal{A}, n}\right\}$ be the $\log m$-stratification of $\mathcal{A}_{X}^{g p}$ associated to its $\mathcal{D}_{X / S^{m}}^{(m)}$-action. Let $\left\{\varepsilon_{\mathcal{F}, n}\right\}$

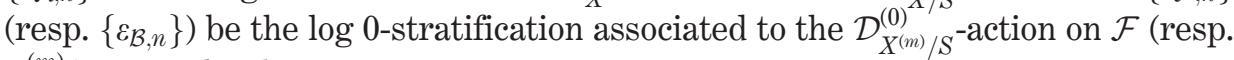
$\left.\mathcal{B}_{X / S}^{(m)}\right)$. Let $\Psi$ be the composition

$$
p_{1}^{n *} \mathcal{A}_{X}^{g p} \times p_{1}^{n *} F^{*} \mathcal{F} \stackrel{\varepsilon_{\mathcal{A}, n} \times \Phi^{*} \varepsilon_{\mathcal{F}, n}}{\longrightarrow} p_{0}^{n *} \mathcal{A}_{X}^{g p} \times p_{0}^{n *} F^{*} \mathcal{F} \rightarrow p_{0}^{n *} \mathcal{A}_{X}^{g p} \otimes_{p_{0}^{n *} F^{*} \mathcal{B}_{X / S}^{(m)}} p_{0}^{n *} F^{*} \mathcal{F},
$$

where $p_{0}^{n}$ and $p_{1}^{n}$ denote the first and second projection $P_{X / S}^{n} \rightarrow X, \Phi$ is defined in the beginning of Subsection 4.1.2 and the second map is the natural projection.

LeMma 6.2. $\Psi$ is a biadditive $p_{1}^{n *} F^{*} \mathcal{B}_{X / S^{(m)}}$-balanced map.

Proof. We prove $\Psi(a b, f)=\Psi(a, b f)$ for any local section $a \in p_{1}^{n *} \mathcal{A}_{X}^{g p}$, $b \in p_{1}^{n *} F^{*} \mathcal{B}_{X / \mathcal{S}}^{(m)}$ and $f \in p_{1}^{n *} F^{*} \mathcal{F}$. Since $\varepsilon_{\mathcal{A}, n}$ is a morphism of $\mathcal{I}_{X}^{g p}$-indexed $\mathcal{P}_{X / S,(m)}^{n}$ algebras, $\Psi(a b, f)$ is equal to $\varepsilon_{\mathcal{A}, n}(a) \varepsilon_{\mathcal{A}, n}(b) \otimes \Phi^{*} \varepsilon_{\mathcal{F}, n}(f)$. On the other hand, since $\mathcal{D}_{X^{(m)} / S}^{(0)}$-module structure on $\mathcal{F}$ is admissible (see Remark 4.25), $\Psi(a, b f)$ is equal to $\varepsilon_{\mathcal{A}, n}(a) \otimes \Phi^{*} \varepsilon_{\mathcal{B}, n}(b) \Phi^{*} \varepsilon_{\mathcal{F}, n}(f)$. So it suffices to show that $\varepsilon_{\mathcal{A}, n}(b)=\Phi^{*} \varepsilon_{\mathcal{B}, n}(b)$. This claim follows from Lemma 6.3 below.

LEMMA 6.3. The natural homomorphism $F^{*} \mathcal{B}_{X / S}^{(m)} \rightarrow \mathcal{A}_{X}^{g p}$ of $\mathcal{I}_{X}^{g p}$-indexed $\mathcal{O}_{X^{-}}$ algebras is a morphism of $\mathcal{I}_{X}^{g p}$-indexed $\mathcal{D}_{X / S}^{(m)}$-modules.

Proof. We may work étale locally on $X$. Let $\left\{\underline{\partial}_{\langle\underline{\hat{k}}\rangle}\right\}$ and $\left\{\underline{\partial}_{\langle\underline{x}\rangle}^{\prime}\right\}$ be as in Remark 4.6. Let $\psi$ denote the natural homomorphism of $\mathcal{I}_{X}^{g p}$-indexed $\mathcal{O}_{X}$-algebras defined by

$$
F^{*} \mathcal{B}_{X / S}^{(m)}=\mathcal{O}_{X} \otimes_{\mathcal{O}_{X^{(m)}}} \mathcal{H o m}_{\mathcal{D}_{X / S}^{(m)}}\left(F^{*} \mathcal{D}_{X^{(m)} / S}^{(0)}, \mathcal{A}_{X}^{g p}\right) \rightarrow \mathcal{A}_{X}^{g p}, \quad a \otimes f \mapsto a f(1 \otimes 1) .
$$

We shall show the equality $\psi\left(\underline{\partial}_{\langle k\rangle} \cdot(a \otimes f)\right)=\underline{\partial}_{\langle k\rangle} \cdot \psi(a \otimes f)$. By the formulas in Remark 4.6, the left hand side is calculated by

$$
\begin{aligned}
\psi\left(\underline{\partial}_{\langle\underline{k}\rangle} \cdot(a \otimes f)\right) & =\psi\left(\sum_{\underline{i} \leq \underline{k}}\left\{\begin{array}{c}
\underline{k} \\
\underline{i}
\end{array}\right\} \underline{\partial}_{\underline{\underline{k}}-\underline{i}\rangle}(a) \underline{\partial}_{\langle\underline{i}\rangle} \cdot(1 \otimes f)\right) \\
& =\psi\left(\sum_{p^{m} \underline{j} \underline{j} \underline{\underline{k}}}\left\{\begin{array}{c}
\underline{k} \\
p^{m} j
\end{array}\right\} \underline{\partial}_{\left\langle\underline{k}-p^{m} \underline{j}\right\rangle}(a) \otimes \underline{\partial}_{\langle\underline{j}\rangle}^{\prime} \cdot f\right) \\
& =\sum_{p^{m} \underline{j} \underline{j} \underline{k}}\left\{\begin{array}{c}
\underline{k} \\
p^{m} j
\end{array}\right\} \underline{\partial}_{\left\langle\underline{k}-p^{m} \underline{j}\right\rangle}(a) f\left(1 \otimes \underline{\partial}_{\langle\underline{j}\rangle}^{\prime}\right) .
\end{aligned}
$$


Similarly, by the formulas in Remark 4.6, the right hand side is calculated by

$$
\begin{aligned}
& \underline{\partial}_{\langle\underline{k}\rangle} \cdot a f(1 \otimes 1)=\sum_{\underline{i} \leq \underline{k}}\left\{\begin{array}{l}
\underline{k} \\
\underline{i}
\end{array}\right\} \underline{\partial}_{\langle\underline{k}-\underline{i}\rangle}(a) \underline{\partial}_{\langle\underline{i}\rangle} \cdot f(1 \otimes 1) \\
& =\sum_{\underline{i} \leq \underline{k}}\left\{\begin{array}{l}
\underline{k} \\
\underline{i}
\end{array}\right\} \underline{\partial}_{\langle\underline{k}-\underline{i}\rangle}(a) f\left(\underline{\partial}_{\langle\underline{i}\rangle} \cdot(1 \otimes 1)\right) \\
& =\sum_{p^{m} j \underline{\underline{k}} \underline{\underline{k}}}\left\{\begin{array}{c}
\underline{k} \\
p^{m} \underline{j}
\end{array}\right\} \underline{\partial}_{\left\langle\underline{k}-p^{m} \underline{j} \underline{\underline{j}}\right.}(a) f\left(1 \otimes \underline{\partial}_{\underline{j}>\rangle}^{\prime}\right) .
\end{aligned}
$$

This finishes the proof.

Thanks to Lemma 6.2 and the universal mapping property of a tensor product, we have

$$
\varepsilon_{n}: p_{1}^{n *} \mathcal{A}_{X}^{g p} \otimes_{p_{1}^{n *} F^{*} \mathcal{B}_{X / S}^{(m)}} p_{1}^{n *} F^{*} \mathcal{F} \rightarrow p_{0}^{n *} \mathcal{A}_{X}^{g p} \otimes_{p_{0}^{n *} F^{*} \mathcal{B}_{X / S}^{(m)}} p_{0}^{n *} F^{*} \mathcal{F} .
$$

One can obtain the inverse of $\varepsilon_{n}$ in a similar way. So these maps $\left\{\varepsilon_{n}\right\}$ are isomorphisms of $\mathcal{J}$-indexed $\mathcal{P}_{X / S,(m)}^{n}$-algebras. Furthermore $\left\{\varepsilon_{n}\right\}$ satisfy the obvious cocycle conditions. Therefore $\left\{\varepsilon_{n}\right\}$ form a $\log m$-stratification on $\mathcal{A}_{X}^{g p} \otimes_{F^{*} \mathcal{B}_{X / S}^{(m)}} F^{*} \mathcal{F}$ and defines a $\mathcal{J}$-indexed $\mathcal{D}_{X / S}^{(m)}$-module structure.

Lemma 6.4. The $\mathcal{J}$-indexed $\mathcal{D}_{X / S^{-m}}^{(m)}$-module structure on $\mathcal{A}_{X}^{g p} \otimes_{F^{*} B_{X / S}^{(m)}} F^{*} \mathcal{F}$ is admissible.

Proof. It suffices to show that the following diagram is commutative:

$$
\begin{gathered}
p_{1}^{n *}\left(\mathcal{A}_{X}^{g p} \otimes_{\mathcal{O}_{X}} \mathcal{A}_{X}^{g p} \otimes_{F^{*} \mathcal{B}_{X / S}^{(m)}} F^{*} \mathcal{F}\right) \longrightarrow p_{0}^{n *}\left(\mathcal{A}_{X}^{g p} \otimes_{\mathcal{O}_{X}} \mathcal{A}_{X}^{g p} \otimes_{F^{*} \mathcal{B}_{X / S}^{(m)}} F^{*} \mathcal{F}\right) \\
\downarrow \\
p_{1}^{n *}\left(\mathcal{A}_{X}^{g p} \otimes_{F^{*} \mathcal{B}_{X / S}^{(m)}} F^{*} \mathcal{F}\right) \longrightarrow \varepsilon_{n} \\
\downarrow
\end{gathered}
$$

where the upper horizontal arrow is the the $\log m$-stratification $\varepsilon_{\mathcal{A}, n} \otimes \varepsilon_{n}$ and the vertical arrows are induced from the multiplication on $\mathcal{A}_{X}^{g p}$. This follows from the fact that the $\log m$-stratification $\varepsilon_{\mathcal{A}, n}$ is a morphism of $\mathcal{I}_{X}^{g p}$-indexed $\mathcal{P}_{X / S,(m)}^{n}$-algebras.

By Lemma 6.4 and Remark 4.24 , we have a $\mathcal{J}$-indexed $\tilde{\mathcal{D}}_{X / S}^{(m)}$-module structure on $\mathcal{A}_{X}^{g p} \otimes_{F^{*} \mathcal{B}_{X / S}^{(m)}} F^{*} \mathcal{F}$ and obtain a functor

$\left(\mathcal{J}\right.$-indexed left $\tilde{\mathcal{D}}_{X^{(m)} / S}^{(0)}$-modules $) \stackrel{\mathrm{G}_{\mathcal{J}}}{\longrightarrow}\left(\mathcal{J}\right.$-indexed left $\tilde{\mathcal{D}}_{X / S^{(m)}}^{(m)}$-modules $)$.

THEOREM 6.5. $\mathbb{F}_{\mathcal{J}}$ is an equivalence of categories with a quasi-inverse $\mathrm{G}_{\mathcal{J}}$.

Proof. See Théorème 4.2.1 of [9]. 
REMARK 6.6. In [9], Montagnon only treats the case of $\mathcal{J}=\mathcal{I}_{X}^{g p}$, but the same argument works for any sheaf of $\mathcal{I}_{X}^{g p}$-sets $\mathcal{J}$.

\section{2 - Main Theorem}

Let us start with stating the main theorem. Let $\mathcal{X} / \mathcal{S}=\left(X \rightarrow S, \tilde{X}^{\prime} \rightarrow \tilde{S}\right)$ be a log smooth integral morphism of fine $\log$ schemes with a lifting $\tilde{X}^{\prime} \rightarrow \tilde{S}$ of $X^{\prime} \rightarrow S$ modulo $p^{2}$. Note that $\tilde{X}^{\prime} \rightarrow \tilde{S}$ can likewise be considered as a lifting of $\left(X^{(m)}\right)^{(1)}=X^{(m+1)}=X^{\prime}$ modulo $p^{2}$, and we will also denote the data $\left(X^{(m)} \rightarrow S, \tilde{X}^{\prime} \rightarrow \tilde{S}\right)$ by $\mathcal{X} / \mathcal{S}$ by abuse of notation. As in Subsection 5.2-, $\mathcal{G}$ denotes the nilpotent divided power envelope of the zero section of the cotangent bundle of $X^{\prime} / S, \mathcal{O}_{\mathcal{G}}^{\mathcal{B}}$ denotes $\mathcal{B}_{X / S}^{(m+1)} \otimes_{\mathcal{O}_{X^{\prime}}} \mathcal{O}_{\mathcal{G}}$ and $\operatorname{HIG}_{\mathrm{PD}}^{\mathcal{B}, \mathcal{J}}\left(X^{\prime} / S\right)$ denotes the category of $\mathcal{J}$-indexed $\mathcal{O}_{\mathcal{G}}^{\mathcal{B}}$-modules. We put $\tilde{\mathcal{D}}_{X^{(m)} / S}^{(0), \gamma}=$ $\tilde{\mathcal{D}}_{X^{(m)} / S}^{(0)} \otimes_{\mathcal{Y}^{\prime}} \mathcal{O}_{\mathcal{G}}^{\mathcal{B}}$, and denote by $\operatorname{MIC}_{\mathrm{PD}}^{\mathcal{B}, \mathcal{J}}\left(X^{(m)} / S\right)$ the category of $\mathcal{J}$-indexed $\tilde{\mathcal{D}}_{X^{(m)}}^{(0), S^{-}}$ modules. By Theorem 4.20, Proposition 4.14 and Lemma 5.17, we see that $\check{\mathcal{K}}_{\mathcal{X} / \mathcal{S}}^{(0), \mathcal{B}}:=\mathcal{B}_{X / S}^{(m)} \otimes_{\mathcal{O}_{X^{(m) / S}}} \check{\mathcal{K}}_{\mathcal{X} / \mathcal{S}}^{(0)}$ is a splitting module for $\tilde{\mathcal{D}}_{X^{(m) / S}}^{(0)}$ over $\mathcal{O}_{\mathcal{G}}^{\mathcal{B}}$ and we obtain an equivalence of categories

$$
C^{\prime}: \operatorname{MIC}_{\mathrm{PD}}^{\mathcal{B}, \mathcal{J}}\left(X^{(m)} / S\right) \rightarrow \operatorname{HIG}_{\mathrm{PD}}^{\mathcal{B}, \mathcal{J}}\left(X^{\prime} / S\right), E \mapsto \mathcal{H o m}_{\tilde{\mathcal{D}}_{X^{(m)} / \mathcal{S}}^{(0),}}\left(\check{\mathcal{K}}_{\mathcal{X} / \mathcal{S}}^{(0), \mathcal{B}}, E\right),
$$

which is a variant of Theorem 5.19 and Theorem 4.2 in [11]. Note that the equivalence $C^{\prime}$ does not coincide with Theorem 4.2 in [11] (see Remark 4.10).

On the other hand, the Frobenius descent induces the following equivalence of categories.

LEMma 6.7. The Frobenius descent functor $\mathbb{F}_{\mathcal{J}}$ induces an equivalence of categories between $\mathrm{MIC}_{\mathrm{PD}}^{\mathcal{A}, \mathcal{J}}(X / S)$ and $\mathrm{MIC}_{\mathrm{PD}}^{\mathcal{B}, \mathcal{J}}\left(X^{(m)} / S\right)$.

Proof. Let $\mathcal{E}$ be an object in $\operatorname{MIC}_{\mathrm{PD}}^{\mathcal{A}, \mathcal{J}}(X / S)$. Then the $\hat{\Gamma} \cdot \mathcal{T}_{X^{\prime} / S^{-}}$action on $\mathbb{F}_{\mathcal{J}}(\mathcal{E})$ is naturally induced from the $\hat{\Gamma} \cdot \mathcal{T}_{X^{\prime} / S}$-action on $\mathcal{E}$. We shall show that the following diagram is commutative:

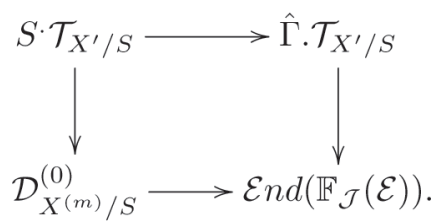

Here the left vertical arrow is the $p$-curvature map and the right vertical arrow (resp. the lower horizontal arrow) is induced from the $\hat{\Gamma} \cdot \mathcal{T}_{X^{\prime} / S^{-}}$action (resp. the

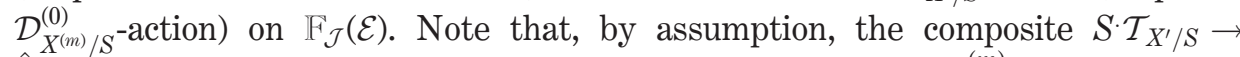
$\hat{\Gamma} \cdot \mathcal{T}_{X^{\prime} / S} \rightarrow \mathcal{E} n d\left(\mathbb{F}_{\mathcal{J}}(\mathcal{E})\right)$ is equal to the composite $S \cdot \mathcal{T}_{X^{\prime} / S} \rightarrow \mathcal{D}_{X / S}^{(m)} \rightarrow \mathcal{E} n d\left(\mathbb{F}_{\mathcal{J}}(\mathcal{E})\right)$, where the first arrow $S \cdot \mathcal{T}_{X^{\prime} / S} \rightarrow \mathcal{D}_{X / S}^{(m)}$ is the $p^{m+1}$-curvature map. 
We may work in a local situation. Take $g \in \mathbb{F}_{\mathcal{J}}(\mathcal{E})$. Then, by the formulas given in Remark 4.6, $S \cdot \mathcal{T}_{X^{\prime} / S}$-action on $\mathbb{F}_{\mathcal{J}}(\mathcal{E})$ defined by the composite $S \cdot \mathcal{T}_{X^{\prime} / S} \rightarrow$ $\mathcal{D}_{X^{(m)} / S}^{(0)} \rightarrow \mathcal{E} n d\left(\mathbb{F}_{\mathcal{J}}(\mathcal{E})\right)$ is calculated by

$$
\underline{\partial}_{\left\langle p \underline{\underline{\varepsilon}}_{i}\right\rangle(0)}^{\prime} \cdot g(1 \otimes 1)=g\left(1 \otimes \underline{\partial}_{\left\langle p \underline{\varepsilon}_{i}\right\rangle(0)}^{\prime}\right)=g\left(\underline{\partial}_{\left\langle p^{m+1} \underline{\underline{\varepsilon}}_{i}\right\rangle(m)} \cdot(1 \otimes 1)\right)=\underline{\partial}_{\left\langle p^{m+1} \underline{\underline{\varepsilon}}_{i}\right\rangle(m)} \cdot g(1 \otimes 1) .
$$

So this is the same as the action defined by the composite $S \cdot \mathcal{T}_{X^{\prime} / S} \rightarrow \mathcal{D}_{X / S}^{(m)} \rightarrow$ $\mathcal{E} n d\left(\mathbb{F}_{\mathcal{J}}(\mathcal{E})\right)$. Therefore these two $S \cdot \mathcal{T}_{X^{\prime} / S}$-actions are equal and $\mathbb{F}_{\mathcal{J}}(\mathcal{E})$ defines an object in $\operatorname{MIC}_{\mathrm{PD}}^{\mathcal{B}, \mathcal{J}}\left(X^{(m)} / S\right)$ if $\mathcal{E}$ is an object in $\operatorname{MIC}_{\mathrm{PD}}^{\mathcal{A}, \mathcal{J}}(X / S)$. One can check that a quasi-inverse $\mathrm{Gr} \mathcal{J}$ of $\mathbb{F}_{\mathcal{J}}$ induces the functor $\operatorname{MIC}_{\mathrm{PD}}^{\mathcal{B}, \mathcal{J}}\left(X^{(m)} / S\right) \rightarrow \mathrm{MIC}_{\mathrm{PD}}^{\mathcal{A}, \mathcal{J}}(X / S)$ in a similar manner.

Now, we are ready to state the main theorem in this section.

THEOREM 6.8. The following diagram of categories commutes:

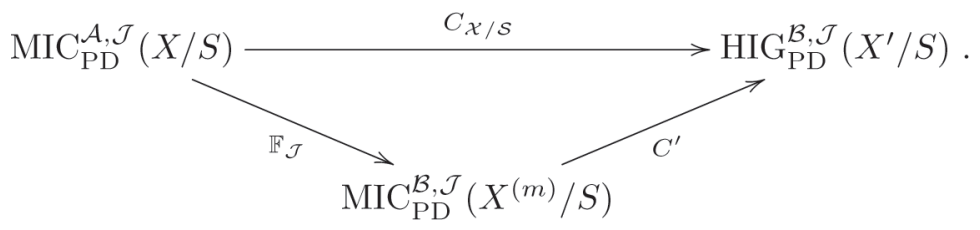

REMARK 6.9. Theorem 6.8 can be regarded as the log global version of the result stated in Subsection 6.6 of [4].

The key ingredient is the following.

THEOREM 6.10. The image of $\check{\mathcal{K}}_{\mathcal{X} / \mathcal{S}}^{(m), \mathcal{A}}$ under the Frobenius descent functor is naturally isomorphic to $\check{\mathcal{K}}_{\mathcal{X} / \mathcal{S}}^{(0), \mathcal{B}}$, that is,

$$
\mathbb{F}_{\mathcal{I}_{X}^{g p}}\left(\check{\mathcal{K}}_{\mathcal{X} / \mathcal{S}}^{(m), \mathcal{A}}\right)=\check{\mathcal{K}}_{\mathcal{X} / \mathcal{S}}^{(0), \mathcal{B}}
$$

We shall give a proof of Theorem 6.10 in the next subsection. Here, let us prove Theorem 6.8 from Theorem 6.10 . We need the following easy lemma.

Lemma 6.11. Let $\mathcal{A}$ be an $\mathcal{I}$-indexed $\mathcal{O}_{X}$-algebra. Let $\mathcal{E}$ be an $\mathcal{I}$-indexed left $\mathcal{A}$ module and $\mathcal{F}$ a $\mathcal{J}$-indexed left $\mathcal{A}$-module. Then, for each $j \in \mathcal{J}(X)=\operatorname{Hom}_{\mathcal{I}}(\mathcal{I}, \mathcal{J})$, $\mathcal{H o m}_{\mathcal{A}}(\mathcal{E}, \mathcal{F})(j)$ is isomorphic to $\operatorname{Hom}_{\mathcal{A}}(\mathcal{E}, \mathcal{F}(j))$.

Proof. There exists a natural morphism $\mathcal{H o m}_{\mathcal{A}}(\mathcal{E}, \mathcal{F})(j) \rightarrow \mathcal{H o m}{ }_{\mathcal{A}}(\mathcal{E}, \mathcal{F}(j))$. It suffices to show that this morphism is an isomorphism at every fiber. Let $i$ be a section of $\mathcal{I}$ defined on $U$. The right hand side is a sheaf defined by

$$
V \longmapsto \bigsqcup_{i^{\prime} \in \mathcal{I}(V)} \operatorname{Hom}_{\left.\mathcal{A}\right|_{V}}\left(\left.\mathcal{E}\right|_{V}, \mathcal{F}(j)\left(i^{\prime}\right)\right)=\bigsqcup_{i^{\prime} \in \mathcal{I}(V)} \operatorname{Hom}_{\left.\mathcal{A}\right|_{V}}\left(\left.\mathcal{E}\right|_{V}, \mathcal{F}\left(i^{\prime}+j\right)\right) .
$$


Therefore the fiber at $i$ is isomorphic to $\mathcal{H o m}_{\mathcal{A}}(\mathcal{E}, \mathcal{F}(i+j))$. On the other hand, the fiber of left hand side at $i$ is isomorphic to $\mathcal{H o m}_{\mathcal{A}}(\mathcal{E}, \mathcal{F})_{i+j} \cong \mathcal{H o m}_{\mathcal{A}}(\mathcal{E}, \mathcal{F}(i+j))$.

Now, we prove Theorem 6.8. Let $\mathcal{E}$ be an object in $\operatorname{MIC}_{\mathrm{PD}}^{\mathcal{A}, \mathcal{J}}(X / S)$ and consider the image

$$
C^{\prime}\left(\mathbb{F}_{\mathcal{J}}(\mathcal{E})\right)=\mathcal{H o m}_{\tilde{\mathcal{D}}_{X}^{(0), \vartheta}\left(m_{\mathcal{S}}\right.}\left(\check{\mathcal{K}}_{\mathcal{X} / \mathcal{S}}^{(0), \mathcal{B}}, \mathbb{F}_{\mathcal{J}}(\mathcal{E})\right)
$$

It is given as a presheaf on $X$ by

$$
U \longmapsto \bigsqcup_{j \in \mathcal{J}(U)} \operatorname{Hom}_{\left.\tilde{\mathcal{D}}_{\tilde{X}^{(m)}()_{\mathcal{S}}, \mathcal{Y}}\right|_{U}}\left(\left.\check{\mathcal{K}}_{\mathcal{X} / \mathcal{S}}^{(0), \mathcal{B}}\right|_{U}, \mathbb{F}_{\mathcal{J}}(\mathcal{E})(j)\right) .
$$

It suffices to show that $\operatorname{Hom}_{\tilde{\mathcal{D}}_{\left.X^{(0)}()_{S}\right)} \mid U}\left(\left.\check{\mathcal{K}}_{\mathcal{X} / \mathcal{S}}^{(0), \mathcal{B}}\right|_{U}, F_{\mathcal{J}}(\mathcal{E})(j)\right)$ is canonically identified with $\operatorname{Hom}_{\tilde{\mathcal{D}}_{X / \mathcal{S}}^{(m), y} \mid U}\left(\left.\check{\mathcal{K}}_{\mathcal{X} / \mathcal{S}}^{(m), \mathcal{A}}\right|_{U}, \mathcal{E}(j)\right)$ for every $U$ and $j \in \mathcal{J}(U)$. We may assume $U=X$. Then, by Theorem 6.10 and Lemma 6.11 , we have

$$
\operatorname{Hom}_{\tilde{\mathcal{D}}_{X^{(m) / \mathcal{S}}}^{(0), \gamma}}\left(\check{\mathcal{K}}_{\mathcal{X} / \mathcal{S}}^{(0), \mathcal{B}}, \mathbb{F}_{\mathcal{J}}(\mathcal{E})(j)\right)=\operatorname{Hom}_{\tilde{\mathcal{D}}_{X^{(m) / S}}^{(0), \gamma}}\left(\mathrm{F}_{\mathcal{I}_{X}^{g p}}\left(\check{\mathcal{K}}_{\mathcal{X} / \mathcal{S}}^{(m), \mathcal{A}}\right), \mathbb{F}_{\mathcal{I}_{X}^{g p}}(\mathcal{E}(j))\right) .
$$

The assertion follows from the fact that the Frobenius descent is an equivalence of categories.

\section{3 - Proof of Theorem 6.10}

In this subsection, we give a proof of Theorem 6.10. First, we introduce a description of $\mathrm{G}_{\mathcal{J}}$ in terms of crystals which is essentially due to Berthelot [1].

Let $\mathcal{E}$ be a log 0 -crystal on $\operatorname{CRIS}_{\mathrm{Int}}^{(0)}\left(X^{(m)} / \tilde{S}\right)$ and $(U, T, J, \delta)$ a log $m$-PD thickening in $\operatorname{CRIS}_{\text {Int }}^{(m)}(X / \tilde{S})$. We consider a closed subscheme $T_{0}$ of $T$ defined by $J+p \mathcal{O}_{T}$ and endow $T_{0}$ with the inverse image $\log$ structure from $T$. We have a log 0-PD thickening $T_{0} \hookrightarrow T$ and the following commutative diagram:

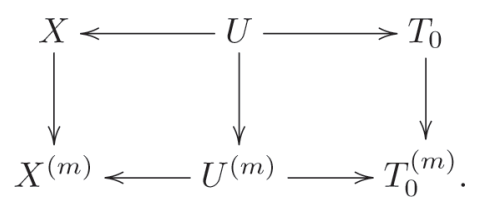

Here all vertical arrows are the $m$-th relative Frobenius morphisms.

Lemma 6.12. There exists a unique morphism $T_{0} \rightarrow U^{(m)}$ such that the following diagram commutes

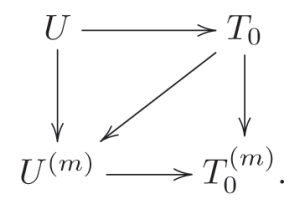


Proof. Note that all fine log schemes in the diagram are homeomorphic. Let $I$ denote the defining ideal of $U \hookrightarrow T$. Since $(U, T, J, \delta) \in \operatorname{CRIS}_{\text {Int }}^{(m)}(X / \tilde{S})$ (so $U$ and $T$ are integral over $\tilde{S}), \mathcal{O}_{U^{(m)}}=\mathcal{O}_{\tilde{S}} \otimes_{\mathcal{O}_{\tilde{S}}} \mathcal{O}_{T} / I \mathcal{O}_{\tilde{S}} \otimes_{\mathcal{O}_{\tilde{S}}} \mathcal{O}_{T}, \mathcal{O}_{T_{0}}=\mathcal{O}_{T} / J+p \mathcal{O}_{T}$. Consider the composition $\mathcal{O}_{T^{(m)}} \rightarrow \mathcal{O}_{T} \rightarrow \mathcal{O}_{T_{0}}$, where the first map is the $m$-th relative Frobenius and the second one is the natural projection. Then, since the image of this map is contained in $I^{\left(p^{m}\right)}+p I$, this one is zero on $I \mathcal{O}_{\tilde{S}} \otimes_{\mathcal{O}_{\tilde{S}}} \mathcal{O}_{T}$. Thus $\mathcal{O}_{T^{(m)}} \rightarrow \mathcal{O}_{T_{0}}$ uniquely factors as $\mathcal{O}_{T^{(m)}} \rightarrow \mathcal{O}_{U^{(m)}} \rightarrow \mathcal{O}_{T_{0}}$.

By Lemma $6.12, T_{0} \hookrightarrow T$ can be considered as an object in $\operatorname{CRIS}_{\text {Int }}^{(0)}\left(X^{(m)} / \tilde{S}\right)$. We define a functor

$$
\mathrm{G}^{\prime}:\left(\begin{array}{c}
\log 0 \text {-crystals } \\
\text { on } \operatorname{CRIS}_{\mathrm{Int}}^{(0)}\left(X^{(m)} / \tilde{S}\right)
\end{array}\right) \rightarrow\left(\begin{array}{c}
\log m \text {-crystals } \\
\text { on } \operatorname{CRIS}_{\text {Int }}^{(m)}(X / \tilde{S})
\end{array}\right)
$$

by $\mathrm{Gr}^{\prime}(\mathcal{E})_{(U, T, J . \delta)}:=\mathcal{E}_{\left(T_{0}, T, J+p \mathcal{O}_{T}, \delta\right)}$ for each $(U, T, J, \delta) \in \operatorname{CRIS}_{\text {Int }}^{(m)}(X / \tilde{S})$. We also obtain a functor

$$
\left(\begin{array}{c}
\log 0 \text {-crystals } \\
\text { on } \operatorname{CRIS}_{\text {Int }}^{(0)}\left(X^{(m)} / S\right)
\end{array}\right) \rightarrow\left(\begin{array}{c}
\log m \text {-crystals } \\
\text { on } \operatorname{CRIS}_{\text {Int }}^{(m)}(X / S)
\end{array}\right)
$$

in a similar manner and denote it also by $\mathrm{G}^{\prime}$ by abuse of notation.

Lemma 6.13. The following diagram of categories commutes

$$
\begin{gathered}
\left(\begin{array}{c}
\text { log } 0 \text {-crystals } \\
\text { on } \operatorname{CRIS}_{\text {Int }}^{(0)}\left(X^{(m)} / S\right)
\end{array}\right) \stackrel{\mathrm{G}^{\prime}}{\longrightarrow}\left(\begin{array}{c}
\text { log m-crystals } \\
\text { on } \operatorname{CRIS}_{\mathrm{Int}}^{(m)}(X / S)
\end{array}\right) \\
\downarrow \\
\left(\begin{array}{c}
\text { left } \mathcal{D}_{X^{(m)} / S^{-m o d u l e s}}^{(0)} \\
\text { on } X^{(m)}
\end{array}\right) \stackrel{F^{*}}{\longrightarrow}\left(\begin{array}{c}
\text { left } \mathcal{D}_{X / S^{(m)} \text {-modules }}^{\text {on } X}
\end{array}\right) .
\end{gathered}
$$

Here each of the vertical functors is defined by the composite of functors defined in Remark 3.5 and Remark 5.8.

Proof. Let $\mathcal{E}$ be a $\log 0$-crystal on $\operatorname{CRIS}_{\mathrm{Int}}^{(0)}\left(X^{(m)} / S\right)$. Let us consider the following diagram:

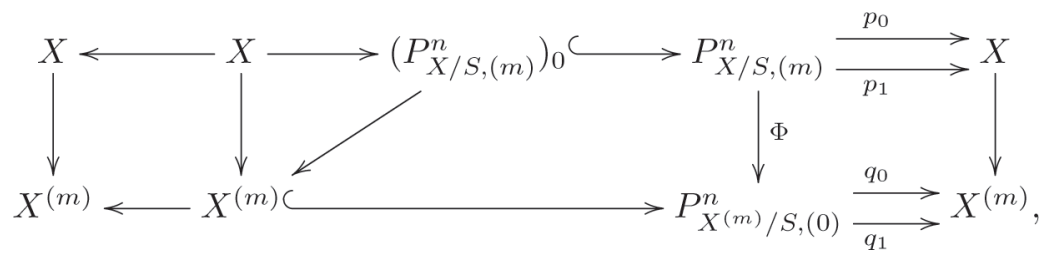


where the first, second and last vertical arrows are the $m$-th relative Frobenius morphisms, the slanting arrow is defined in Lemma 6.12, $\Phi$ is explained in Subsection 4.1.2 and $p_{0}, p_{1}$ (resp. $q_{0}, q_{1}$ ) are the first and the second projection. Then the two squares in the diagram are clearly commutative. The triangle in the diagram is commutative by construction of the map $\left(P_{X / S,(m)}^{n}\right)_{0} \rightarrow X^{(m)}$ (see Lemma 6.12). To prove the commutativity of the middle trapezoid, we may work locally. Then the assertion follows from the local description of $\Phi^{*}$ (see (2) of Proposition 4.5) and (1) of Proposition 3.2. Now, we see that $\Phi:\left(\left(P_{X / S .(m)}^{n}\right)_{0} \hookrightarrow P_{X / S .(m)}^{n}\right) \rightarrow\left(X^{(m)} \hookrightarrow P_{X^{(m)} / S,(0)}^{n}\right)$ is a morphism of $\log 0$-PD thickenings and, since $\mathcal{E}$ is a $\log 0$-crystal, we obtain the canonical isomorphisms

$$
\Phi^{*} \mathcal{E}_{\left(X^{(m)} \hookrightarrow P_{X(m) / S,(0)}^{n}\right)} \stackrel{\cong}{\cong} \mathcal{E}_{\left(\left(P_{X / S,(m)}^{n}\right)_{0} \hookrightarrow P_{X / S,(m)}^{n}\right)}=: \mathrm{G}^{\prime}(\mathcal{E})_{\left(X \hookrightarrow P_{X / S,(m)}^{n}\right)} .
$$

Therefore the $\log m$-PD stratification of $\mathrm{G}^{\prime}(\mathcal{E})$ is equal to that of $F^{*}\left(\mathcal{E}_{X}\right)$.

Finally, we are ready to prove Theorem 6.10 . By construction of the functor $\mathrm{G}_{\mathcal{I}_{X}^{g p}}$, Remark 4.24 and Remark 4.25 the following diagram of categories commutes:

$\left(\right.$ left $\mathcal{D}_{X^{(m)} / S}^{(0)}$-modules on $\left.X^{(m)}\right) \stackrel{F^{*}}{\longrightarrow}\left(\right.$ left $\mathcal{D}_{X / S}^{(m)}$-modules on $\left.X\right)$

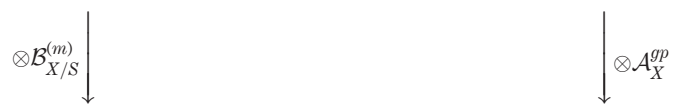

(left $\tilde{\mathcal{D}}_{X^{(m)} / S}^{(0)}$-modules on $\left.X^{(m)}\right) \stackrel{{ }^{\mathrm{G}_{I} g p}}{\longrightarrow}\left(\right.$ left $\tilde{\mathcal{D}}_{X / S}^{(m)}$-modules on $\left.X\right)$

So it suffices to show that $F^{*} \mathcal{K}_{\mathcal{X} / \mathcal{S}}^{(0)}$ is isomorphic to $\mathcal{K}_{\mathcal{X} / \mathcal{S}}^{(m)}$. Since the following diagram of categories commutes:

$$
\begin{aligned}
& \left(\begin{array}{c}
p \text {-torsion log 0-crystals } \\
\text { on } \operatorname{CRIS}_{\mathrm{Int}, f}^{(0)}\left(X^{(m)} / \tilde{S}\right)
\end{array}\right) \stackrel{\mathrm{G}^{\prime}}{\longrightarrow}\left(\begin{array}{c}
p \text {-torsion log } m \text {-crystals } \\
\text { on } \operatorname{CRIS}_{\mathrm{Int}, f}^{(m)}(X / \tilde{S})
\end{array}\right) \\
& \downarrow \text { A } \downarrow A^{\prime} \\
& \left(\begin{array}{c}
\log 0 \text {-crystals } \\
\text { on } \mathrm{CRIS}_{\mathrm{Int}}^{(0)}\left(X^{(m)} / S\right)
\end{array}\right) \stackrel{\mathrm{G}^{\prime}}{\longrightarrow}\left(\begin{array}{c}
\log m \text {-crystals } \\
\text { on } \mathrm{CRIS}_{\mathrm{Int}}^{(m)}(X / S)
\end{array}\right) \\
& \downarrow \\
& \left(\begin{array}{c}
\text { left } \mathcal{D}_{X^{(m)} / S^{(0)}}^{\text {-modules }} \\
\text { on } X^{(m)}
\end{array}\right) \stackrel{F^{*}}{\longrightarrow}\left(\begin{array}{c}
\text { left } \mathcal{D}_{X / S^{(m)}}^{(m) \text { modules }} \\
\text { on } X
\end{array}\right)
\end{aligned}
$$

where $A$ and $A^{\prime}$ are equivalences of categories in Lemma 5.16 and the right square is same as Lemma 6.13, we shall show that the functor $\mathrm{G}^{\prime}$ sends the $p$-torsion log 0crystal $\mathcal{K}_{\mathcal{X} / \mathcal{S}}^{(0)}$ to the $p$-torsion $\log m$-crystal $\mathcal{K}_{\mathcal{X} / \mathcal{S}}^{(m)}$. Let $(\tilde{U}, \tilde{T}, \tilde{J}, \tilde{\delta})$ be a $m$-PD 
thickening in $\operatorname{CRIS}_{\operatorname{Int}, f}^{(m)}(X / \tilde{S})$. Let $T$ denote the reduction of $\tilde{T}$ modulo $p$. Let us consider the following commutative diagram:

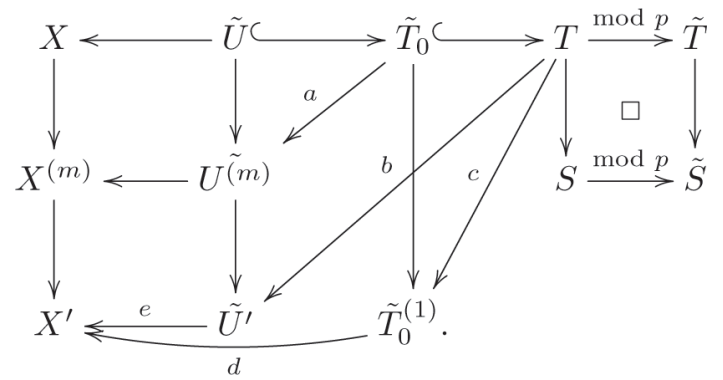

Here the slanting arrow $a$ is defined in Lemma 6.12, the slanting arrows $b$ and $c$ are defined in Lemma 5.11 and the arrow $d: \tilde{T}_{0}^{(1)} \rightarrow X^{\prime}=\left(X^{(m)}\right)^{(1)}$ is induced from the composition $\tilde{T}_{0} a U^{(m)} \rightarrow X^{(m)}$ via the functoriality of the first relative Frobenius morphism. Then, by definition, $\left(\mathrm{G}^{\prime}\left(\mathcal{L}_{\mathcal{X} / \mathcal{S}}^{(0)}\right)\right)_{(\tilde{U} \hookrightarrow \tilde{T})}=\left(\mathcal{L}_{\mathcal{X} / \mathcal{S}}^{(0)}\right)_{\left(\tilde{T}_{0} \hookrightarrow \tilde{T}\right)}$ is the étale sheaf of sets on $\tilde{T}$ of local liftings of the composition $d \circ c$. On the other hand, $\left(\mathcal{L}_{\mathcal{X} / \mathcal{S}}^{(m)}\right)_{(\tilde{U} \hookrightarrow \tilde{T})}$ is the étale sheaf of sets on $\tilde{T}$ of local liftings of the composition $f_{T / S}:=e \circ b$. Therefore the equality $\left(\mathrm{G}^{\prime}\left(\mathcal{L}_{\mathcal{X} / \mathcal{S}}^{(0)}\right)\right)_{(\tilde{U} \hookrightarrow \tilde{T})}=\left(\mathcal{L}_{\mathcal{X} / \mathcal{S}}^{(m)}\right)_{(\tilde{U} \hookrightarrow \tilde{T})}$ follows from the fact that the morphism $d \circ c$ is equal to the morphism $e \circ b$. Actually this is the identification as a torsor over $f_{T / S}^{*} \mathcal{T}_{X^{\prime} / S}$. So we have $\left(\mathrm{G}^{\prime}\left(\mathcal{E}_{\mathcal{X} / \mathcal{S}}^{(0)}\right)\right)_{(\tilde{U} \hookrightarrow \tilde{T})}=$ $\left(\mathcal{E}_{\mathcal{X} / \mathcal{S}}^{(m)}\right)_{(\tilde{U} \hookrightarrow \tilde{T})}$ and $\left(\mathcal{G}^{\prime}\left(\mathcal{K}_{\mathcal{X} / \mathcal{S}}^{(0)}\right)\right)_{(\tilde{U} \hookrightarrow \tilde{T})}=\left(\mathcal{K}_{\mathcal{X} / \mathcal{S}}^{(m)}\right)_{(\tilde{U} \hookrightarrow \tilde{T})}$. Consequently we obtain the equality $\mathrm{G}^{\prime}\left(\mathcal{K}_{\mathcal{X} / \mathcal{S}}^{(0)}\right)=\mathcal{K}_{\mathcal{X} / \mathcal{S}}^{(m)}$ as a $p$-torsion log $m$-crystal. This finishes the proof.

Acknowledgments. This paper is a revised version of the author's master thesis at the University of Tokyo. The author expresses his hearty thanks to Professor Atsushi Shiho for suggesting the topics, helpful discussions, carefully reading the draft of this paper, pointing out a lot of mistakes on it and valuable advice. Without his advice, this paper could not be realized. He also would like to thank the referee for reading this paper carefully, and for giving him many advices. This paper owes its existence to the work of Gros-Le Stum-Quirós [4], Lorenzon [7], Montagnon [9], Ogus-Vologodsky [10] and Schepler [11]. He thanks them heartily. This work was supported by the Program for Leading Graduate Schools, MEXT, Japan and Research Fellow of the Japan Society for the Promotion of Science.

\section{REFERENCES}

[1] Pierre Berthelot, Berthelot letter to Illusie, (1990).

[2] Pierre Berthelot, D-modules arithmétiques I. Opérateurs différentiels de niveau fini. Ann. Sci. École Norm. Sup. (4), 29(2) (1996), 185-272.

[3] Pierre Berthelot, D-module arithmétiques II. Descente par Frobenius, Mém. Soc. Math. France 81 (2000). 
[4] Michel Gros, Bernard Le Stum and Adolfo Quirós, A Simpson correspondance in positive characteristic, Publ. Res. Inst. Math. Sci. 46 (2010), 1-35.

[5] KaZUYA Kato, Logarithmic structures of Fontaine-Illusie, in Algebraic analysis, geometry, and number theory (Baltimore, MD, 1988), 191-224, Johns Hopkins Univ. Press, Baltimore, MD (1989).

[6] Bernard Le Stum and Adolfo Quirós, Transversal crystals of finite level. Ann. Inst. Fourier (Grenoble), 47(1) (1997), 69-100.

[7] Pierre Lorenzon, Indexed algebras associated to a log structure and a theorem of p-descent on log schemes, Manuscripta Mathematica 101 (2000), 271-299.

[8] KaZUAKI MiYatani, On the finitude of logarithmic crystalline cohomology of higher level, master thesis (2009).

[9] Claude Montagnon, Généralisation de la théorie arithmétique des D-modules à la géométrie logarithmique, Ph.D. thesis, L'université de Rennes I (2002); see http://tel.archives-ouvertes.fr/docs/00/04/52/24/PDF/tel-00002545.pdf.

[10] Arthur Ogus and Vladimir Vologodsky, Nonabelian Hodge theory in characteristic p. Publ. Math. Inst. Hautes Études Sci., 106 (2007), 1-138.

[11] Daniel SchePler, Logarithmic nonabelian Hodge theory in characteristic p, arXiv:0802.1977; see http://arxiv.org/pdf/0802.1977v1.pdf

[12] Carlos T. Simpson, Higgs bundles and local systems. Publ. Math. Inst. Hautes Études Sci., 75 (1992), 5-95.

Manoscritto pervenuto in redazione l'11 Aprile 2014. 
